\title{
GRAPH LAPLACE AND MARKOV OPERATORS ON A MEASURE SPACE
}

\author{
SERGEY BEZUGLYI AND PALLE E.T. JORGENSEN
}

\begin{abstract}
The main goal of this paper is to build a measurable analogue to the theory of weighted networks on infinite graphs. Our basic setting is an infinite $\sigma$-finite measure space $(V, \mathcal{B}, \mu)$ and a symmetric measure $\rho$ on $(V \times V, \mathcal{B} \times \mathcal{B})$ supported by a measurable symmetric subset $E \subset V \times V$. This applies to such diverse areas as optimization, graphons (limits of finite graphs), symbolic dynamics, measurable equivalence relations, to determinantal processes, to jumpprocesses; and it extends earlier studies of infinite graphs $G=(V, E)$ which are endowed with a symmetric weight function $c_{x y}$ defined on the set of edges $E$. As in the theory of weighted networks, we consider the Hilbert spaces $L^{2}(\mu), L^{2}(c \mu)$ and define two other Hilbert spaces, the dissipation space Diss and finite energy space $\mathcal{H}_{E}$. Our main results include a number of explicit spectral theoretic and potential theoretic theorems that apply to two realizations of Laplace operators, and the associated jump-diffusion semigroups, one in $L^{2}(\mu)$, and, the second, its counterpart in $\mathcal{H}_{E}$. We show in particular that it is the second setting (the energy-Hilbert space and the dissipation Hilbert space) which is needed in a detailed study of transient Markov processes.
\end{abstract}

\section{Contents}

1. Introduction

2. Basic setting

2.1. Discrete case: electrical networks

2.2. From discrete to measurable setting

2.3. Two basic models

3. Linear operators in functional spaces related to symmetric measures

3.1. Definitions of operators $R, \widetilde{R}, \Delta$, and $P$

3.2. A few facts about the operators $R, \widetilde{R}$

4. Markov processes associated with symmetric measures 30

4.1. Markov operators $\quad 30$

4.2. Harmonic functions for $P$

4.3. Markov processes

4.4. Reversible Markov processes 42

5. Dissipation space and stochastic analysis 433

6. Finite energy space: Decompositions and covariance computation 48

2010 Mathematics Subject Classification. 37B10, 37L30, 47L50, 60J45.

Key words and phrases. Laplace operator, standard measure space, symmetric measure, Markov operator, Markov process, harmonic function, dissipation space, finite energy space. 
6.1. $\mathcal{B}_{\text {fin }}$ as a connected graph 49

6.2. Definition and properties of the finite energy space $\mathcal{H}_{E}$

6.3. Energy space is embedded into dissipation space 53

6.4. Structure of the energy space 56

7. Spectral theory for graph Laplacians in $L^{2}(\mu) \quad 60$

8. Spectral theory of the graph Laplacian in the energy space 65

References 68

\section{INTRODUCTION}

Motivation. Recent works on graph Laplacians and Markov processes (details and definitions are given below) for networks suggest a duality between the two settings, (a) a discrete Laplacian $\Delta$, and (b) an associated Markov transition operator $P$. This duality approach is used in turn for answering questions in dynamics, such as deciding when a walk is transient or recurrent; identifying classes of harmonic functions, and an harmonic analysis; building path-space models, and formulate an associated theory of martingales and of boundary spaces.

As is known, this setting is as follows: (a) the graph Laplacians will have positive spectrum; and (b) the transition operators (generalized Perron-Frobenius operators) will be positive, in that they map positive functions to positive functions. However the setting of these studies is discrete; as is clear for example for graphs and networks. In other words, we have countable discrete sets of vertices and edges; and so the relevant Hilbert spaces will be defined from counting measures, weighted or not.

Nonetheless, there are many important applications where the framework of countable discrete sets of vertices $V$ and edges $E$ is much too restrictive. The list of applications is long, both connections to probability, analysis, signal processing and more: graphons (limits of finite graphs), determinantal processes, machine learning, jump processes, integral operators, harmonic analysis etc. Certainly there is a rich variety of Markov processes where the natural setting for state space is a general measure space. It is our purpose here, in the measure theoretic setting, to make precise the duality between the two, transition operator and "graph" Laplacian.

Of course for general measure spaces, the word "graph" should perhaps be given a different meaning; see below. Starting with a Markov transition operator, in the measure-dynamic setting, what is the dual Laplacian; and vice versa?

In the countable discrete cases from network models, spectral theory and the tools of dynamics rely on a certain Hilbert space that measures "energy" and dissipation, but there, one refers to weighted counting measures on the respective sets $V$ and $E$. Our present paper deals with measure theoretic dynamics. We answer the following three questions: (i) What are the relevant measures for the general setting; (ii) What are the correct notions of positivity for both operators in the measure theoretic setting; and (iii) What is then the extended duality between 
transition operator and Laplacian?

Discrete and measurable settings. We begin here with precise definitions, and clarifications of the three problems. We first point out explicit parallels between the main objects in the theory of discrete networks and their counterparts defined in the measurable framework. More details are given in Section 2.

In this paper, we focus on the study of a measurable analogue of countable weighted networks, which are known also by names electrical or resistance networks (we will use them as synonyms). We recall that $(G, c)$ is called a weighted network if $G=(V, E)$ is a countable connected locally finite graph with no loops, and $c=c_{x y}$ is a symmetric function defined on pairs of of connected vertices (a more detailed definition is given in Section 2). One can think of a countable network as a discrete measure space $(V, m)$ with the counting measure $m$. In general, the theory of weighted networks is built around two important operators acting on the space of functions $f: V \rightarrow \mathbb{R}$. They are the Laplace operator $\Delta$ and the Markov operator $P$.

$$
(\Delta f)(x):=\sum_{y \sim x} c_{x y}(f(x)-f(y)), \quad P(f)(x)=\sum_{y \sim x} p(x, y) f(y), \quad x \in V,
$$

where the transition probabilities are defined by

$$
p(x, y)=\frac{c_{x y}}{c(x)}, \quad c(x)=\sum_{y \sim x} c_{x y} .
$$

It is customary to study these operators in the Hilbert spaces naturally related to a network $(V, E, c)$ such as $l^{2}(V), l^{2}(V, c)$, and the finite energy Hilbert space $\mathcal{H}$. The Laplacian $\Delta$ generates the operator in the Hilbert spaces $l^{2}(V), l^{2}(V, c)$, and the finite energy space $\mathcal{H}$ which is formed by functions $f: V \rightarrow \mathbb{R}$ such that

$$
\|f\|_{\mathcal{H}}^{2}=\frac{1}{2} \sum_{x, y: x \sim y} c_{x y}(f(x)-f(y))^{2} .
$$

Our approach to the construction of a measurable analogue is based on the following setting. An infinite $\sigma$-finite measure space $(V, \mathcal{B}, \mu)$ is the underlying space ("vertices"), a symmetric subset $E \subset V \times V$ plays the role of "edges", and a symmetric measure $\rho$ supported by $E$ is an analogue of the function $c_{x y}$. Since $\rho$ is a measure in the product space $V \times V$, it can be disintegrated with respect to $\mu$, i.e.,

$$
\rho(f)=\int_{V} \rho_{x}(f) d \mu(x) .
$$

It is assumed that $\rho_{x}(V)=: c(x)$ is finite and locally integrable on $(V, \mathcal{B}, \mu)$. This property is analogous to local finiteness of discrete networks.

We define measurable analogues of the Laplacian and Markov operator from (1.1) as follows:

$$
\Delta(f)(x)=\int_{V}(f(x)-f(y)) d \rho_{x}(y), \quad P(f)(x)=\int_{V} f(y) d \rho_{x}(y),
$$


where $c(x) P(x, d y)=d \rho_{x}(y)$. A function $f$ satisfying the condition $\Delta f=0$ (or equivalently, $P f=f$ ) is called harmonic.

The corresponding Hilbert spaces are $L^{2}(V, \mathcal{B}, \mu), L^{2}(V, \mathcal{B}, \nu)$ where $d \nu(x)=$ $c(x) d \mu(x)$, and the finite energy space $\mathcal{H}_{E}$ with norm defined by

$$
\|f\|_{\mathcal{H}_{E}}^{2}=\iint_{V \times V}(f(x)-f(y))^{2} d \rho(x, y) .
$$

These definitions clarify the similarity between spaces and operators considered in discrete in measurable cases. More parallels can be found in Section 2, see Tables 1 and 2.

Main results and outline of the paper. The first part of the paper, Sections 2 and 3, contains principal definitions and notions that are constantly used in the paper. It is important to emphasize that we consider only infinite $\sigma$-finite measures on a standard Borel space $(V, \mathcal{B})$ because probability measures would correspond to finite networks. In Section 2 we recall several crucial results about the Laplacian and Markov operator in the context of weighted networks. The second part of Section 2 is mostly devoted to symmetric measures $\rho$ defined on symmetric Borel subsets $E$ of $V \times V$. These measures play the central role in our study. We give a few equivalent approaches to the definition of symmetric measures including polymorphisms and symmetric operators. Remark that this concept can be met in various areas of mathematics. One of them is the theory of graphons. At the end of this section we give two extreme models for symmetric measures: the first model is based on the case when $(\mu \times \mu)(E)>0$, and the second one deals with countable Borel equivalence relations $E$, i.e., $(\mu \times \mu)(E)=0$.

Section 3 contains the definitions and properties of the graph Laplace and Markov operators $\Delta$ and $P$, as well as of two auxiliary operators $R$ and $\widetilde{R}$. These operators, which are formally defined as integral operators on the space of Borel functions, can be realized as operators acting on Hilbert spaces, $L^{2}$-spaces and the finite energy space $\mathcal{H}_{E}$.

Section 4 deals with a Markov process generated by a Markov operator $P$. The difference from the classical approach to Markov processes is that we have to work with an infinite measure space. We focus here on the following topics: spectral properties of the operator $P$, harmonic functions for $P$, the Markov process generated by $P$, the path spaces $\Omega$ and $\Omega_{x}$, and the corresponding path measures, reversibility of the Markov process.

In Section 5, we define the dissipation space Diss whose analogue in discrete networks is used for the study of the finite energy space. The dissipation space Diss is, in fact, represented as an $L^{2}$-space with infinite measure. It turns out that the spaces we are interested in can be embedded into the dissipation space. This fact is extremely useful since it gives the possibility to apply the structure of the dissipation space to the study of our main objects considered now as operators in Diss. 
The finite energy space is thoroughly studied in Section 6. We first prove a curious result that can be interpreted as connectedness of a "graph" whose "vertices" are sets of finite measure in $(V, \mathcal{B}, \mu)$. To study the properties of $\mathcal{H}_{E}$, we utilize an isometric embedding of $\mathcal{H}_{E}$ into $L^{2}(\rho)$ and contractive embeddings of $\mathcal{H}_{E}$ into both $L^{2}(\nu)$ and Diss. A number of results about the norm of various elements of $\mathcal{H}_{E}$ is proved. We also characterize harmonic functions in the Hilbert space $\mathcal{H}_{E}$, and find conditions for orthogonality of elements of $\mathcal{H}_{E}$.

Sections 7 and 8 are devoted to the study of spectral properties of the Laplacians $\Delta_{2}$ and $\Delta_{\mathcal{H}}$, the Laplace operators acting in $L^{2}(\mu)$ and $\mathcal{H}_{E}$, respectively. It turns out that $\Delta_{2}$ is positive definite and self-adjoint unbounded operator in $L^{2}(\mu)$. On the other hand $\Delta_{\mathcal{H}}$ is a symmetric operator that admits many self-adjoint extensions.

Our main results can be found in Theorems 3.3, 4.4, 4.7, 4.9, 4.13, 6.9, 6.14, 7.5, 7.7, 8.5, Propositions 4.21, 5.7, and Corollaries 6.15, 6.17, 6.20,

\section{BASiC SETTING}

Our goal is to introduce and study the concepts which can be viewed as measurable analogues of basic objects from the weighted networks theory. For more details regarding discrete networks and their Laplacians, the reader may consult the following items [LP16, JS13, $\left.\mathrm{CGG}^{+} 16\right]$ and the papers cited there.

This section contains the main definitions of notions considered below. We first discuss the underlying measure space and symmetric measures. To illustrate parallels between discrete and measurable setting, we consider two models for a measurable setting.

2.1. Discrete case: electrical networks. For the reader's convenience, we briefly recall several principal facts and definitions from the theory of weighted networks.

Let $G=(V, E)$ denote a connected undirected locally finite graph with single edges between vertices such that the vertex set $V$ is (countably) infinite, and the edge set $E$ has no loops. The set $E(x):=\{y \in V: y \sim x\}$ of all neighbors of $x$ is finite for any vertex $x$. The edge $e \in E$ connecting vertices $x$ and $y$ is denoted by $(x y)$. The connectedness of $G$ means that, for any two vertices $x, y \in V$, there exists a finite path $\gamma=\left(x_{0}, x_{1}, \ldots, x_{n}\right)$ such that $x_{0}=x, x_{n}=y$ and $\left(x_{i} x_{i+1}\right) \in E$ for all $i$.

Definition 2.1. An weighted network $(G, c)$ is a weighted graph $G$ with a symmetric conductance function $c: V \times V \rightarrow[0, \infty)$, i.e., $c_{x y}=c_{y x}$ for any $(x y) \in E$. Moreover, $c_{x y}>0$ if and only if $(x y) \in E$. For any $x \in V$, the total conductance at $x$ is defined as

$$
c(x):=\sum_{y \sim x} c_{x y}
$$


Given a weighted network $(G, c)=(V, E, c)$ with fixed conductance function $c$, we associate the following three Hilbert spaces of functions on $V$ :

$$
\begin{gathered}
l^{2}(V):=\left\{u: V \in \mathbb{R}:\|u\|_{l^{2}}^{2}=\sum_{x \in V} u(x)^{2}<\infty\right\}, \\
l^{2}(V, c):=\left\{u: V \in \mathbb{R}:\|u\|_{l^{2}(V, c)}^{2}=\sum_{x \in V} c(x) u(x)^{2}<\infty\right\},
\end{gathered}
$$

and

$$
\begin{array}{r}
\mathcal{H}_{E}:=\text { equivalence classes of functions on } V \text { such } \\
\qquad\|u\|_{\mathcal{H}_{E}}^{2}=\frac{1}{2} \sum_{(x y) \in E} c_{x y}(u(x)-u(y))^{2}<\infty
\end{array}
$$

where $u_{1}$ and $u_{2}$ are equivalent if $u_{1}-u_{2}=$ constant. The Hilbert space $\mathcal{H}_{E}$ is called the finite energy space

We note that in this paper we focus on real-valued functions. The case of complex-valued functions is considered with obvious changes.

Definition 2.2. The Laplacian on $(G, c)$ is the linear operator $\Delta$ which is defined on the linear space of functions $f: V \rightarrow \mathbb{R}$ by the formula

$$
(\Delta f)(x):=\sum_{y \sim x} c_{x y}(f(x)-f(y)) .
$$

A function $f: V \rightarrow \mathbb{R}$ is called harmonic on $(G, c)$ if $\Delta f(x)=0$ for every $x \in V$.

The Laplace operator $\Delta$ can be realized as an operator either in $l^{2}(V)$, or in $l^{2}(V, c)$, or in the energy space $\mathcal{H}_{E}$. The corresponding operators, $\Delta_{2}, \Delta_{c}$, and $\Delta_{\mathcal{H}}$ are, in general, unbounded and densely defined. The description of their domains requires a careful study of dipoles, see details in [JP16, JP17]. We refer to the following literature devoted to the Laplace operator studied in the context of weighted graphs (electrical networks) theory: AJ12, AJSV13, JP14, JT15c, JT15b, JPT16, SS17.

To any conductance function $c$ on a network $G$, we associate a reversible Markov kernel $P=(p(x, y))_{x, y \in V}$ with transition probabilities defined by $p(x, y)=\frac{c_{x y}}{c(x)}$. Then, for any $x, y \in V$,

$$
p(x, y) c(x)=p(y, x) c(y)
$$

that is the Markov process defined by $P$ is reversible. Define the probability transition operator for $f: V \rightarrow \mathbb{R}$ by setting

$$
P(f)(x)=\sum_{y \sim x} p(x, y) f(y), \quad x \in V .
$$

Then $P$ is called the Markov operator. It is clear that the Laplace operator $\Delta$ can be represented in terms of the Markov operator as follows:

$$
\Delta(f)(x)=c(x)(f(x)-P(f)(x))
$$

or $\Delta=c(I-P)$ where $c$ stands for the operator of multiplication by $c$.

The operator $P$ defines also a Markov process $\left(P_{n}\right)$ on the probability path space $\left(\Omega_{x}, \mathbb{P}_{x}\right)$. Here $\Omega_{x}$ is the set of infinite paths beginning at $x \in V$, and $\mathbb{P}_{x}$ is the 
probability measure on $\Omega_{x}$ determined by transition probabilities $p(x, y)$. Let $X_{n}$ be the sequence of random variables on $\Omega_{x}$ such that $X_{n}(\omega)=\omega_{n}$.

A Markov process $\left(P_{n}\right)$ is called transient if the function

$$
G(x, y):=\sum_{n} p_{n}(x, y)
$$

is finite for any pair $x, y \in V\left(p_{n}(x, y)\right.$ denotes the probability of the event $\mathbb{P}_{x}\left(X_{n}=\right.$ $y)$ ).

We summarize the following results which can be found, in particular, in JP11, DJ11, JT15c, JP16].

Theorem 2.3. (1) $\Delta_{2}$ is essentially self-adjoint, generally unbounded operator with dense domain in $l^{2}(V)$;

(2) $\Delta_{\mathcal{H}}$ is an unbounded, non-negative, closed, and symmetric operator with dense domain in $\mathcal{H}_{E}$; in general, $\Delta_{\mathcal{H}}$ is not a self-adjoint operator;

(3) $P$ is bounded and self-adjoint in $l^{2}(V, c)$ and its spectrum is in $[-1,1]$.

In order to illustrate the parallels between discrete networks and measurable spaces, we give two tables below. They contain definitions of the main objects for countable weighted network and its continuous counterpart, the measurable space $(V \times V, \mathcal{B} \times \mathcal{B})$ equipped with a symmetric measure $\rho$. Table 1 is focused on the comparison of geometrical objects in the two cases such. On the other hand, Table 2 is about operators acting in the corresponding Hilbert spaces. More detailed definitions can be found in the text below.

Space and Network. In discrete models the set $V$ will typically be a specified set of vertices in a big network; generally countably infinite. In the non-discrete, or measurable, case, $V$ will instead be part of a measure space. In both cases, we will consider edges, and specified conductance functions. While the discrete case is better understood because its history, and a rich literature, in both pure and applied models, the continuous case (i.e., non-discrete) is perhaps less familiar. A common feature for the two is their use in the study of reversible Markov processes. While there is already a rich literature in the case of discrete networks (see cited references), the continuous, or rather, measurable, setting is of more recent vintage. It is the focus of our paper. However, a comparison between the two is useful, see Table 1. We will study infinite networks, both discrete and measurable, often as limits of finite ones. But many measure-space models arise in applications which do not make reference to limits of discrete counterparts.

Symmetry and Conductance. In the discrete models, symmetry refers to a function defined on the set $E$ of edges. In the special case of electrical networks of resisters, such a function could be a conductance; i.e., the reciprocal of resistance. There, functions on the set $V$ of vertices could be voltage, and functions on the edges current. Computations will then make use of Ohm's law, and Kirchhoof's law. Continuous or measurable models are more subtle; they may arise as limits of discrete ones, for example as graphons, but their study is interesting in its own right. 
Another instance of discrete vs continuous is classical potential theory: for example, a classical Laplacian is studied in numerical analysis as a limit of discretized Laplacians.

Laplacian, Markov operator and Transition probability. In the discrete setting, a typical case of interest is that of transition matrices for a Markov chain, for example in the study of dynamical systems described by Bratteli diagrams; and in the continuous case, it takes the form of a measurable family of transition probability measures, indexed by points $x$ in $V$, so that $P(x, \cdot)$ represents transition from $x$. Since our dynamical theories are based on a specified graph Laplacians, the corresponding Markov processes will be assumed reversible (defined in the paper).

Hilbert spaces. Our proofs will rely on the theory of operators in Hilbert space, and their corresponding spectral theory, but each of the operators under consideration entails its own Hilbert space. A given operator may be selfadjoint in one but not in another. As a result, we must introduce several weighed $l^{2}$ spaces (and $L^{2}$ spaces in the measure space case). Our study of boundary theory and of stochastic completeness entails the notion of energy Hilbert spaces, and dissipation Hilbert spaces, and each playing a crucial role in both the discrete and the continuous/measurable models.

In our outline above we briefly sketched and discussed some main themes, as they arise in both discrete settings, as well as in their measurable counterparts; the focus of our paper. We should stress that, especially for the discrete models, the existing literature is quite extensive. Below we cite a sample, but the reader will be able to supplement with papers cited there: BJ15b, BJ17, Cho14, DJ06, DJ11, Jor12, JP11, JP13, JP14, JP16, JP16, JP17, [JT15b, JT15c, LP16, SS17. Papers which cover aspects and applications in the measurable framework include [BJ17, GQ15, JKL02, JP12, JPT16, Kan08, Kec10, Luk16].

2.2. From discrete to measurable setting. We recall that, for every network $(V, E, c)$, an atomic measure space $(V, m)$ is given where $m$ is the counting measure. The conductance function $c$ defines another atomic measure $\rho$ on $E \subset V \times V$ by setting $\rho(x, y)=c_{x y}$. In what follows, we define, in terms of measure spaces, similar objects which can be regarded as analogues to the basic notions for weighted networks.

Measure space. Let $V$ be a separable completely metrizable topological space (a Polish space, for short), and let $\mathcal{B}$ be the $\sigma$-algebra of Borel subsets of $V$. Then $(V, \mathcal{B})$ is called a standard Borel space. We recall that all uncountable standard Borel spaces are Borel isomorphic, so that one can use any convenient realization of the space $V$. If $\mu$ is a continuous (i.e., non-atomic) Borel measure on $(V, \mathcal{B})$, then $(V, \mathcal{B}, \mu)$ is called a standard measure space. We use this name for both finite and $\sigma$-finite measure spaces. Also the same notation, $\mathcal{B}$ is applied for the $\sigma$-algebras of Borel sets and measurable sets of a standard measure space. In the context of measure spaces, we always assume that $\mathcal{B}$ is complete with respect to the measure $\mu$. By $\mathcal{F}(V, \mathcal{B})$ we denote the space of real-valued Borel functions on $(V, \mathcal{B})$. For 
TABLE 1. Comparison of discrete and continuous cases

\begin{tabular}{|c|c|c|}
\hline Objects & Discrete space & Measurable space \\
\hline Space & $\begin{array}{l}(V,|\cdot|) \text {, where } V \text { is vertices of a connected } \\
\text { graph } G \text { and }|\cdot| \text { is the counting measure }\end{array}$ & $\begin{array}{l}(V, \mathcal{B}, \mu) \text { standard } \sigma \text {-finite } \\
\text { measure space }\end{array}$ \\
\hline Network & $G=(V, E, c)$ weighted network & $(V \times V, \mathcal{B} \times \mathcal{B}, \rho)$ measure space \\
\hline Symmetry & $\begin{array}{c}c: E \rightarrow \mathbb{R} \text { conductance function } \\
\qquad c_{x y}=c_{y x}\end{array}$ & $\begin{array}{c}\rho=\int_{V} \rho_{x} d \mu(x) \text { symmetric measure } \\
\text { on a symmetric set } E \subset V \times V, \\
\rho(A \times B)=\rho(B \times A)\end{array}$ \\
\hline Conductance & $c(x)=\sum_{y \sim x} c_{x y}$ & $c(x)=\int_{V} d \rho_{x}=\rho_{x}(V)$ \\
\hline
\end{tabular}

$f \in \mathcal{F}(V, \mathcal{B})$ and a Borel measure $\mu$ on $(V, \mathcal{B})$, we write

$$
\mu(f)=\int_{V} f d \mu
$$

As a rule, we will deal only with continuous $\sigma$-finite measures on $(V, \mathcal{B})$ (unless the opposite is clearly indicated). This choice of measures is motivated by the discrete case where the counting measure plays the role of a $\sigma$-finite Borel measure on a measure space.

All objects, considered in the context of measure spaces (such as sets, functions, transformations, etc), are determined by modulo sets of zero measure (they are also called null sets). In most cases, we will implicitly use this mod 0 convention not mentioning the sets of zero measure explicitly.

Suppose now that a $\sigma$-finite continuous measure $\mu$ is chosen and fixed on $(V, \mathcal{B})$, so that $(V, \mathcal{B}, \mu)$ is a standard measure space. We denote by

$$
\mathcal{B}_{\text {fin }}=\mathcal{B}_{\text {fin }}(\mu)=\{A \in \mathcal{B}: \mu(A)<\infty\}
$$

the algebra of Borel sets of finite measure $\mu$. Clearly, the set $V$ can be partitioned into a disjoint countable union of sets $A_{i}$ from $\mathcal{B}_{\text {fin }}$. 
TABLE 2. Comparison of operators in discrete and continuous cases

\begin{tabular}{|c|c|c|}
\hline Objects & Discrete case & Measurable case \\
\hline Laplacian & $\Delta(f)(x)=\sum_{y \sim x} c_{x y}(f(x)-f(y))$ & $\Delta(f)(x)=\int_{V}(f(x)-f(y)) d \rho_{x}(y)$ \\
\hline $\begin{array}{l}\text { Markov } \\
\text { operator }\end{array}$ & $P(f)(x)=\frac{1}{c(x)} \sum_{y \sim x} c_{x y} f(y)$ & $P(f)(x)=\frac{1}{c(x)} \int_{V} f(y) d \rho_{x}(y)$ \\
\hline $\begin{array}{c}\text { Transition } \\
\text { probabilities }\end{array}$ & $p(x, y)=\frac{1}{c(x)} c_{x y}$ & $P(x, A)=\int_{V} \chi_{A}(y) \frac{1}{c(x)} d \rho_{x}(y)$ \\
\hline Hilbert spaces & $l^{2}(V), l^{2}(V, c)$ & $L^{2}(\mu), L^{2}(c \mu)$, Diss \\
\hline $\begin{array}{c}\text { Energy } \\
\text { space } \mathcal{H}_{E}\end{array}$ & $\begin{array}{c}\|f\|_{\mathcal{H}_{E}}^{2}= \\
\frac{1}{2} \sum_{x, y} c_{x y}(f(x)-f(y))^{2}\end{array}$ & $\begin{array}{c}\|f\|_{\mathcal{H}_{E}}^{2}= \\
\frac{1}{2} \int_{V \times V}(f(x)-f(y))^{2} d \rho(x, y)\end{array}$ \\
\hline $\begin{array}{l}\text { Finitely supported } \\
\text { functions }\end{array}$ & $\left\langle\delta_{x}, f\right\rangle_{\mathcal{H}_{E}}=\Delta f(x)$ & $\begin{array}{c}\left\langle\chi_{A}, f\right\rangle_{\mathcal{H}_{E}}=\int_{A} \Delta f d \mu \\
A \in \mathcal{B}_{\text {fin }}\end{array}$ \\
\hline
\end{tabular}

We notice that the set $\mathcal{B}_{\text {fin }}$ can be used to define a subset of Borel functions which is dense in every $L^{p}(\mu)$-space. For this, we take

$$
\mathcal{D}_{\text {fin }}:=\left\{\sum_{i \in I} a_{i} \chi_{A_{i}}: A_{i} \in \mathcal{B}_{\text {fin }}, a_{i} \in \mathbb{R},|I|<\infty\right\}=\operatorname{Span}\left\{\chi_{A}: A \in \mathcal{B}_{\text {fin }}\right\} .
$$

Symmetric measures. We first define the notion of a symmetric set.

Definition 2.4. Let $E$ be an uncountable Borel subset of the direct product $(V \times$ $V, \mathcal{B} \times \mathcal{B})$ such that:

(i) $(x, y) \in E \Longleftrightarrow(y, x) \in E$;

(ii) $E_{x}:=\{y \in V:(x, y) \in E\} \neq \emptyset, \quad \forall x \in X$;

(iii) for every $x \in V,\left(E_{x}, \mathcal{B}_{x}\right)$ is a standard Borel space where $\mathcal{B}_{x}$ is the the $\sigma$-algebra of Borel sets induced on $E_{x}$ from $(V, \mathcal{B})$. 
We call $E$ a symmetric set.

It follows from (iii) that the projection of $E$ on each of two margins is $V$.

We observe that conditions (ii) and (iii) are not related to the symmetry property; they are included in Definition 2.4 for convenience, so that we will not have to make additional assumptions.

The next definition of a symmetric measure is crucial for this paper.

Definition 2.5. Let $(V, \mathcal{B})$ be a standard Borel space. We say that a measure $\rho$ on $(V \times V, \mathcal{B} \times B)$ is symmetric if

$$
\rho(A \times B)=\rho(B \times A), \quad \forall A, B \in \mathcal{B} .
$$

If $\left(E_{x}, \mathcal{B}_{x}\right)$ is an uncountable standard Borel space for every $x \in V$, then the symmetric measure $\rho$ is called irreducible.

The meaning of the definition of irreducible symmetric measures is clarified in Section 4. Here we see that the projection of the support of the irreducible measure $\rho$ is the set $V$.

Lemma 2.6. If $\rho$ is a symmetric measure on $(V \times V, \mathcal{B} \times \mathcal{B})$, then the support of $\rho$, the set $E$, is mod 0 symmetric.

Proof. The proof is direct and easy, so that we leave it for the reader.

The following remark contains two natural properties of symmetric measures which are implicitly added to Definition 2.5.

Remark 2.7. (1) In the paper, we consider the symmetric measures whose supporting sets $E$ satisfy Definition 2.4. In other words, we require that, for every $x \in V$, the set $E_{x} \subset E$ is uncountable.

(2) In general the notion of a symmetric measure is defined in the context of standard Borel spaces $(V, \mathcal{B})$ and $(V \times V, \mathcal{B} \times \mathcal{B})$. But if a $\sigma$-finite measure $\mu$ is given on $(V, \mathcal{B})$, then we need to introduce a relation between $\rho$ and $\mu$. Let $\pi_{1}: V \times V \rightarrow V$ be the projection on the first coordinate. We require that the symmetric measure must satisfy the property $\rho \circ \pi_{1}^{-1} \ll \mu$. The meaning of this assumption is clarified in Theorem 2.8 below.

Assumption $\boldsymbol{A}$. Let $(V \times V, \mathcal{B} \times \mathcal{B})$ be a $\sigma$-finite measure space. In this paper we will assume that the symmetric measure $\rho$ is irreducible, i.e., it satisfies also the following properties: (i) $E_{x} \neq \emptyset$, and (ii) $\left(E_{x}, \rho_{x}\right)$ is a standard measure space for $\mu$-a.e. $x \in V$. That is the projection of $E$ onto $V$ is a measurable set of full measure $\mu$.

Measure disintegration. Every Borel set $E$ in the product space $V \times V$ can be partitioned into "vertical" (or "horizontal") fibers. These partitions give examples of the so called measurable partitions. The theory of measurable partitions, developed by Rohlin in [Roh49], is a useful tool for the study of measures on standard Borel spaces. The case of probability measures was studied, in general, in [Roh49]. It was proved that any probability measure admits a unique disintegration with respect to 
a measurable partition. For $\sigma$-finite measures, there are similar results establishing the existence of such a disintegration. We refer here to a theorem proved in [Sim12]. This theorem is formulated below in the form which is adapted to our purposes.

Denote by $\pi_{1}$ and $\pi_{2}$ the projections from $V \times V$ onto the first and second factor, respectively. Then $\left\{\pi_{1}^{-1}(x): x \in V\right\}$ and $\left\{\pi_{2}^{-1}(y): y \in V\right\}$ are the measurable partitions of $V \times V$ into vertical and horizontal fibers.

Theorem 2.8 ([Sim12]). For a $\sigma$-finite measure space $(V, \mathcal{B}, \mu)$, let $\rho$ be a $\sigma$-finite measure on $(V \times V, \mathcal{B} \times \mathcal{B})$ such that $\rho \circ \pi_{1}^{-1} \ll \mu$. Then there exists a unique system of conditional $\sigma$-finite measures $\left(\widetilde{\rho}_{x}\right)$ such that

$$
\rho(f)=\int_{V} \widetilde{\rho}_{x}(f) d \mu(x), \quad f \in \mathcal{F}(V \times V, \mathcal{B} \times \mathcal{B}) .
$$

We apply Theorem 2.8 to a symmetric $\sigma$-finite measure $\rho$ with support $E$. Here $E=\operatorname{supp}(\rho)$ denotes a subset of $V \times V$ such that $\rho((V \times V) \backslash E)=0$. Clearly, this set is defined up to a set of zero measure.

Let $E$ be partitioned into the fibers $\{x\} \times E_{x}$. Then, by Theorem 2.8, there exists a unique system of conditional measures $\widetilde{\rho}_{x}$ such that, for any $\rho$-integrable function $f(x, y)$, we have

$$
\iint_{V \times V} f(x, y) d \rho(x, y)=\int_{V} \widetilde{\rho}_{x}(f) d \mu(x) .
$$

It is obvious that, for $\mu$-a.e. $x \in V, \operatorname{supp}\left(\widetilde{\rho}_{x}\right)=\{x\} \times E_{x}$ (up to a set of zero measure).

In the following remark we collect several facts that clarify the essence of the defined objects.

Remark 2.9. (1) We first remark note that formulas involving integrals (see, e.g., (2.10) and (2.12) ) are understood in the sense of the extended real line, i.e., the infinite value of measurable functions are allowed.

(2) We would like to clarify our notation. It follows from Theorem 2.8 that we have a measurable field of sets $x \mapsto E_{x} \subset V$ and a measurable field of $\sigma$-finite Borel measures $x \mapsto \rho_{x}$ on $(V, \mathcal{B})$ where the measures $\rho_{x}$ are defined by the relation

$$
\widetilde{\rho}_{x}=\delta_{x} \times \rho_{x} .
$$

Hence, relation (2.10) can be also written as

$$
\iint_{V \times V} f(x, y) d \rho(x, y)=\int_{V}\left(\int_{V} f(x, y) d \rho_{x}(y)\right) d \mu(x) .
$$

In other words, we have a measurable family of measures $\left(x \mapsto \rho_{x}\right)$ (which is called a random measure), and it defines a new measure $\nu$ on $(V, \mathcal{B})$ by setting

$$
\nu(A):=\int_{V} \rho_{x}(A) d \mu(x), \quad A \in \mathcal{B}
$$

In contrast to the definition of $\rho$, we consider the measure $\rho_{x}$ to be defined on the subset $E_{x}$ of $(V, \mathcal{B}), x \in V$. 
(3) The symmetry of the set $E$ allows us to define a "mirror" image of the measure $\rho$. Let $E^{y}:=\{x \in V:(x, y) \in E\}$, and let $\left(\widetilde{\rho}^{y}\right)$ be the system of conditional measures with respect to the partition of $E$ into the sets $E^{y} \times\{y\}$. It can be easily proved, using the symmetry of $\rho$, that, for the measure,

$$
\rho^{\prime}=\int_{V} \widetilde{\rho}^{y} d \mu(y)
$$

the relation $\rho=\rho^{\prime}$ holds.

(4) It is worth noting that, in general, the set $E$, the support of a symmetric measure $\rho$, do not need to be a set of positive measure with respect to $\mu \times \mu$. In other words, we admit both the cases: (a) $\rho$ is equivalent to the product measure $\mu \times \mu$, (b) $\rho$ and $\mu \times \mu$ are mutually singular.

(5) To simplify our notation, we will always write $\int_{V} f d \rho_{x}$ and $\iint_{V \times V} f d \rho$ though the measures $\rho_{x}$ and $\rho$ have the supports $E_{x}$ and $E$, respectively.

Assumption $\boldsymbol{B}$. In general, when a $\sigma$-finite measure $\rho$ is disintegrated, the measures $\widetilde{\rho}_{x}$ supported by fibers $\{x\} \times E_{x}, x \in V$ are also $\sigma$-finite. In this paper, we will consider the class of symmetric measures for which

$$
0<c(x):=\rho_{x}(V)<\infty
$$

for $\mu$-a.e. $x \in V$. This assumption is made in accordance with local finiteness of weighted networks, see (2.1).

As an immediate consequence of Remark 2.9 (3), we have the following important formula.

Lemma 2.10. For a symmetric measure $\rho$,

$$
\iint_{V \times V} f(x, y) d \rho(x, y)=\iint_{V \times V} f(y, x) d \rho(x, y)
$$

or

$$
\int_{V} \int_{V} f(x, y) d \rho_{x}(y) d \mu(x)=\int_{V} \int_{V} f(x, y) d \rho_{y}(x) d \mu(y) .
$$

In particular, relation (2.15) is used to prove the equivalence of the measures $\mu$ and $\nu$.

Lemma 2.11. Suppose that $c(x)=\rho_{x}(V)$ is as in (2.14) for $\mu$-a.e. $x \in V$. The measure $\nu$ defined in (2.13) is equivalent to $\mu$, and $d \nu(x)=c(x) d \mu(x)$. 
Proof. For any set $A \in \mathcal{B}$, we obtain from (2.13) and (2.15) that

$$
\begin{aligned}
\nu(A) & =\int_{V} \rho_{x}(A) d \mu(x) \\
& =\int_{V} \int_{V} \chi_{A}(y) d \rho(x, y) \\
& =\int_{V} \int_{V} \chi_{A}(x) d \rho(x, y) \\
& =\int_{V} \chi_{A}(x) c(x) d \mu(x) \\
& =\int_{A} c(x) d \mu(x) .
\end{aligned}
$$

Hence, $\nu$ is equivalent to $\mu$ and $c(x)$ is the Radon-Nikodym derivative.

Symmetric measures vs symmetric operators. Symmetric measures $\rho$ on $(V \times V, \mathcal{B} \times \mathcal{B})$ can be described in terms of positive linear operators $R$ acting in appropriate functional spaces.

For a given Borel measure measure $\rho=\left(\rho_{x}\right)$ on the space $(V \times V, \mathcal{B} \times \mathcal{B})$, we can define a linear operator by setting

$$
R(f)(x):=\int_{V} f(y) d \rho_{x}(y)=\rho_{x}(f), \quad f \in \mathcal{F}(V, \mathcal{B}) .
$$

Clearly, $R$ is a positive operator, i.e., $f \geq 0 \Longrightarrow R(f) \geq 0$.

Remark 2.12. (1) In this paper we will consider a number of linear operators acting in some functional spaces. We define them formally as operators on the space of Borel functions $\mathcal{F}(V, \mathcal{B})$. But our main interest is focused on their realizations as operators acting in $L^{p}(V, \mathcal{B}, \mu)$-spaces $p=1,2$ and some other Hilbert spaces (see next sections). In particular, we discuss the properties of $R$ (and a more general operator $\widetilde{R}$ ) in Section 3 .

(2) We recall that a Borel measure can be determined as a positive functional on a space of functions. In particular, a measure is completely defined by its values on a dense subset of functions. In the case of a measure $\rho$ on $V \times V$, it suffices to determine $\rho$ on the so-called "cylinder functions" $(f \otimes g)(x, y):=f(x) g(y)$ (this approach corresponds to the definition of a measure on rectangles first).

Denote by $\mathbb{1}$ the constant function on $(V, \mathcal{B}, \mu)$ which equals 1 at every point $x$.

Proposition 2.13. Let $(V, \mathcal{B}, \mu)$ be a $\sigma$-finite standard measure space. Let $\rho=$ $\int_{V} \delta_{x} \times \rho_{x} d \mu(x)$ be a continuous Borel measure on $(V \times V, \mathcal{B} \times B)$. The following are equivalent:

(1) $\rho$ is a symmetric measure such that

$$
0<c(x)=\rho_{x}(V)<\infty
$$

for $\mu$-a.e. $x \in V$. 
(2) There exists a positive operator $R$ in $\mathcal{F}(V, \mathcal{B})$ such that $R(\mathbb{1})(x)=c(x)$ and

$$
\int_{V} f R(g) d \mu=\int_{V} R(f) g d \mu
$$

for any $f, g \in F(X, \mathcal{B})$.

The operator $R$ satisfying (2.18) is called symmetric.

Proof. $(1) \Rightarrow(2)$. This implication is straightforward: if $\rho$ is a symmetric measure, define a positive operator $R$ as in (2.17). Then, using the disintegration of $\rho$, we have

$$
\begin{aligned}
\rho(A \times B) & =\iint_{V \times V} \chi_{A}(x) \chi_{B}(y) d \rho(x, y) \\
& =\int_{V} \chi_{A}(x) R\left(\chi_{B}\right)(x) d \mu(x) .
\end{aligned}
$$

Similarly,

$$
\rho(B \times A)=\int_{V} \chi_{B}(x) R\left(\chi_{A}\right)(x) d \mu(x) .
$$

Since $\rho(A \times B)=\rho(B \times A)$, we obtain that (2.18) holds for any simple function, and therefore the result follows.

$(2) \Rightarrow(1)$. The above proof can be used to show that the converse implication also holds.

Polymorphisms and symmetric measures. The approach to symmetric measures, which uses a positive operator $R$ (see Proposition 2.13), can be developed in more general setting. The key concept here is the notion of a polymorphism which was defined and studied in a series of papers by A. Vershik. It turns out that the main objects of ergodic theory can be considered in the framework of polymorphisms. We refer to [Ver00, Ver05] for further details.

Let $\mu_{1}$ and $\mu_{2}$ be Borel measures on a standard Borel space $(X, \mathcal{B})$. By definition, a polymorphism $\Pi$ of a standard Borel space $(X, \mathcal{B})$ to itself is a diagram consisting of an ordered triple of standard measure spaces:

$$
\left(X, \mathcal{B}, \mu_{1}\right) \stackrel{\pi_{1}}{\longleftarrow}(X \times X, \mathcal{B} \times \mathcal{B}, m) \stackrel{\pi_{2}}{\longrightarrow}\left(X, \mathcal{B}, \mu_{2}\right),
$$

where $\pi_{1}$ and $\pi_{2}$ are the projections onto the first and second component of the product space $(X \times X, \mathcal{B} \times \mathcal{B}, m)$, and $m$ is a measure on $(X \times X, \mathcal{B} \times \mathcal{B})$ such that $m \circ \pi_{i}^{-1}=\mu_{i}, i=1,2$. Remark that this notion is also used in the theory of optimal transport, see e.g. [Vil09].

This definition can be naturally extended to the case of two distinct measure spaces $\left(X_{i}, \mathcal{B}_{i}, \mu_{i}\right), i=1,2$. Then, the above definition gives a polymorphism defined between these measure spaces.

Suppose that $R$ is a positive operator acting on $\mathcal{F}(V, \mathcal{B})$. Then $R$ defines an action on the space of measures: if $\mu$ is a Borel measure on $(V, \mathcal{B})$, then one defines

$$
\mu R(f):=\int_{V} R(f) d \mu \text {. }
$$


More details can be found, fore example, in [BJ17.

Definition 2.14. Let $(V, \mathcal{B}, \mu)$ be a measure space, and let $R$ be a positive operator defined on Borel functions $\mathcal{F}(V, \mathcal{B})$ such that

$$
\int_{V} f(x) R(g)(x) d \mu(x)=\int_{V} R(f)(x) g(x) d \mu(x)
$$

for any functions $f, g \in \mathcal{F}(V, \mathcal{B})$.

Then the polymorphism

$$
\mathfrak{R}:=(V, \mathcal{B}, \mu R) \stackrel{\pi_{1}}{\longleftarrow}(V \times V, \mathcal{B} \times \mathcal{B}, \rho) \stackrel{\pi_{2}}{\longrightarrow}(V, \mathcal{B}, \mu R),
$$

is called a symmetric polymorphism defined by a positive operator $R$ and measure $\mu$ (here $\mu R$ is defined by (2.14)).

The following result relates the notions of symmetric measures and symmetric polymorphisms.

Proposition 2.15. Suppose that a symmetric measure $\rho$ on $(V \times V, \mathcal{B} \times \mathcal{B})$ satisfies the property

$$
c(x)=\rho_{x}(\mathbb{1}) \in(0,+\infty) \text { for } \mu \text {-a.e. } x \in V .
$$

Then $\rho$ defines a positive symmetric operator $R$ and a polymorphism

$$
\mathfrak{R}:=(V, \mathcal{B}, \nu) \stackrel{\pi_{1}}{\longleftarrow}(V \times V, \mathcal{B} \times \mathcal{B}, \rho) \stackrel{\pi_{2}}{\longrightarrow}(V, \mathcal{B}, \nu)
$$

such that $\nu=\mu R$ and

$$
\rho(f \otimes g)=\int_{V} f R(g) d \mu(x) .
$$

Conversely, suppose that a positive operator $R$ is defined on Borel functions over $(V, \mathcal{B}, \mu)$ and $R(\mathbb{1})=c(x)$. Then relation (2.20) defines a measure $\rho$ on $(V \times V, \mathcal{B} \times$ $\mathcal{B})$. The measure $\rho$ is symmetric if and only if, for any functions $f$ and $g$,

$$
\int_{V} f R(g) d \mu(x)=\int_{V} R(f) g d \mu(x) .
$$

Proof. These results have been partially proved in Proposition 2.13. The statements involving the notion of a polymorphism follow directly from the definitions. We leave the details to the reader.

Question A. Let $\rho$ be a symmetric measure on $(V \times V, \mathcal{B} \times \mathcal{B})$. Denote by $\mathcal{P}(\rho)$ the set of all pairs $(R, \mu)$, where $R$ is a positive symmetric operator on $\mathcal{F}(V, \mathcal{B})$ and $\mu$ is a measure on $(V, \mathcal{B})$, such that relation $(2.20)$ holds. Proposition 2.15 states that the set $\mathcal{P}(\rho)$ is not empty (provided finiteness of $c(x)$ ). Suppose that $(R, \mu)$ and $\left(R^{\prime}, \mu^{\prime}\right)$ are two pairs from the set $\mathcal{P}(\rho)$, i.e., they define the same $\rho$. What relations hold between $(R, \mu)$ and $\left(R^{\prime}, \mu^{\prime}\right)$ ? As was shown in Proposition 2.15, a pair $(R, \mu)$ must satisfy the conditions: $\mu R=c \mu$ where $c(x)=R(\mathbb{1})(x)$; and $\rho \circ \pi_{1}^{-1}=\rho \circ \pi_{2}^{-1}=c \mu$. 
Example 2.16 (Applications). We give here a few obvious examples of symmetric measures $\rho$ (more details can be found in Subsection 2.3).

(1) Let the measure $\nu$ be defined on $(V, \mathcal{B}, \mu))$ by $d \nu(x)=c(x) d \mu(x)$. Set $\rho_{0}(A \times$ $B):=\nu(A \cap B)$ where $A, B \in \mathcal{B}$. Then $\rho_{0}$ is a symmetric measure on $(V \times V, \mathcal{B} \times \mathcal{B})$ such that $R_{0}(\mathbb{1})=c(x)$, where $R_{0}$ is the corresponding symmetric operator whose action on functions is given by $R_{0}(f)=c(x) f(x)$.

(2) Let $c_{x y}$ be a symmetric function defined on a symmetric set $E \subset V \times V$. Consider a measurable field of finite Borel measures $x \mapsto \rho_{x}^{c}$ where $\rho_{x}^{c}$ is supported by $c_{x y}$. Then, setting

$$
\rho^{c}=\int_{V} c_{x y} d \rho_{x}^{c}(y)
$$

we define a symmetric measure $\rho^{c}$ on the set $E$.

(3) Our approach in the study of symmetric measures, and the corresponding graph Laplace operators, is close to the basic setting of the theory of graphons and graphon operators. We refer to several basic works in this theory $\mathrm{BCL}^{+} 08$, $\mathrm{BCL}^{+} 12$, Lov12, Jan13, APSS17. More references can be found in Lov12, Jan13. Informally speaking, a graphon is the limit of a converging sequence of finite graphs with increasing number of vertices. Formally, a graphon is a symmetric measurable function $W:(\mathcal{X}, m) \times(\mathcal{X}, m) \rightarrow[0,1]$ where $(\mathcal{X}, m)$ is a probability measure space. The linear operator $\mathbb{W}: L^{2}(\mathcal{X}, m) \rightarrow L^{2}(\mathcal{X}, m)$ acting by the formula

$$
\mathbb{W}(f)(x)=\int_{\mathcal{X}} W(x, y) f(y) d m(y)
$$

is called the graphon operator. The properties of $\mathbb{W}$ are studied in [APSS17.

Below in Section 3, we consider a similar operator $\widetilde{R}$ defined by a symmetric measure $\rho$. The principal difference is that we consider infinite measure spaces and symmetric functions which are not bounded, in general.

(4) Another application of our results can be used in the theory of determinantal measures and determinantal point processes, see e.g. LLyo03, HKPV09, BQ15, BO17]. For example, the result of [Gho15, Proposition 4.1] gives the formula for the norm in the energy space for a specifically chosen symmetric measure $\rho$. To make this statement more precise, we quote loosely the proposition proved in [Gho15:

Let $\Pi$ be a determinantal point process on a locally compact space $(X, \mu)$ with positive definite determinantal kernel $K(\cdot, \cdot)$ such that $K$ is an integral operator on $L^{2}(\mu)$. Then, for every compactly supported function $\psi$,

$$
\operatorname{Var}\left[\int_{x} \psi d[\Pi]\right]=\iint_{X \times X}|\psi(x)-\psi(y)|^{2}|K(x, y)|^{2} d \mu(x) d \mu(y) .
$$

This formula is exactly the formula for the norm in the energy space when the symmetric measure $\rho$ is defined by the symmetric function $K(x, y): d \rho(x, y)=$ $|K(x, y)|^{2} d \mu(x) d \mu(y)$, see Section 6 below.

(5) Another interesting application of symmetric measures and finite energy space is related to Dirichlet forms, see e.g., [MR92, MR95. We mention here the BeurlngDeny formula as given in [MR92]. It states that a symmetric Dirichlet form on 
$L^{2}(U)$, where $U$ is an open subset in $\mathbb{R}^{d}$, can be uniquely expressed as follows:

$$
\begin{aligned}
\mathcal{E}(u, v)= & \sum_{i, i=1}^{d} \int \frac{\partial u}{\partial x_{i}} \frac{\partial v}{\partial x_{j}} d \nu_{i j} \\
& +\int_{(U \times U) \backslash \operatorname{diag}}(u(x)-u(y))(v(x)-v(y)) J(d x, d y) \\
& +\int u v d k
\end{aligned}
$$

Here $u, v \in C_{0}^{\infty}(U), k$ is a positive Radon measure on $U \subset \mathbb{R}^{d}$, and $J$ is a symmetric measure on $(U \times U) \backslash$ diag. The first term on the right hand side in this formula is called the diffusion term, the second, the jump term, and the last, the killing term; a terminology deriving from their use in the study of general Levy processes App09. We see that the second term in this formula corresponds to the inner product in the finite energy space $\mathcal{H}_{E}$ (details are in Section 6 below).

2.3. Two basic models. We consider here two models which illustrate the setting described in Subsection 2.2. The first model is based on the case when the support of a symmetric measure $\rho$ is of positive measure $\mu \times \mu$. The other model deals with a countable Borel equivalence relation $\mathcal{E}$ which supports a singular measure $\rho$ with respect to $\mu \times \mu$.

$1^{\text {st }}$ case: $(\mu \times \mu)(E)>0$. Suppose that $(x, y) \mapsto c_{x y}$ is a positive real-valued Borel function whose domain is a symmetric Borel set $E \subset V \times V$ of positive measure $\mu \times \mu$. Additionally, we require that $c_{x y}$ is symmetric, i.e.,

$$
c_{x y}=c_{y x}, \quad \forall(x, y) \in E .
$$

By analogue with the theory of electrical networks, the function $c_{x y}$ is called a conductance function.

Without loss of generality, we can assume that the function $(x, y) \mapsto c_{x y}$ is defined everywhere on $V \times V$ by setting $c_{x y}=0$ for $(x, y) \notin E$.

Assumption $C$ : (1) For $\mu$-a.e. $x \in V$, the function $c_{x}(\cdot)=c_{x}$, is $\mu$-integrable, i.e.,

$$
c(x):=\int_{V} c_{x y} d \mu(y)
$$

is positive and finite for $\mu$-a.e. $\mathrm{x} \in V$.

(2) We also assume that $c(x) \in L_{\mathrm{loc}}^{1}(\mu)$, i.e., for any $A \in \mathcal{B}_{\text {fin }}$,

$$
\int_{A} c(x) d \mu(x)<\infty .
$$

These assumptions hold automatically for the discrete case of electrical networks. Note that the origin of condition (2) lies in local finiteness of graphs used in networks. 
Next, we define (in terms of $\mu$ and $c_{x y}$ ) two measures, $\nu$ and $\rho$, on $V$ and $E$, respectively.

Definition 2.17. Let $\rho$ be a $\sigma$-finite Borel measure on $E$ such that the RadonNikodym derivative of $\rho$ with respect to $\mu \times \mu$ is $c_{x y}$, i.e.,

$$
\frac{d \rho}{d(\mu \times \mu)}(x, y)=c_{x y}, \quad(x, y) \in E .
$$

For every $x \in V$, we define a measure $\rho_{x}$ on $(V, \mathcal{B})$ by the formula

$$
\rho_{x}(A)=\int_{A} c_{x y} d \mu(y), \quad A \in \mathcal{B} .
$$

In other words, $d \rho_{x}(y)=c_{x y} d \mu(y)$.

Clearly, the measures $\rho$ and $\rho_{x}$ are uniquely determined by $\mu$ and $c_{x y}$.

In the following assertion, we collect the properties of measures $\rho$ and $\rho_{x}$ that follow from the definition.

Lemma 2.18. Suppose that $(V, \mathcal{B}, \mu)$ is a standard measure space and $c_{x y}$ is a symmetric function on $E \subset V \times V$ as above. Then:

(1) The supports of $\rho$ and $\rho_{x}, x \in V$, are the sets $E \subset V \times V$, and $E_{x} \subset V$, respectively.

(2) The measure $\rho$ can be disintegrated with respect to the "vertical" and "horizontal" partitions $E=\bigcup_{x \in V}\{x\} \times E_{x}$ and $E=\bigcup_{y \in V} E^{y} \times\{y\}$ such that

$$
\rho=\int_{E} \delta_{x} \times \rho_{x} d \mu(x)=\int_{E} \rho^{y} \times \delta_{y} d \mu(y) .
$$

(3) The measure $\rho$ is symmetric:

$$
\rho(A \times B)=\rho(B \times A), \quad A, B \in \mathcal{B},
$$

or equivalently,

$$
\int_{E} f(x, y) d \rho(x, y)=\int_{E} f(y, x) d \rho(x, y)
$$

where $f$ is any Borel function on $(V \times V, \mathcal{B} \times \mathcal{B})$.

(4) For $\mu$-a.e. $x \in V$,

$$
\rho_{x}(V)=\rho_{x}\left(E_{x}\right)=c(x) .
$$

(5) The family of measures $\left(\rho_{x}\right)$ determines a positive linear operator $R$

$$
R(f)(x):=\int_{V} f(y) d \rho_{x}(y)=\int_{V} c_{x y} f(y) d \mu(y) .
$$

acting on $\mathcal{F}(X, \mathcal{B})$ such that, for any Borel functions $f$ and $g$,

$$
\rho(f \otimes g)=\int_{V} f(x) R(g)(x) d \mu(x)=\int_{V} R(f)(x) g(x) d \mu(x) .
$$


Remark 2.19. As stated in Lemma 2.18, the measures $\rho$ and $\rho_{x}$ are supported by the sets $E$ and $E_{x}$ where the functions $c_{x y}$ and $c_{x}: y \rightarrow c_{x y}$ are positive. Hence, we could equally use the formulas

$$
\iint_{V \times V} c_{x y} f(x, y) d \mu(x) d \mu(y)=\int_{E} f(x, y) d \rho(x, y)
$$

and

$$
\int_{V} c_{x y} f(y) d \mu(y)=\int_{E_{x}} f(y) d \rho_{x}(y) .
$$

Proof of Lemma 2.18. The first assertion is obvious due to the definition of the conductance function $c_{x y}$.

To see that (2) holds, we compute for arbitrary functions $f$ and $g$ :

$$
\begin{aligned}
\rho(f(x) \otimes g(y)) & =\int_{E} c_{x y} f(x) g(y) d \mu(y) d \mu(x) \\
& =\int_{V} f(x) \rho_{x}(g) d \mu(x) \\
& =\int_{V}\left(\delta_{x} \times \rho_{x}\right)(f \otimes g) d \mu(x) .
\end{aligned}
$$

Similarly, one can show that

$$
\rho(f(x) \otimes g(y))=\int_{V}\left(\rho^{y} \times \delta_{y}\right)(f \otimes g) d \mu(y) .
$$

To finish the proof, we note that the space spanned by cylinder functions is dense in $L^{1}(\rho)$.

For (3), we find that

$$
\begin{aligned}
\rho(A \times B) & =\int_{E} c_{x y} \chi_{A}(x) \chi_{B}(y) d \mu(y) d \mu(x) \\
& =\int_{V} \chi_{A}(x) \rho_{x}\left(\chi_{B}\right) d \mu(x) .
\end{aligned}
$$

On the other hand, since $c_{x y}=c_{y x}$, we have

$$
\begin{aligned}
\rho(B \times A) & =\int_{E} c_{y x} \chi_{A}(y) \chi_{B}(x) d \mu(x) d \mu(y) \\
& =\int_{V} \chi_{A}(y) \rho_{y}\left(\chi_{B}\right) d \mu(y),
\end{aligned}
$$

and the result follows.

Statement (4) of the lemma follows from the disintegration formula used in (2), the assumption about $c(x)$, and from Definition 2.17 . 
For (5), we obtain

$$
\begin{aligned}
\rho(f \otimes g) & =\int_{E} f(x) g(y) d \rho(x, y) \\
& =\int_{V} f(x)\left(\int_{V} g(y) d \rho_{x}(y)\right) d \mu(x) \\
& =\int_{V} f R(g) d \mu .
\end{aligned}
$$

Clearly, if $f \geq 0$, then $R(f) \geq 0$, i.e., $R$ is a positive operator.

More generally, we can treat $x \mapsto \rho_{x}$ as a measurable field of measures defined on $(V, \mathcal{B})$. We see that relation (2.23) and (2.24) define such a field. This means that, for any $A \in \mathcal{B}$, the function $x \mapsto \rho_{x}(A)$ is measurable on $(V, \mathcal{B}, \mu)$. In other words, this field of measures $x \mapsto \rho_{x}$ is called a random measure [Kal83, Aar97].

It follows from this observation that we can define a new measure $\nu$ on $(V, \mathcal{B})$ by setting

or

$$
\nu(A)=\int_{V} \rho_{x}(A) d \mu(x), \quad A \in \mathcal{B}
$$

$$
\nu(A)=\int_{V}\left(\int_{A} c_{x y} d \mu(y)\right) d \mu(x) .
$$

Lemma 2.20. The measure $\nu(V)$ is finite if and only if $c \in L^{1}(\mu)$. The measures $\mu$ and $\nu$ are equivalent and

$$
\frac{d \nu}{d \mu}(x)=c(x), \quad x \in V .
$$

Proof. These assertions follow from (2.27):

$$
\begin{aligned}
\nu(A) & =\int_{V}\left(\int_{A} c_{x y} d \mu(y)\right) d \mu(x) \\
& =\int_{A} \int_{V} c_{x y} d \mu(x) d \mu(y) \\
& =\int_{A} c(y) d \mu(y) .
\end{aligned}
$$

Remark 2.21. (1) The operator $R$ is not normalized: $R(\mathbb{1})(x)=c(x)$ where $\mathbb{1}$ is the constant function equal to 1 .

(2) The operator $R$ acts on the space of measures $M(V)$ by the following rule:

$$
(\mu R)(f)=\int_{V} R(f) d \mu
$$

(3) It follows from (2.26) and (2.17) that

$$
\mu R=\nu
$$


Summary. We summarize here the discussion in this subsection. We defined the following objects: a standard measure space $(V, \mathcal{B}, \mu)$, a symmetric set $E$, and a symmetric positive function $c_{x y}: E \rightarrow \mathbb{R}_{+}$. Under some natural assumptions about $E$ and $c_{x y}$, we defined new objects: a symmetric measure $\rho$ with the system of conditional measures $\left(\rho_{x}: x \in V\right)$, a locally integrable function $c(x)$, and a positive operator $R$ such that $d(\mu R)(x)=c(x) d \mu(x)$. In short notation, we have

$$
\left(\mu, c_{x y}\right) \Longrightarrow\left(\rho, \rho_{x}, R\right)
$$

The approach used in Subsection 2.2 gives also the reverse implication: given a symmetric measure $\rho$ on $(V \times V, \mathcal{B} \times \mathcal{B})$ one defines a positive operator $R$ and the measures $\rho_{x}$.

$2^{\text {nd }}$ case: $E$ is a countable Borel equivalence relation. We consider here a particular case when a symmetric Borel subset $E$ is a countable Borel equivalence relation. This means that $E$ is a Borel symmetric subset of $V \times V$ which satisfies the following properties:

(i) $(x, y),(y, z) \in E \Longrightarrow(x, z) \in E$;

(ii) $E_{x}=\{y \in V:(x, y) \in E\}$ is countable for every $x$.

The concept of a countable Borel equivalence relation has been studied extensively last decades in the context of the descriptive set theory, see e.g. JJKL02, Kan08, Kec10

Let $|\cdot|$ be the counting measure on every $E_{x}$. Suppose that $c_{x y}$ is a symmetric function on $E$ such that, for every $x \in V$,

$$
c(x)=\sum_{y \in E_{x}} c_{x y} \in(0, \infty) .
$$

Then we can define the atomic measure $\rho_{x}$ on $V$ by setting

$$
\rho_{x}(A)=\sum_{y \in E_{x} \cap A} c_{x y} .
$$

Finally, define the measure $\rho$ on $E$ :

$$
\rho=\int_{V} \delta_{x} \times \rho_{x} d \mu(x) .
$$

Lemma 2.22. The measure $\rho$ is a symmetric irreducible measure on $E$ which is singular with respect to $\mu \times \mu$.

Proof. Since $(\mu \times \mu)(E)=0$, the singularity of $\rho$ is obvious. It follows from the symmetry of the function $c_{x y}$ and (2.28) that, for $A, B \in \mathcal{B}$,

$$
\begin{aligned}
\rho(A \times B) & =\int_{A} \sum_{y \in E_{x} \cap B} c_{x y} d \mu(x) \\
& =\int_{B} \sum_{x \in E_{y} \cap A} c_{x y} d \mu(y) \\
& =\rho(B \times A) .
\end{aligned}
$$


Definition 2.23. Let $E$ be a countable Borel equivalence relation on a standard Borel space $(V, \mathcal{B})$. A symmetric subset $G \subset$ E is called a graph if $(x, x) \notin G, \forall x \in V$. A graphing of $E$ is a graph $G$ such that the connected components of $G$ are exactly the $E$-equivalence classes. In other words, a graph $G$ generates $E$.

The notion of a graphing is useful for the construction of the path space $\Omega$ related to a Markov process, see Section 4.

The following lemma can be easily proved.

Lemma 2.24. Let $\rho$ be a countable equivalence relation on $(V, \mathcal{B})$, and let $\rho$ be a symmetric measure on $E$. Suppose $G$ is a graphing of $E$. Then $\rho(G)>0$.

For more details regarding integral operators, and analysis of machine learning kernels, the reader may consult the following items [Atk75, CZ07, CWK17, Ho17, JT15a and the papers cited there.

We refer to the following papers regarding the the theory of positive definite kernels [Aro50, AFMP94, PR16], and transfer operators [BJ17, Jor01, JT17a]. The reader will find more references in the papers cited there. Various applications of positive definite kernels are discussed in AJL11, AJV14, AJ14, AJK15, AJ15, AJLM15, AJL17.

\section{LinEAR OPERATORS IN FUNCTIONAL SPACES RELATED TO SYMMETRIC MEASURES}

In this section, we consider several linear operators acting in various functional spaces. Our main focus will be on the basic properties of the graph Laplace and Markov operators. These definitions and results are then used in the subsequent sections.

3.1. Definitions of operators $R, \widetilde{R}, \Delta$, and $P$. The following objects are fixed in this section: $(V, \mathcal{B}, \mu)$ is a $\sigma$-finite measure space; $\rho$ is a symmetric measure on $(V \times V, \mathcal{B} \times \mathcal{B})$, supported by a symmetric subset $E \subset V \times V ; x \mapsto \rho_{x}$ is a measurable family of measures on $(V, \mathcal{B})$ that disintegrates $\rho$. These objects are used in the text below constantly.

Assumption $\boldsymbol{D}$. We will assume that the symmetric measure $\rho$ satisfies the properties:

(a) $c(x)=\rho_{x}(V) \in(0, \infty)$ for $\mu$-a.e. $x \in V$;

(b) the function $c(x)$ belongs to $L_{\text {loc }}^{1}(\mu)$, i.e., $\int_{A} c(x) d \mu(x)<\infty, \forall A \in \mathcal{B}_{\text {fin }}$.

Remark 3.1. (1) One can easily see that the function $c(x)=\rho_{x}(V)$ belongs to $L^{1}(V, \mu)$ if and only if $\rho(E)<\infty$.

(2) We recall that the measure $\nu$ on $V$ is defined by $d \nu(x)=c(x) d \mu(x)$. It is obvious that $L^{2}(\nu)=L^{2}(\mu)$ if and only if there exist $m, M \in(0, \infty)$ such that $m<c(x)<M$ a.e. More general, one can observe that

$$
\nu(A)<\infty \Longleftrightarrow \int_{A} c d \mu<\infty .
$$


Therefore, if $c \in L_{\mathrm{loc}}^{1}(\mu)$, then $\mathcal{B}_{\text {fin }}(\mu) \subset \mathcal{B}_{\text {fin }}(\nu)$.

(3) In general, $\nu$ is an infinite $\sigma$-finite measure, and $\nu$ is finite if and only if $c \in L^{1}(\mu)$.

We now introduce several linear operators. They are defined on the space of Borel functions $\mathcal{F}(V, \mathcal{B})$. In fact, a rigorous definition would require an exact description of the domains and ranges of these operators. We intend to do this when we study realizations of these operators in some Hilbert spaces.

Definition 3.2. Let $(V, \mathcal{B}, \mu), \rho$, and $x \mapsto \rho_{x}$ be as above. The graph Laplace operator is defined on the space of Borel functions $\mathcal{F}(V, \mathcal{B})$ by the formula

$$
\Delta(f)(x)=\int_{V}(f(x)-f(y)) d \rho_{x}(y) .
$$

A function $h \in \mathcal{F}(V, \mathcal{B})$ is called harmonic for the graph Laplace operator $\Delta$ if $\Delta h=0$. The set of harmonic functions is denoted Harm.

When the operator $\Delta$ is considered as an operator acting in the space $L^{2}$, or in the energy space $\mathcal{H}_{E}$, then we use the notation $\mathcal{H a r m}_{2}$ and $\mathcal{H a r m}_{\mathcal{H}}$, respectively, for the corresponding sets of harmonic functions.

It is obvious that every constant function is harmonic. The problem about the existence of nontrivial harmonic functions is extremely important. We will discuss this problem in Sections 4 and 6 .

The most important realizations of $\Delta$ are the corresponding linear operators acting in the Hilbert space $L^{2}(\mu)$ and the energy space $\mathcal{H}_{E}$ (see the definition of $\mathcal{H}_{E}$ below). These realizations are discussed in Sections 7 and 8 .

In Section 2, we already used the positive operator $R$ acting on the space of Borel functions $\mathcal{F}(V, \mathcal{B})$ by the formula:

$$
R(f)(x)=\int_{V} f(y) d \rho_{x}(y)
$$

In the following sections we will work with a Markov operator $P$ and a graph Laplacian $\Delta$ which can be defined by means of the operator $R$. The definition of $\Delta$ is written in the following form:

$$
\Delta(f)(x)=f(x) \int_{V} d \rho_{x}(y)-\int_{V} f(y) d \rho_{x}(y)
$$

and

$$
\Delta(f)(x)=c(x) f(f)-R(f)(x)=(c I-R)(f)(x) .
$$

Define now the operator $P$ by setting

$$
P(f)(x):=\frac{1}{c(x)} R(f)(x) .
$$

This definition can be given in more precise terms as follows:

$$
P(f)(x)=\int_{V} f(y) d \bar{\rho}_{x}(y)
$$


where $d \bar{\rho}_{x}=c(x)^{-1} d \rho_{x}(y)$ is the probability measure obtained from $\rho_{x}$ by normalization. In other words, we define the measure $\bar{\rho}$ on $(V \times V, \mathcal{B} \times \mathcal{B})$ by letting $d \bar{\rho}(x, y)=c(x) d \rho(x, y)$. Then (3.2) is written as

$$
\Delta(f)=c(I-P)(f) .
$$

This formula will be constantly used in the next sections. We observe that relation (3.4) gives an equivalent definition of harmonic functions: a Borel function $h$ is harmonic if $P h=h$.

Together with $R$, we consider another linear operator $\widetilde{R}$ defined on $\mathcal{F}(V \times V, \mathcal{B} \times$ $\mathcal{B})$ :

$$
\widetilde{R}: f \mapsto\left(x \mapsto \rho_{x}(f)\right)
$$

or, equivalently,

$$
\widetilde{R}(f)(x)=\int_{V} f(x, y) d \rho_{x}(y) .
$$

If $d \rho_{x}(y)=c_{x y} d \mu(y)$ (that is $\rho$ is equivalent to $\mu \times \mu$ ), then

$$
\widetilde{R}(f)(x)=\int_{V} c_{x y} f(x, y) d \mu(y) .
$$

Clearly, $\widetilde{R}$ is a positive operator in the following sense: if $f \geq 0$, then $\widetilde{R}(f) \geq 0$.

It is worth noting that similar operators are considered in various areas, e.g. in the theory of graphons Jan13, Lov12.

3.2. A few facts about the operators $R, \widetilde{R}$. In the following theorem, we collected the properties of operators $R, \widetilde{R}$ acting in $L^{p}$-spaces, $p=1,2$. The other two operators, $\Delta$ and $P$, are studied in the next sections.

Theorem 3.3. (1) The operator $\widetilde{R}$ maps $L^{1}(E, \rho)$ onto $L^{1}(V, \mu)$. For any integrable function $f$ on $(E, \rho)$, the relation

$$
\rho(f)=\mu(\widetilde{R}(f))
$$

holds. $\widetilde{R}$ is not one-to-one.

(2) If $g \in L^{\infty}(\mu)$ and $\pi:(x, y) \mapsto x$ is the projection from $V \times V$ to $V$, then

$$
\widetilde{R}((g \circ \pi) f)=g \widetilde{R}(f)
$$

for any $f \in L^{1}(E, \rho)$.

(3) The operator $\widetilde{R}: L^{2}(c \rho) \rightarrow L^{2}(\mu)$ is bounded and

$$
\|\widetilde{R}\|_{L^{2}(c \rho) \rightarrow L^{2}(\mu)} \leq 1 \text {. }
$$

(4) If $c \in L^{\infty}(\mu)$, then $\widetilde{R}$ is a bounded operator from $L^{2}(\rho)$ to $L^{2}(\mu)$ and

$$
\|\widetilde{R}\|_{L^{2}(\rho) \rightarrow L^{2}(\mu)} \leq\|c\|_{\infty} .
$$

(5) For $\pi: E \rightarrow V: \pi(x, y)=x$, let $U_{\pi}: L^{p}(\nu) \rightarrow L^{p}(\rho)$ be the operator acting by the formula $U_{\pi}: f(x) \mapsto \widetilde{f}(x, y)$ where $\widetilde{f}(x, y)=(f \circ \pi)(x, y)$. Then $U_{\pi}$ is an isometric operator for $p=1,2$. 
(6) Suppose the function $c(x)$ is such that $L^{2}(\mu)=L^{2}(\nu)$. Then $L^{2}(c \rho)=L^{2}(\rho)$ and, for any functions $f \in L^{2}(\mu)$ and $g \in L^{2}(\rho)$, the relation

$$
\left\langle U_{\pi} f, g\right\rangle_{L^{2}(\rho)}=\langle f, \widetilde{R}(g)\rangle_{L^{2}(\mu)}
$$

holds. In other words, $\widetilde{R}=U_{\pi}^{*}$ and the operator $\widetilde{R}$ is a co-isometry.

(7) Let $\Pi_{x}: f(x, y) \mapsto f_{x}(y)$ be the restriction of $f(x, y)$ onto $\{x\} \times V$. Then, for any $f \in L^{p}(\rho), p=1,2$,

$$
\widetilde{R}(f)(x)=\left(R \circ \Pi_{x}\right)(f)(x) .
$$

(8) Suppose that $c \in L^{\infty}(\mu)$. Then $R: L^{2}(\mu) \rightarrow L^{2}(\mu)$ is a bounded operator, and

$$
\|R\|_{L^{2}(\mu) \rightarrow L^{2}(\mu)} \leq\|c\|_{\infty} .
$$

(9) Suppose that the function $x \mapsto \rho_{x}(A) \in L^{2}(\mu)$ for any $A \in \mathcal{B}_{\text {fin }}$. Then $R$ is a symmetric unbounded operator in $L^{2}(\mu)$, i.e.,

$$
\langle g, R(f)\rangle_{L^{2}(\mu)}=\langle R(g), f\rangle_{L^{2}(\mu)} .
$$

(10) The operator $R: L^{1}(\nu) \rightarrow L^{1}(\mu)$ is contractive, i.e.,

$$
\|R(f)\|_{L^{1}(\mu)} \leq\|f\|_{L^{1}(\nu)}, \quad f \in L^{1}(\nu) .
$$

Moreover, for any function $f \in L^{1}(\nu)$, the formula

$$
\int_{V} R(f) d \mu(x)=\int_{V} f(x) c(x) d \mu(x)
$$

holds. In other words, $\nu=\mu R$ and

$$
\frac{d(\mu R)}{d \mu}(x)=c(x)
$$

Proof. We will not prove every statement of this theorem with complete details. A part of these results can be easily deduced from the definitions given above.

For (1), we compute using (2.22):

$$
\begin{aligned}
\rho(f) & =\int_{E} f(x, y) d \rho(x, y) \\
& =\int_{V}\left(\int_{V} f(x, y) d \rho_{x}(y)\right) d \mu(x) \\
& =\int_{V} \widetilde{R}(f)(x) d \mu(x) \\
& =\mu(\widetilde{R}(f)) .
\end{aligned}
$$

It follows from the proved relation that the condition $f \in L^{1}(E, \rho)$ implies $\widetilde{R}(|f|) \in$ $L^{1}(V, \mu)$, i.e, $\int_{V} \widetilde{R}(|f|) d \mu<\infty$. Therefore, $\widetilde{R}(f) \in L^{1}(V, \mu)$ because

$$
\int_{V}|\widetilde{R}(f)| d \mu \leq \int_{V} \widetilde{R}(|f|) d \mu
$$


To see that $\widetilde{R}$ is onto $L^{1}(\mu)$, it suffices to consider this operator on characteristic functions over $(V \times V, \mathcal{B} \times \mathcal{B})$. The image of the set of these functions is dense in $L^{1}(\mu)$.

On the other hand, $\widetilde{R}$ is not one-to-one because the kernel of $\widetilde{R}$ is not trivial. In particular,one can find distinct functions $f_{1}(x, y)=\varphi_{1}(x) \psi_{1}(y)$ and $f_{2}(x, y)=$ $\varphi_{2}(x) \psi_{2}(y)$ such that $\widetilde{R}\left(f_{1}\right)(x)=\widetilde{R}\left(f_{2}\right)(x)$.

(2) The result follows from the relation

$$
\begin{aligned}
\widetilde{R}((g \circ \pi) f)(x) & =\int_{V} g(\pi(x, y)) f(x, y) d \rho_{x}(y) \\
& =g(x) \int_{V} f(x, y) d \rho_{x}(y) \\
& =g(x) \widetilde{R}(f)(x) .
\end{aligned}
$$

(3) Suppose $f \in L^{2}(c \rho)$. Because of Theorem 2.8, we obtain that $L^{2}(c \rho)$ is represented as the direct integral of Hilbert spaces $L^{2}\left(c \rho_{x}\right)$ over the measure space $(V, \mathcal{B}, \mu)$. Moreover, $f \in L^{2}(c \rho)$ if and only if the following conditions hold:

(i) $f(x, \cdot) \in L^{2}\left(c \rho_{x}\right)$ for $\mu$-a.e. $x \in V$,

(ii) the function $x \mapsto\|f(x, \cdot)\|_{c(x) \rho_{x}}^{2}$ is in $L^{1}(\mu)$.

Since $c(x)$ is positive and finite a.e., we conclude that $f(x, \cdot) \in L^{2}\left(c \rho_{x}\right)$ if and only if $f(x, \cdot) \in L^{2}\left(\rho_{x}\right)$.

We claim that $\widetilde{R}(f) \in L^{2}(\mu)$. Indeed, using the Schwarz inequality, we obtain

$$
\begin{aligned}
\|\widetilde{R}(f)\|_{L^{2}(\mu)}^{2} & =\int_{V} \widetilde{R}(f)^{2} d \mu \\
& =\int_{V}\left(\int_{V} f(x, y) d \rho_{x}(y)\right)^{2} d \mu(x) \\
& \leq \int_{V}\left(\int_{V} f(x, y)^{2} d \rho_{x}(y)\right)\left(\int_{V} d \rho_{x}(y)\right) d \mu(x) \\
& =\int_{V}\left(\int_{V} f^{2}(x, y) c(x) d \rho_{x}(y)\right) d \mu(x) \\
& =\int_{E} f^{2}(x, y) c(x) d \rho(x, y) \\
& =\|f\|_{L^{2}(c \rho)}^{2} .
\end{aligned}
$$

It shows that $\|\widetilde{R}\|_{L^{2}(c \rho) \rightarrow L^{2}(\mu)} \leq 1$, and the assertion is proved.

(4) This statement can be proved similarly to (3). 
(5) The result follows from the equality

$$
\begin{aligned}
\left\|U_{\pi}(f)\right\|_{L^{2}(\rho)}^{2} & =\int_{E}(f \circ \pi)^{2}(x, y) d \rho(x, y) \\
& =\int_{V} f^{2}(x) c(x) d \mu(x) \\
& =\|f\|_{L^{2}(\nu)}^{2}
\end{aligned}
$$

because $\rho_{x}(V)=c(x)$. A similar equality gives the condition

$$
\left\|U_{\pi}(f)\right\|_{L^{1}(\rho)}^{2}=\|f\|_{L^{1}(\nu)}^{2}
$$

(6) It is easy to see that the assumption $L^{2}(\mu)=L^{2}(\nu)$ is equivalent to the equality $L^{2}(c \rho)=L^{2}(\rho)$. Then $\widetilde{R}$ can be viewed as an operator from $L^{2}(\rho)$ onto $L^{2}(\mu)$, and we calculate, for $f \in L^{2}(\mu), g \in L^{2}(\rho)$,

$$
\begin{aligned}
\langle f, \widetilde{R}(g)\rangle_{L^{2}(\mu)} & =\int_{V} f(x) \widetilde{R}(g)(x) d \mu(x) \\
& =\int_{V} f(x)\left(\int_{V} g(x, y) d \rho_{x}(y)\right) d \mu(x) \\
& =\int_{E}(f \circ \pi)(x, y) g(x, y) d \rho(x, y) \\
& =\left\langle U_{\pi} f, g\right\rangle_{L^{2}(\rho)} .
\end{aligned}
$$

This proves that $\widetilde{R}=U_{\pi}^{*}$, so that $\widetilde{R}$ is a co-isometry.

We note that it follows from this relation that the operator $\widetilde{R}$ is onto $L^{2}(\mu)$. Indeed, we use that $\operatorname{Range}(\widetilde{R})^{\perp}=\operatorname{Ker}\left(\widetilde{R}^{*}\right)$. Since $\widetilde{R}^{*}$ is one-to-one, it has the trivial kernel, and $\widetilde{R}$ is onto.

(7) This is obvious. 
(8) In order to prove this assertion, we apply Jensen's inequality for the probability measure $d \bar{\rho}_{x}=c(x)^{-1} d \rho_{x}$. Then, for any $f \in L^{2}(\mu)$ we have,

$$
\begin{aligned}
\int_{V}[R(f)(x)]^{2} d \mu(x) & =\int_{V}\left(\int_{V} f(y) c(x) d \bar{\rho}_{x}(y)\right)^{2} d \mu(x) \\
& \leq \int_{V}\left(\int_{V} f^{2}(y) c^{2}(x) d \bar{\rho}_{x}(y)\right) d \mu(x) \\
& =\int_{V}\left(\int_{V} f^{2}(y) c(x) d \rho_{x}(y)\right) d \mu(x) \\
& \leq\|c\|_{\infty} \iint_{V \times V} f^{2}(y) d \rho(x, y) \quad(\rho \text { is symmetric }) \\
& =\|c\|_{\infty} \iint_{V \times V} f^{2}(x) d \rho(x, y) \quad\left(\rho_{x}(V)=c(x)\right) \\
& =\|c\|_{\infty} \iint_{V} c(x) f^{2}(x) d \mu(x) \\
& \leq\|c\|_{\infty}^{2} \int_{V} f^{2}(x) d \mu(x)
\end{aligned}
$$

Hence, $R(f) \in L^{2}(\mu)$, and the norm of $R$ in $L^{2}(\mu)$ is bounded by $\|c\|_{\infty}$. We note that if $c \notin L^{\infty}(\mu)$, then $R$ is, in general, an unbounded operator.

(9) We first observe that the assumption that $\rho_{x}(A) \in L^{2}(\mu), A \in \mathcal{B}_{\text {fin }}$, means that $R$ is a densely defined operator. Then, to show that $R$ is symmetric, we use the fact that the measure $\rho$ is symmetric:

$$
\begin{aligned}
\langle g, R(f)\rangle_{L^{2}(\mu)} & =\int_{V} g(x)\left(\int_{V} f(y) d \rho_{x}(y)\right) d \mu(x) \\
& =\iint_{V \times V} f(y) g(x) d \rho(x, y) \\
& =\int_{V} f(y)\left(\int_{V} g(x) d \rho_{y}(x)\right) d \mu(y) \\
& =\int_{V} f(y) R(g)(y) d \mu(y) \\
& =\langle R(g), f\rangle_{L^{2}(\mu)} .
\end{aligned}
$$

(10) We compute

$$
\begin{aligned}
\|R(f)\|_{L^{1}(\mu)} & =\int_{V}\left|\int_{V} f(y) d \rho_{x}(y)\right| d \mu(x) \\
& \leq \int_{V} \int_{V}|f(y)| d \rho_{x}(y) d \mu(x) \\
& =\int_{V}|f(y)| c(y) d \mu(y) \\
& =\|f\|_{L^{1}(\nu)} .
\end{aligned}
$$


The other statement in (10) is obvious.

\section{Markov processes associated With SymmetriC MeAsures}

In this section, we introduce a Markov process related to a symmetric measure $\rho$ on $(V \times V, \mathcal{B} \times \mathcal{B})$ and generated by a Markov operator.

4.1. Markov operators. By a Markov operator, we mean a positive self-adjoint operator $P$ in a $L^{2}$-space satisfying the normalization condition $P(\mathbb{1})=\mathbb{1}$. The book [Rev84] is a remarkable introduction to homogeneous Markov chains with measurable state space. More information about various aspects of Markov chains can be found in the following papers: [BJ15b, DJ06, DJ06, GQ15, JP12, KM15, Luk16.

An example of a Markov operator built by a symmetric measure has been given in Section 3. We consider here the main properties of the operator $P$ defined above in (3.3).

Definition 4.1. Let $(V, \mathcal{B}, \mu)$ be a a measure space, $E$ a symmetric subset of $V \times V$, and $\rho$ a symmetric measure with support $E$. Let $R$ be a positive operator defined by $\rho$ as in (2.17). We set

$$
P(f)(x)=\frac{1}{c(x)} R(f)(x)
$$

or

$$
P(f)(x)=\frac{1}{c(x)} \int_{V} f(y) d \rho_{x}(y)=\int_{V} f(y) P(x, d y)
$$

where $P(x, d y)$ is the probability measure obtained by normalization of $\rho_{x}$.

Remark 4.2. (1) Because every measure $P(x, \cdot)$ is probability, the positive operator $P$ is obviously normalized, i.e., $P(\mathbb{1})=\mathbb{1}$. In fact, $P$ is defined on a set of Borel functions over $(V, \mathcal{B})$ (allowing functions with infinite values). Our main interest in the operator $P$ is focused on the properties of $P$ as an operator on the spaces $L^{2}(\mu)$ and $L^{2}(\nu)$.

(2) It is worth noting that if a measurable field of probability measures $x \rightarrow$ $\mu(x), x \in V$, is given on the space $(V, \mathcal{B})$, then there exists a normalized positive operator $P$ (Markov operator) defined by $x \rightarrow \mu(x)$ similar to (4.1). But the converse is not true: not every Markov operator determines a measurable field of probability measures.

(3) Working with a positive normalized operator $P$, we will often use Jensen's inequality which states that $(P(f))^{2} \leq P\left(f^{2}\right)$ for any Borel function $f$.

(4) The notation $P(x, d y)$ for the measure $\bar{\rho}_{x}=c(x)^{-1} \rho_{x}$ is used in (4.1) for consistency with notations common in the literature on Markov processes.

(5) If $d \rho_{x}(y)=c_{x y} d \mu(y)$, then the operator $P$ is defined by its density

$$
P(f)(x)=\int_{V} p(x, y) f(y) d \mu(y)
$$


where

$$
p(x, y)=\frac{c_{x y}}{c(x)} .
$$

It follows from the definition that $p(x, y)>0$ for any $(x, y) \in E$, and

$$
\int_{V} p(x, y) d \mu(y)=1, \quad \forall x \in V .
$$

This simple fact makes clear parallels with Markov processes defined on discrete electrical networks.

In the following result, we show how the operator $P$ acts on the measures $\nu$ and $\mu$.

Lemma 4.3. (1) Let $d \nu(x)=c(x) d \mu(x)$ where $c(x)=\rho_{x}(V)$. Then $\nu P=\nu$.

$$
\frac{d \mu P}{d \mu}(x)=\int_{V} \frac{1}{c(y)} d \rho_{x}(y) .
$$

Proof. (1) We use (4.1) and the symmetry of $\rho$ (see (2.16) ) to compute the RadonNikodym derivative $\frac{d(\nu P)}{d \nu}$. Let $f$ be any Borel function over $(V, \mathcal{B})$, then

$$
\begin{aligned}
\int_{V} P(f) d \nu & =\int_{V}\left(\frac{1}{c(x)} \int_{V} f(y) d \rho_{x}(y)\right) c(x) d \mu(x) \\
& \left.=\int_{V} f(y)\left(\int_{V} d \rho_{y}(x)\right) d \mu(y) \quad \text { (by symmetry of } \rho\right) \\
& =\int_{V} f(y) c(y) d \mu(y) \\
& =\int_{V} f d \nu .
\end{aligned}
$$

(2) To prove the second assertion, we need to find a measurable function $g(x)$ such that

For this,

$$
\int_{V} P(f) d \mu=\int_{V} f g d \mu
$$

$$
\begin{aligned}
\int_{V} P(f) d \mu & =\int_{V}\left(\int_{V} f(y) P(x, d y)\right) d \mu(x) \\
& =\iint_{V \times V} \frac{f(y)}{c(x)} d \rho_{x}(y) d \mu(x) \\
& =\int_{V} f(x)\left(\int_{V} \frac{1}{c(y)} d \rho_{x}(y)\right) d \mu(x) .
\end{aligned}
$$

This proves (2).

The following theorem contains several basic properties of the operator $P$. The most important is its self-adjointess in $L^{2}(\nu)$. 
Theorem 4.4. Let $d \nu(x)=c(x) d \mu(x)$ be the $\sigma$-finite measure on $(V, \mathcal{B})$ where $\mu$ and $c$ are defined as above. Suppose $P$ is defined by (4.1). Then:

(1) The bounded operator $P: L^{2}(\nu) \rightarrow L^{2}(\nu)$ is self-adjoint.

(2) The operator $P$ considered in the spaces $L^{2}(\nu)$ and $L^{1}(\nu)$ is contractive, i.e.,

$$
\|P(f)\|_{L^{2}(\nu)} \leq\|f\|_{L^{2}(\nu)}, \quad\|P(f)\|_{L^{1}(\nu)} \leq\|f\|_{L^{1}(\nu)} .
$$

(3) Spectrum of $P$ is a subset of $[-1,1]$.

(4) Suppose that $(V, \mathcal{B}, \mu)$ is a probability measure space and the operator $P$ is defined by (4.2). Then $P$ is contractive in $L^{2}(\mu)$.

We remark that, in fact, $P$ is also a contraction in the space $L^{p}(\nu), 1 \leq p \leq \infty$, but we will not use this in the paper.

Proof. To see that (1) holds, we use Theorem 3.3 (6) and formula (4.1): for any $f, g \in L^{2}(\nu)$

$$
\begin{aligned}
\langle P(f), g\rangle_{L^{2}(\nu)} & =\left\langle c^{-1} R(f), g\right\rangle_{L^{2}(\nu)} \\
& =\langle R(f), g\rangle_{L^{2}(\mu)} \\
& =\langle f, R(g)\rangle_{L^{2}(\mu)} \\
& =\langle f, c P(g)\rangle_{L^{2}(\mu)} \\
& =\langle f, P(g)\rangle_{L^{2}(\nu)}
\end{aligned}
$$

The proof of (2) follows from the inequalities $P(f)^{2} \leq P\left(f^{2}\right)$ and $|P(f)| \leq P(|f|)$ and the following calculation:

$$
\begin{aligned}
\int_{V} P(f)^{2}(x) c(x) d \mu(x) & \leq \int_{V} P\left(f^{2}\right)(x) c(x) d \mu(x) \\
& =\int_{V} R\left(f^{2}\right)(x) d \mu(x) \\
& =\int_{V} \int_{V} f^{2}(y) d \rho_{x}(y) d \mu(x) \\
& \left.=\iint_{V \times V} f^{2}(y) d \rho(x, y) \quad \text { (by symmetry of } \rho\right) \\
& =\iint_{V \times V} f^{2}(x) d \rho(x, y) \quad \\
& =\int_{V} f^{2}(x)\left(\int_{V} d \rho_{x}(y)\right) d \mu(x) \\
& =\int_{V} f^{2}(x) c(x) d \mu(x) .
\end{aligned}
$$

Similarly,

$$
\int_{V}|P(f)(x)| d \nu(x) \leq \int_{V} P(|f|)(x) d \nu(x)=\int_{V}|f(x)| d \nu(x)
$$

since $\nu$ is $P$-invariant.

Assertion (3) is now a direct consequence of the proved statements (1) and (2). 
To see that (4) holds, we use the Schwarz inequality and the fact that $0 \leq$ $p(x, y) \leq 1$ :

$$
\begin{aligned}
\int_{V} P(f)^{2}(x) d \mu(x) & =\int_{V}\left(\int_{V} p(x, y) f(y) d \mu(y)\right)^{2} d \mu(x) \\
& \leq \int_{V}\left(\int_{V} p(x, y)^{2} d \mu(y)\right)\left(\int_{V} f(y)^{2} d \mu(y)\right) d \mu(x) \\
& \leq\|f\|_{L^{2}(\mu)}^{2} \int_{V}\left(\int_{V} p(x, y) d \mu(y)\right) d \mu(x) \\
& =\|f\|_{L^{2}(\mu)}^{2} .
\end{aligned}
$$

Hence,

$$
\|P(f)\|_{L^{2}(\mu)} \leq\|f\|_{L^{2}(\mu)} .
$$

It is useful to represent a symmetric measure $\rho$ via a Markov operator $P$.

Lemma 4.5. Let $(V, \mathcal{B}, \mu)$ be a measure space and let $\nu=c \mu$. A Borel $\sigma$-finite measure $\rho$ on $(V \times V, \mathcal{B} \times \mathcal{B})$ is symmetric if and only if there exists a self-adjoint Markov operator $P$ on $L^{2}(\nu)$ such that, for any $A, B \in \mathcal{B}_{\text {fin }}$,

$$
\rho(A \times B)=\int_{V} \chi_{A} P\left(\chi_{B}\right) d \nu=\left\langle\chi_{A}, P\left(\chi_{B}\right)\right\rangle_{L^{2}(\nu)} .
$$

More generally, such an operator $P$ defines a sequence of symmetric measures $\left(\rho_{n}\right)_{n \in \mathbb{N}}$ by the formula

$$
\rho_{n}(A \times B)=\left\langle\chi_{A}, P^{n}\left(\chi_{B}\right)\right\rangle_{L^{2}(\nu)} .
$$

Proof. This results follows from Proposition 2.13 and the definition of $P$ and $\nu$.

It follows from Theorem 4.4 that $P^{n}$ is self-adjoint for every $n$, and therefore $\rho_{n}$ is a well defined symmetric measure. One can see that, for any $n \in \mathbb{N}$,

$$
d \rho_{n}(x, y)=c(x) P_{n}(x, d y) d \mu(x)
$$

and

$\iint_{V \times V} f(x, y) d \rho_{n}(x, y)=\iint_{V \times V} f(x, y) c(x) P_{n}(x, d y) d \mu(x)=\int_{V} P^{n}(f)(x) d \nu(x)$.

4.2. Harmonic functions for $P$. In this part we will deal with Markov operators $P$ in $L^{2}(\nu)$ preserving the measure $\nu$.

Let $P$ be the Markov operator defined by (4.1). In other words, this operator is define by an irreducible symmetric measure $\rho$. We recall that, as $P$ is a positive operator such that $P(\mathbb{1})=1$, then for any function $f$ the inequality $P(f)^{2}(x) \leq$ $P\left(f^{2}\right)(x)$ holds a.e. We need a stronger form of this inequality. 
Lemma 4.6. For the Markov operator

$$
P(f)(x)=\int_{V} f(y) P(x, d y)
$$

and any non-constant function $f \in L^{2}(\nu)$, there exists a subset $A \subset V$ of positive measure $\nu$ such that $P(f)^{2}(x)<P\left(f^{2}\right)(x), x \in A$.

Proof. The proof follows from Jensen's inequality applied to the convex function $\varphi(x)=x^{2}$. As shown in the proof of [Rud87, Theorem 3.3], the equality occurs only for affine convex functions.

Theorem 4.7. Let $(V, \mathcal{B}, \nu)$ be a measure space with finite or $\sigma$-finite measure. Suppose $P$ is a Markov operator on $L^{2}(\nu)$ defined by an irreducible symmetric measure according to (4.1). Then

$$
L^{2}(\nu) \cap \mathcal{H a r m}(P)= \begin{cases}0, & \nu(V)=\infty \\ \mathbb{R} \mathbb{1}, & \nu(V)<\infty\end{cases}
$$

where $\mathbb{R} \mathbb{1}$ is the set of constant functions. Moreover, 1 does not belong to the point spectrum of the operator $P$ on the space $L^{2}(\nu)$.

Proof. If we show that there is no nontrivial harmonic functions in $L^{2}(\nu)$, then we prove that 1 is not an eigenvalue for $P$. Clearly, the converse also holds.

Assume for contrary that there exists a non-constant function $f \in L^{2}(\nu)$ such that $P(f)=f$. Then, by Lemmas 4.3 and 4.6, we have

$$
\begin{aligned}
\|f\|_{L^{2}(\nu)}^{2} & =\int_{V} f(x)^{2} d \nu(x) \\
& =\int_{V}(P(f))^{2}(x) d \nu(x) \\
& <\int_{V} P\left(f^{2}\right)(x) d \nu(x) \\
& =\int_{V} f^{2}(x) d(\nu P)(x) \\
& =\int_{V} f^{2}(x) d \nu(x) \\
& =\|f\|_{L^{2}(\nu)}^{2} .
\end{aligned}
$$

This contradiction proves the theorem.

We recall that if $T$ is a contraction in a Hilbert space $\mathcal{K}$, then $\mathcal{K}$ is decomposed into the orthogonal direct sum

$$
\mathcal{K}=\operatorname{Fix}(T) \oplus \overline{\operatorname{Range}(I-T)} .
$$

This is a form of the mean ergodic theorem, see e.g., [EFHN15, Theorem 8.6].

We apply this result to the case of an abstract Markov operator $P$ acting in $L^{2}(\nu)$ such that $\nu P=\nu$. We assume here that $P$ is self-adjoint and contractive. Denote by 
$C b(P)$ the subset of $L^{2}(\nu)$ formed by $P$-coboundaries, i.e., $C b(P)=\{g-P(g) \mid g \in$ $\left.L^{2}(\nu)\right\}$. Clearly, for any function $g \in L^{2}(\nu)$, one has $\int_{V}(g-P(g)) d \nu=0$ since $\nu$ is $P$-invariant. Hence

$$
C b(P) \subset L_{0}^{1}(\nu) \cap L^{2}(\nu)=\left\{f \in L^{2}(\nu): \int_{V} f d \nu=0\right\} .
$$

Proposition 4.8. (1) Let $P$ be a self-adjoint contractive operator on $L^{2}(\nu)$ satisfying $\nu P=\nu$. Then

$$
L^{2}(\nu)=\operatorname{Harm}_{2}(P) \oplus \overline{C b(P)},
$$

where $\mathcal{H a r m}_{2}(P)=\left\{f \in L^{2}(\nu): P(f)=f\right\}$ and $\overline{C b(P)}$ is the closure of $C b(P)$ in $L^{2}(\nu)$.

(2) Suppose that $P$ is a Markov operator defined by an irredusible symmetric measure $\rho$ as in Theorem 4.7. Then the set $\left\{g-P(g) \mid g \in L^{2}(\nu)\right\}$ of P-coboundaries is dense in $L^{2}(\nu)$.

(3) The operator $(I-P)^{-1}$ is unbounded in $L^{2}(\nu)$.

Proof. (1) Clearly, this statement is a form of (4.4). Suppose that $f$ is a function from $L^{2}(\nu) \ominus L_{0}^{2}(\nu)$. Then, for arbitrary $g \in L^{2}(\nu)$,

$$
\begin{aligned}
0 & =\langle f, g-P(g)\rangle_{L^{2}(\nu)} \\
& =\langle f, g\rangle_{L^{2}(\nu)}-\langle f, P(g)\rangle_{L^{2}(\nu)} \\
& =\langle f, g\rangle_{L^{2}(\nu)}-\langle P(f), g\rangle_{L^{2}(\nu)} \\
& =\langle f-P(f), g\rangle_{L^{2}(\nu)} .
\end{aligned}
$$

Hence, $f=P(f)$. The same proof shows that if $f \in \mathcal{H a r m}_{2}(P)$, then $\langle f, g\rangle_{L^{2}(\nu)}=$ $\langle P(f), g\rangle_{L^{2}(\nu)}=\langle f, P(g)\rangle_{L^{2}(\nu)}$ and $f \perp C b(P)$.

(2) The result follows from Theorem 4.7 and statement (1) of this theorem.

(3) This observation follows from (2).

In the next statement we summarize facts about harmonic functions in $L^{2}(\nu)$.

Theorem 4.9. Let $P$ be a self-adjoint contractive operator on $L^{2}(\nu)$ such that $\nu P=\nu$. The following are equivalent:

(i) $\lambda=1$ is not an eigenvalue for the operator $P$ in $L^{2}(\nu)$;

(ii) $\{P(f)=f\} \cap L^{2}(\nu)=0$;

(iii)

$$
\lim _{N \rightarrow \infty} \frac{1}{N} \sum_{n=1}^{N} P^{n}(f)=0, \quad f \in L^{2}(\nu) ;
$$

(iv) for any $A, B \in \mathcal{B}_{\text {fin }}$,

$$
\lim _{N \rightarrow \infty} \frac{1}{N} \sum_{n=1}^{N} \rho_{n}(A \times B)=0 .
$$

Proof. (i) $\Longleftrightarrow$ (ii). This equivalence is a reformulation of the proved results from Theorems 4.4 and 4.7 
(ii) $\Longleftrightarrow$ (iii). By the mean ergodic theorem for contractive operators (see, for example, Yos95, EFHN15]), we obtain that, for any vector $f \in L^{2}(\nu)$, the sequence of vectors

$$
S_{N}(f)=\frac{1}{N} \sum_{n=1}^{N} P^{n}(f)
$$

converges strongly to a vector $\varphi$ that belongs to the closed subspace of $P$-invariant vectors, i.e., $\varphi$ must be a harmonic function. the converse statement is obviously true.

(iii) $\Longleftrightarrow$ (iv). We observe that the strong converges of $S_{N}(f)$ is equivalent to the weak converges. Then, for characteristic functions $\chi_{A}, \chi_{B}, A, B \in \mathcal{B}_{\text {fin }}$, we find that

$$
\begin{aligned}
\left\langle S_{N}\left(\chi_{A}\right), \chi_{B}\right\rangle_{L^{2}(\nu)}= & \frac{1}{N} \sum_{n=1}^{N}\left\langle\chi_{A}, P^{n}\left(\chi_{B}\right)\right\rangle_{L^{2}(\nu)} \\
= & \frac{1}{N} \sum_{n=1}^{N} \rho_{n}(A \times B) \\
& \longrightarrow 0, \quad N \rightarrow \infty .
\end{aligned}
$$

We used here Lemma 4.5.

In the next proposition, we consider several properties of harmonic functions for a Markov operators $P$ acting on the space of measurable function $\mathcal{F}(V, \mathcal{B}, \nu)$ where $\nu P=\nu$.

Proposition 4.10. Suppose that $P: \mathcal{F}(V, \mathcal{B}, \nu) \rightarrow \mathcal{F}(V, \mathcal{B}, \nu)$ is a positive operator $(f \geq 0 \Longrightarrow P(f) \geq 0)$ such that $P(\mathbb{1})=1$ and $\nu P=\nu$.

(1) If a function $h \in L^{2}(\nu)$ and $P(h)(x)=h(x) \nu$-a.e., then $P\left(h^{2}\right)=h^{2}$ a.e.

(2) If $h, k \in L^{2}(\nu)$ and $P(h)=h, P(k)=k$, then $P(h k)=h k \nu$-a.e.

(3) If $h \in L^{2}(\nu)$ and $P(h)=h$, then $P(h g)=h P(g)$ for any function $g \in \mathcal{F}$.

(4) If $h \in L^{2}(\nu)$ and $P(h)=h$, then $P\left(h^{n}\right)=h^{n}, n \in \mathbb{N}$.

Proof. (1) Since $P$ is a positive and normalized operator, the inequality $P(f)^{2}(x) \leq$ $P\left(f^{2}\right)(x)$ holds for every function $f \in \mathcal{F}$ and every $x$. By assumption, $h \in L^{2}(\nu)$, hence $h^{2} \in L^{1}(\nu)$. Next, since $\nu$ is $P$-invariant, we have

$$
\int_{V} P\left(h^{2}\right) d \nu=\int_{V} h^{2} d \nu
$$

This means that

$$
\begin{aligned}
0 & \leq \int_{V}\left(P\left(h^{2}\right)-P(h)^{2}\right) d \nu \\
& =\int_{V} P\left(h^{2}\right) d \nu-\int_{V} h^{2} d \nu \\
& =0
\end{aligned}
$$

Thus, $P\left(h^{2}\right)=h^{2}$ a.e. 
(2) Fix $x \in V$. Because $P$ is positive, we see that

$$
\langle f, g\rangle_{x}:=P(f g)(x)-P(f)(x) P(g)(x)
$$

is a positive definite bi-linear form. Since $h$ and $k$ are $P$-invariant functions, we obtain, by the Schwarz inequality, that

$$
(P(h k)-h k)^{2} \leq\left(P\left(h^{2}\right)-h^{2}\right)\left(P\left(k^{2}\right)-k^{2}\right),
$$

and this inequality holds for every $x$. Now we can apply (1) and conclude that $P(h k)=h k$.

(3) This statement can be proved similarly to (2).

(4) We use (1) and (3) to deduce (4).

4.3. Markov processes. It is well known that every Markov operator defines a Markov process on a measure space. We will describe this process explicitly for the operator $P$ determined by (4.1).

Recall our setting: $(V, \mathcal{B}, \mu)$ is a $\sigma$-finite measure space, and $\rho$ is a symmetric measure defined by a positive operator $R$ (see Proposition 2.13) such that $c(x)=$ $\rho_{x}(V)$. The measure $\rho$ admits a disintegration (see Section 2) such that

$$
d \rho(x, y)=d \rho_{x}(y) d \mu(x)=P(x, d y) d \nu(x)
$$

where $x \mapsto d \rho_{x}$ is a measurable family of positive measures, and $P(x, d y)$ is the probability measure obtained by normalization of $\rho_{x}$.

Fix a point $x \in V$, and define inductively a sequence of probability measures $\left(P_{n}(x, \cdot): n \in \mathbb{N}_{0}\right)$. For any set $A \in \mathcal{B}$, we define

$$
\begin{aligned}
& P_{0}(x, A)=\chi_{A}(x), \\
& P_{1}(x, A)=\int_{V} P_{0}(y, A) P(x, d y) \\
& \ldots \ldots \ldots \ldots \ldots \ldots \ldots \ldots \ldots \ldots \ldots \ldots \ldots \ldots \\
& P_{n+1}(x, A)=\int_{V} P_{n}\left(x_{n}, A\right) P\left(x, d x_{n}\right),
\end{aligned}
$$

To simplify notation, we write $P(x, A)$ for $P_{1}(x, A)$.

For the reader's convenience we formulate two statements in the next lemma.

Lemma 4.11. (1) Let $x \in V$ be a fixed point. For every $n \in \mathbb{N}_{0}$, the map $\mathcal{B} \ni A \mapsto$ $P_{n}(x, A)$ defines a probability measure on $(V, \mathcal{B})$. For any fixed $A \in \mathcal{B}$, the function $x \mapsto P_{n}(x, A)$ is $\mathcal{B}$-measurable $n \in \mathbb{N}_{0}$.

(2) For $A \in \mathcal{B}$,

$$
P_{n}(x, A)=P^{n}\left(\chi_{A}\right)(x), \quad n \in \mathbb{N}_{0} .
$$

Proof. Statement (1) follows from the definition of $P_{n}(x, A)$, and (2) can be proved by induction. 
Remark 4.12. (i) It is useful to interpret $P_{n}(x, A)$ as the probability to get to a set $A \in \mathcal{B}$ for $n$ steps assuming that the process begins at $x$. In particular, $P_{0}(x, A)=\delta_{A}(x)$. We call $\left(P_{n}(x, A)\right), n \geq 0$, a sequence of transition probabilities.

(ii) In case when the symmetric measure $\rho$ is defined by (2.22), we have the following formulas for $P_{n}(x, A)$ :

$$
\begin{aligned}
& P_{1}(x, A)=\int_{V} \frac{\chi_{A}(y)}{c(x)} d \rho_{x}(y) \\
& P_{n}(x, A)=\int_{V} \cdots \int_{V} \frac{\chi_{A}(y)}{c\left(x_{n-1}\right) \cdots c(x)} d \rho_{x_{n-1}}(y) \cdots d \rho_{x}\left(x_{1}\right) \\
& \text {........................ }
\end{aligned}
$$

We finish this subsection by pointing out a curious relation between discrete Markov chains and continuous Poisson type distributions. The reader can find relevant materials in [AMR15, App09, Kal83]. The reader can find the theory of operator semigroups in the remarkable book [Yos95.

Theorem 4.13. (1) Let $P$ be a Markov operator on $L^{2}(V, \mathcal{B}, \nu)$ and let $\left(P_{n}\right)$ be a discrete Markov process generated by $P$. For every $t \in \mathbb{R}_{+}$and $A \in \mathcal{B}$, define

$$
Q_{t}(x, A):=\sum_{n=0}^{\infty} e^{-\lambda t} \frac{(\lambda t)^{n}}{n !} P_{n}(x, A) .
$$

Then, the distribution $Q_{t}$ satisfies the property:

$$
\int_{V} Q_{s}(y, A) Q_{t}(x, d y)=Q_{t+s}(x, A),
$$

Moreover, $\nu Q_{t}=\nu$ if and only if $\nu P=\nu$.

(2) $\left\{Q_{t}: t \geq 0\right\}$ is a strongly continuous semigroup such that $Q_{0}=I$, and the generator $\mathcal{L}$ of $\left\{Q_{t}: t \geq 0\right\}$ is $\lambda(P-I)$.

(3) The following are equivalent:

(i) $f$ is a harmonic function with respect to $P$;

(ii) $\mathcal{L}(f)=0$;

(iii) $Q_{t}(f)=f$ for $t \geq 0$.

(4) The operators

$$
S_{t}(f)(x):=e^{-c(x) t} \sum_{n=0}^{\infty} \frac{(c(x) t)^{n}}{n !} \int_{V} f(y) P_{n}(x, d y)
$$

form a self-adjoint contractive semigroup of operators in $L^{2}(\mu)$. The generator $\mathcal{L}$ of $\left\{S_{t}\right\}_{t \geq 0}$ is $c(P-I)$, i.e.,

$$
S_{t}=e^{-t \Delta}
$$

where $\Delta$ is considered as an unbounded operator in $L^{2}(\mu)$. 
Proof. In the proof of (1), we use the binomial formula and the relation

$$
P_{n+m}(x, A)=\int_{V} P_{m}(y, A) P_{n}(x, d y)
$$

which follows from Lemma 4.11. Thus, we have

$$
\begin{aligned}
& \int_{V} Q_{s}(y, A) Q_{t}(x, d y) \\
& =\int_{V} \sum_{m=0}^{\infty} e^{-\lambda s} \frac{(\lambda s)^{m}}{m !} P_{m}(x, A) \sum_{n=0}^{\infty} e^{-\lambda t} \frac{(\lambda t)^{n}}{n !} P_{n}(x, d y) \\
& =e^{-\lambda(t+s)} \sum_{m, n=0}^{\infty} \frac{(\lambda s)^{m}}{m !} \frac{(\lambda t)^{n}}{n !} P_{m+n}(x, A) \\
& =e^{-\lambda(t+s)} \sum_{k=0}^{\infty} \sum_{n=0}^{k} \frac{(\lambda s)^{k-n} k !}{(k-n) !} \frac{(\lambda t)^{n}}{n !} \frac{1}{k !} P_{k}(x, A) \\
& =e^{-\lambda(t+s)} \sum_{k=0}^{\infty} \frac{(\lambda(t+s))^{k}}{k !} P_{k}(x, A) \\
& =Q_{t+s}(x, A) .
\end{aligned}
$$

It follows from the definition of $Q_{t}$ that $\nu$ must be invariant with respect to $P$ and $Q_{t}$ simultaneously.

(2) We first note that

$$
Q_{0}(f)(x)=P_{0}(f)(x)=\delta_{x}(f)=f(x)
$$

as follows from (4.5). Moreover, $Q_{t}$ is strongly continuous because $P$ is a bounded operator. Then the generator $\mathcal{L}$ of the semigroup $\left\{Q_{t}: t \geq 0\right\}$ can be found by direct computation:

$$
\begin{aligned}
\frac{d Q_{t}(f)}{d t} & =\sum_{n=0}^{\infty} \lambda e^{-\lambda t}\left(-\frac{(\lambda t)^{n}}{n !}+\frac{(\lambda t)^{n-1}}{(n-1) !}\right) P_{n}(f) \\
& =\lambda(P(f)-f) .
\end{aligned}
$$

Hence, the generator of $\left\{Q_{t}: t \geq 0\right\}$ is

$$
\mathcal{L}(f):=\lim _{t \rightarrow 0} \frac{Q_{t}(f)-f}{t}=\lambda(P-I)(f) .
$$

(3) This statement is an immediate consequence of (1) and (2).

(4) To check that $\left\{S_{t}\right\}$ is a semigroup, we use the same calculation as in (1) applied to (4.6). Statement (2), employed to the semigroup $\left\{S_{t}\right\}$, gives the exact formula for the generator of this semigroup.

Remark 4.14. The same approach can be applied to the study of semigroups of operators, defined as in (4.5) and (4.6), acting in the finite energy space $\mathcal{H}_{E}$, see Sections 6 and 8 . 
The following fact follows directly from Theorem 4.13 ,

Corollary 4.15. Suppose that the measure space $(V, \mathcal{B}, \mu)$ and the measurable field of probability measures $x \mapsto P_{n}(x, \cdot)$ be as above. Let $(X, \mathcal{A}, m)$ be another measure space. For any sets $A \in \mathcal{B}, N \in \mathcal{A}$ and $x \in V$, define

$$
Q_{x}(N, A)=\sum_{n=0}^{\infty} e^{-m(N)} \frac{m(N)^{n}}{n !} P_{n}(x, A) .
$$

Then $x \mapsto Q_{x}(\cdot, \cdot)$ is a measurable field of probability measures on $(X \times V, \mathcal{A} \times \mathcal{B})$.

Path space and measure. We denote by $\Omega$ the infinite Cartesian product $V^{\mathbb{N}_{0}}=V \times V \times \cdots$. Let $\left(X_{n}(\omega): n=0,1, \ldots\right)$ be the sequence of random variables $X_{n}: \Omega \rightarrow V$ such that $X_{n}(\omega)=\omega_{n}$. It is convenient to interpret $\Omega$ as the path space of the Markov process $\left(P_{n}\right)$. Every $\omega \in \Omega$ represents an infinite path, and if $X_{0}(\omega)=x$, then we say that the path $\omega$ begins at $x$. A subset $\left\{\omega \in \Omega: X_{0}(\omega) \in\right.$ $\left.A_{0}, \ldots X_{k}(\omega) \in A_{k}\right\}$ is called a cylinder set defined by $A_{0}, A_{1}, \ldots, A_{k}, k \in \mathbb{N}_{0}$. The collection of cylinder sets generates the $\sigma$-algebra $\mathcal{C}$ of Borel subsets of $\Omega$.

It follows from this definition of Borel structure on $\Omega$ that the function $X_{n}$ : $\Omega \rightarrow V$ is Borel. This construction allows us to define an increasing sequence of $\sigma$-subalgebras $\mathcal{F}_{\leq n}$ such that $\mathcal{F}_{\leq n}$ is the smallest subalgebra for which the functions $X_{0}, X_{1}, \ldots, X_{n}$ are Borel. By $\mathcal{F}_{n}$, we denote the $\sigma$-subalgebra $X_{n}^{-1}(\mathcal{B})$. Since $X_{n}^{-1}(\mathcal{B})$ is a $\sigma$-subalgebra of $\mathcal{C}$, there exists a projection $E_{n}: L^{2}(V, \mathcal{C}, \lambda) \rightarrow$ $L^{2}\left(\Omega, X_{n}^{-1}(\mathcal{B}), \lambda\right)$, where the measure $\lambda$ is defined in Section 5 . The projection $E_{n}$ is called the conditional expectation with respect to $X_{n}^{-1}(\mathcal{B})$. It satisfies the property:

$$
E_{n}\left(f \circ X_{n}\right)=f \circ X_{n} .
$$

Next, we define a measure on $\Omega$. Let $x \in V$ be a fixed point. Then, let $\Omega_{x}$ be the set of infinite paths beginning at $x$ :

$$
\Omega_{x}:=\left\{\omega \in \Omega: X_{0}(\omega)=x\right\} .
$$

Clearly, $\Omega=\coprod_{x \in V} \Omega_{x}$.

Lemma 4.16. For the objects introduced above, there exists a probability measure $\mathbb{P}_{x}$ on $\Omega_{x}$ such that its values on cylinder subsets of $\Omega_{x}$ are determined by the formula:

$$
\begin{aligned}
\mathbb{P}_{x}\left(X_{1} \in A_{1}, \ldots, X_{n} \in A_{n}\right) & =\int_{A_{1}} \cdots \int_{A_{n}} P\left(y_{n-1}, d y_{n}\right) \cdots P\left(x, d y_{1}\right) \\
& =\int_{A_{1}} \cdots \int_{A_{n-1}} P\left(y_{n-1}, A_{n}\right) P\left(y_{n-2}, d y_{n-1}\right) \cdots P\left(x, d y_{1}\right) .
\end{aligned}
$$

Proof. (Sketch) Since $\mathbb{P}_{x}$ is defined explicitly on cylinder sets, the only fact one needs to check is that the definition of $\mathbb{P}_{x}$ is consistent, i.e., $\mathbb{P}_{x}$ on a cylinder set of length $m$ is the sum of values of $\mathbb{P}_{x}$ on cylinder subsets of length $m+1$. Then the result follows from the Kolmogorov extension theorem [Kol50] which states that 
there exists a unique probability measure on $\Omega_{x}$ extending $\mathbb{P}_{x}$ to the sigma-algebra of Borel sets.

As a corollary of Lemmas 4.11 and 4.16, we have also the following formula:

$$
\mathbb{P}_{x}\left(X_{1} \in A_{1}, \ldots, X_{n} \in A_{n}\right)=P\left(\chi_{A_{1}} P\left(\chi_{A_{2}} P\left(\cdots P\left(\chi_{A_{n-1}} P\left(\chi_{A_{n}}\right)\right) \cdots\right)\right)\right)(x) .
$$

It is useful also to mention a formula for the joint distribution of the random variables $X_{i}$ :

$$
d \mathbb{P}_{x}\left(X_{1}, \ldots, X_{n}\right)^{-1}=P\left(x, d y_{1}\right) P\left(y_{1}, d y_{2}\right) \cdots P\left(y_{n-1}, d y_{n}\right) .
$$

Lemma 4.17. The measure space $\left(\Omega_{x}, \mathbb{P}_{x}\right)$ is a standard probability measure space for $\mu$-a.e. $x \in V$.

Proof. (Sketch) To see that this property holds, we use the definition of the measure $P(x, A)$ and Assumption A. It follows that $P(x, A)>0$ if and only if $\rho_{x}\left(E_{x} \cap A\right)>0$. This means that the random variable $X_{1}$ takes values in an uncountable measure space. Clearly, the same holds for the other random variables $X_{n}$.

Remark 4.18. We remark that the symmetric measure $\rho(A \times B)=\nu(A \cap B)$ (see Example 2.16) does not satisfy the condition of Lemma4.17 because the corresponding Markov process $\left(P_{n}\right)$ is deterministic:

$$
P(x, A)=\delta_{x}(A), A \in \mathcal{B} .
$$

Hence, the corresponding operator $P$ is the identity operator.

Example 4.19 (Countable Borel equivalence relations). Suppose now that $E$ is a countable Borel equivalence relation on $(V, \mathcal{B}, \mu)$, see 2.16, part (2). Then the set $E_{x}$ of points $y$ equivalent to $x$ is countable, and the transition probabilities $P(x, y)$ are defined by the relation

$$
P(x, y)=\frac{c_{x y}}{c(x)}, \quad(x, y) \in E,
$$

where $c_{x y}$ is a symmetric function on $E$ and $c(x)=\sum_{y \in E_{x}} c_{x y}$.

We claim that a path

$$
\omega=\left(x, x_{1}, x_{2}, \ldots\right) \in \Omega_{x} \Longleftrightarrow\left(x_{i}, x_{i+1}\right) \in E_{x}, i \in \mathbb{N}_{0},
$$

where $x_{0}=x$. It follows that $\Omega_{x}=E_{x}^{\infty}$ (we assume that $c_{x y}>0, \forall y \in E_{x}$ ), and the probability measure $\mathbb{P}_{x}$ is defined on cylinder functions as follows:

$$
\mathbb{P}_{x}\left(X_{1}=y_{1}, \ldots, X_{n}=y_{n}\right)=\prod_{i=1}^{n} \frac{c_{y_{i-1} y_{i}}}{c\left(y_{i-1}\right)}, \quad y_{0}=x .
$$

We can see that, in this case, $\left(\Omega_{x}, \mathbb{P}_{x}\right)$ can be interpreted as the path space of a stationary Bratteli type diagram. All paths begin at a fixed point $x$, and the transition probability matrix $P=\left(P(x, y)_{y \in E_{x}}\right)$ is the same for all levels of this diagram. In [BJ15a], we considered the Laplace and Markov operator for arbitrary Bratteli diagrams. 
4.4. Reversible Markov processes. At the end of this section we discuss the property of reversibility for the Markov process $\left(P_{n}\right)$.

Definition 4.20. Let the objects $(V, \mathcal{B}, \mu), \rho, c(x), P$, and $R$ be as above. Suppose that $x \mapsto P(x, \cdot)$ is a measurable family of transition probabilities on the space $(V, \mathcal{B})$ which is defined by a Markov operator $P$. It is said that the corresponding Markov process is reversible if, for any sets $A, B \in \mathcal{B}$, the following relation holds:

$$
\int_{B} c(x) P(x, A) d \mu(x)=\int_{A} c(x) P(x, B) d \mu(x) .
$$

In the next result, we formulate several statements which are equivalent to reversibility of $P$.

Proposition 4.21. Let $(V, \mathcal{B}, \mu)$ be a standard measure space, $\rho=\left(x \mapsto \rho_{x}\right)$ a measure on $V \times V, \mathcal{B} \times \mathcal{B})$ such that $c(x)=\rho_{x}(V)$ is finite. Let the measure $\nu=c \mu$ and operators $R, P$ be defined as above (see [2.17, 4.1). Suppose that $P(x, \cdot)$ is the Markov process defined by the operator $P$. Then the following are equivalent:

(i) $P(x, \cdot)$ is reversible;

(ii) the Markov operator $P$ is self-adjoint in $L^{2}(\nu)$ and $\nu P=\nu$;

(iii)

$$
c(x) P(x, d y) d \mu(x)=c(y) P(y, d x) d \mu(y)
$$

(iv) the measure $\rho$ on $(V \times V, \mathcal{B} \times B)$ defined by

$$
\rho(A \times B)=\int_{V} \chi_{A} R\left(\chi_{B}\right) d \mu
$$

is symmetric where $R(f)=c P(f)$;

(v) the operator $R$ is symmetric.

Remark 4.22. If $P(x, \cdot)$ is reversible, then $P_{n}(x, \cdot)$ satisfies relation (4.11) for every $n>1$, i.e., $P_{n}(x, \cdot)$ is also reversible. This observation immediately follows from the fact that the Markov operator $P$ is self-adjoint, and therefore $P^{n}$ is also self-adjoint, see Proposition 4.21 (ii).

Proof. (i) $\Longleftrightarrow$ (ii). We first recall that $P(x, A)=P\left(\chi_{A}\right)(x)$. Then one can see that, for any sets $A, B \in \mathcal{B}_{\text {fin }}$, relation (4.11) is written as the equality of the inner products:

$$
\begin{aligned}
\left\langle\chi_{B}, P\left(\chi_{A}\right)\right\rangle_{L^{2}(\nu)} & =\int_{V} \chi_{B}(x) P\left(\chi_{A}\right)(x) c(x) d \mu(x) \\
& =\int_{V} \chi_{A}(x) P\left(\chi_{B}\right)(x) c(x) d \mu(x) \\
& =\left\langle P\left(\chi_{B}\right), \chi_{A}\right\rangle_{L^{2}(\nu)}
\end{aligned}
$$

The proof is completed by extension of the above equality by linearity to the functions from the set $\mathcal{D}_{\text {fin }}$ which is dense in $L^{2}(\nu)$ (we note that $c$ is locally integrable with resoect to $\mu$ ).

(iii) $\Longleftrightarrow$ (iv). This equivalence is obvious because the equality in (iii) means that the measure $d \rho(x, y)=d \rho_{x}(y) d \mu(x)$ is symmetric. The fact that this symmetric measure can be represented as in (iv) is proved in Proposition 2.13. 
(ii) $\Longleftrightarrow$ (iv). This results has been proved earlier. It follows immediately from Theorems 3.3 (9) and 4.4 .

(iv) $\Longleftrightarrow(\mathrm{v})$. This equivalence has been proved in Proposition 2.13

For more details regarding probability and Markov chains, the reader may consult the following items [Kol50, $\mathrm{BLP}^{+} 10$, Gei17, Ter17] and the papers cited there.

\section{Dissipation SPaCe And Stochastic analysis}

We define here a useful Hilbert space which plays an important role in the study of our finite energy space $\mathcal{H}_{E}$ in Section 6 .

Definition 5.1. On the measurable space $(\Omega, \mathcal{C})$, define a $\sigma$-finite measure $\lambda$ by

$$
\lambda:=\int_{V} \mathbb{P}_{x} d \nu(x)
$$

( $\lambda$ is infinite iff the measure $\nu$ is infinite). The Hilbert space

$$
\text { Diss }:=\left\{\frac{1}{\sqrt{2}} f: f \in L^{2}(\Omega, \lambda)\right\},
$$

is called the dissipation space.

The dissipation Hilbert space Diss is formed by functions from $L^{2}(\Omega, \lambda)$ which are rescaled by the factor $1 / \sqrt{2}$. Then

$$
\|f\|_{\mathcal{D}}=\frac{1}{\sqrt{2}}\|f\|_{L^{2}(\lambda)} .
$$

Because the partition of $\Omega$ into $\left(\Omega_{x}: x \in V\right)$ is measurable, we see that the dissipation space can be naturally decomposed into the direct integral of Hilbert spaces:

$$
L^{2}(\Omega, \lambda)=\int_{V}^{\oplus} L^{2}\left(\Omega_{x}, \mathbb{P}_{x}\right) d \nu(x)
$$

Since $x \mapsto \mathbb{P}_{x}$ is a measurable field of probability measures, we can use the following formula for integration of measurable functions $f$ over $(\Omega, \mathcal{C})$ :

$$
\lambda(f)=\int_{\Omega} f(\omega) d \lambda(\omega)=\int_{V} \mathbb{E}_{x}(f) d \nu(x)
$$

where $\mathbb{E}_{x}$ denotes the conditional expectation with respect to the measures $\mathbb{P}_{x}$,

$$
\mathbb{E}_{x}(f)=\int_{\Omega_{x}} f(\omega) d \mathbb{P}_{x}(\omega) .
$$

The inner product in the Hilbert space Diss is determined by the formula:

$$
\begin{aligned}
\langle f, g\rangle_{\mathcal{D}} & =\frac{1}{2} \int_{V} \mathbb{E}_{x}(f g) d \nu(x) \\
& =\frac{1}{2} \int_{V} \int_{\Omega_{x}} f(\omega) g(\omega) d \mathbb{P}_{x}(\omega) d \nu(x) .
\end{aligned}
$$


From the given definitions of $\mathbb{P}_{x}$ and the Markov process $\left(X_{n}\right)$, one can deduce the following formulas. We recall that $E_{n}$ denotes the conditional expectation with respect to the subalgebra $X_{n}^{-1}(\mathcal{B})$.

Lemma 5.2. Let $\left(\Omega_{x}, \mathbb{P}_{x}\right)$ be as in Section 4 , and let $P$ be the Markov operator defined in (4.1). Then

(i) $\mathbb{P}_{x} \circ X_{n}^{-1}(A)=P_{n}(x, A)=P^{n}\left(\chi_{A}\right)(x), A \in \mathcal{B}$;

(ii) $P: L^{2}\left(V, P_{n+1}(x, \cdot)\right) \rightarrow L^{2}\left(V, P_{n}(x, \cdot)\right)$ is contractive for all $n$;

(iii) $P(f) \circ X_{n}=\mathbb{E}\left[f \circ X_{n+1} \mid \mathcal{F}_{n}\right]=E_{n}\left(f \circ X_{n+1}\right)$.

Proof. (i) This formula follows from the definition of the measure $\mathbb{P}_{x}$, see (4.8) and (4.9).

(ii) Let $f \in L^{2}\left(V, P_{n+1}(x, \cdot)\right)$. Then

$$
\begin{aligned}
\|P(f)\|_{P_{n}}^{2} & =\int_{V} P(f)^{2}(y) P_{n}(x, d y) \\
& \leq \int_{V} P\left(f^{2}\right)(y) P_{n}(x, d y) \\
& =\int_{V} f^{2}(y) P_{n+1}(x, d y) \\
& =\|f\|_{P_{n+1}}^{2} .
\end{aligned}
$$

(iii) In fact, we will prove a slightly more general result: for any Borel functions $f, h$, one has

$$
\mathbb{E}_{x}\left[\left(h \circ X_{n}\right)\left(f \circ X_{n+1}\right)\right]=\mathbb{E}_{x}\left[\left(h \circ X_{n}\right)\left(P(f) \circ X_{n}\right)\right]
$$

Indeed, we use (i) to show that

$$
\begin{aligned}
\mathbb{E}_{x}\left[\left(h \circ X_{n}\right)\left(P(f) \circ X_{n}\right)\right] & =\int_{V} h(y) P(f)(y) P_{n}(x, d y) \\
& =\iint_{V \times V} h(y) f(z) P(y, d z) P_{n}(x, d y) \\
& =\mathbb{E}_{x}\left[\left(h \circ X_{n}\right)\left(f \circ X_{n+1}\right)\right] .
\end{aligned}
$$

We now show that $L^{2}(\nu)$ is isometrically embedded into Diss. Let $W_{n}$ be defined by the relation

$$
W_{n}(f)=\sqrt{2}\left(f \circ X_{n}\right), \quad n \in \mathbb{N}
$$

Lemma 5.3. The operator $W_{n}$ is an isometry from $L^{2}(\nu)$ to Diss for any $n \in \mathbb{N}$. 
Proof. We compute

$$
\begin{aligned}
\left\|W_{n}(f)\right\|_{\text {Diss }}^{2} & =\int_{V} \mathbb{E}_{x}\left(f \circ X_{n}(\omega)^{2}\right) d \nu(x) \\
& \left.=\int_{V} \int_{\Omega_{x}} f\left(X_{n}(\omega)\right)^{2}\right) d \mathbb{P}_{x}(\omega) d \nu(x) \\
& =\int_{V} \int_{V} f(y)^{2} d P_{n}(x, d y) d \nu(x) \\
& \left.=\int_{V} \int_{V} f(y)^{2} d \rho(x, y) \quad \text { ( } \rho \text { is symmetric }\right) \\
& =\int_{V} \int_{V} f(x)^{2} d \rho(x, y) \\
& =\int_{V} f(x)^{2} c(x) d \mu(x) \\
& =\|f\|_{L^{2}(\nu)}^{2} .
\end{aligned}
$$

We will use the isometry $W_{n}$ defined in (5.5) in order to extend the definition of the operator $P$ to the dissipation space. This approach is described in the following steps.

Let $F_{n}$ be a function of $n+1$ variables,

$$
F_{n}: \underbrace{V \times \cdots \times V:}_{n+1 \text { times }} \rightarrow \mathbb{R}, n \in \mathbb{N}
$$

Set

$$
\Phi_{n}(\omega):=F\left(X_{0}(\omega), \ldots X_{n}(\omega)\right)
$$

Clearly, the Hilbert space Diss contains a dense subset which is constituted by functions of the form $\Phi_{n}, n \in \mathbb{N}$.

Define abounded linear operator $S$ acting in Diss. It suffices to define it on functions $\Phi_{n}$ :

$$
\left(S \Phi_{n}\right)(\omega)=\int_{V} F\left(X_{0}(\omega), \ldots X_{n-1}(\omega), y\right) P\left(X_{n-1}(\omega), d y\right)
$$

Lemma 5.4. The operator $S$ defined by (5.6) is contractive and self-adjoint. 
Proof. To see that $S$ is contractive as an operator on Diss, we use (4.10) in the following computation:

$$
\begin{aligned}
& \left\|(S F)\left(X_{0}, \ldots, X_{n}\right)\right\|_{\mathcal{D}}^{2} \\
& =\frac{1}{2} \int_{V} \mathbb{E}_{x}\left(\int_{V} F\left(X_{0}, \ldots, X_{n-1}, y\right) P\left(X_{n-1}, d y\right)\right)^{2} d \nu(x) \\
& \leq \frac{1}{2} \int_{V} \mathbb{E}_{x}\left(\int_{V} F^{2}\left(X_{0}, \ldots X_{n-1}, y\right) P\left(X_{n-1}, d y\right)\right) d \nu(x) \\
& =\frac{1}{2} \iiint F^{2}\left(X_{0}(\omega), \ldots X_{n-1}(\omega), y\right) P\left(X_{n-1}(\omega), d y\right) d \mathbb{P}_{x}(\omega) d \nu(x) \\
& =\frac{1}{2} \int \ldots \int F^{2}\left(x, x_{1}, \ldots, y\right) P\left(x, d x_{1}\right) \cdots P\left(x_{n-2}, d x_{n-1}\right) P\left(x_{n-1}, d y\right) d \nu(x) \\
& =\frac{1}{2} \int_{V} \int_{\Omega_{x}} F^{2}\left(X_{0}(\omega), \ldots X_{n}(\omega)\right) d \mathbb{P}_{x}(\omega) d \nu(x) \\
& =\|F\|_{\mathcal{D}}^{2}
\end{aligned}
$$

Using similar argument and the invariance of $\nu$ with respect to $P$, we can show that, for any cylinder functions $F, G$,

$$
\langle S F, G\rangle_{\mathcal{D}}=\langle F, S G\rangle_{\mathcal{D}}
$$

i.e., $S$ is self-adjoint in Diss.

Remark 5.5. Suppose that $\Phi(\omega)=F \circ X_{n}(\omega)$ for some $n$, where $F$ is a function from $L^{2}(\nu)$. Then we can deduce that

$$
S\left(F \circ X_{n}\right)=P(F) \circ X_{n}
$$

This means that the following diagram commutes:

$$
\begin{array}{ccc}
L^{2}(\nu) & \stackrel{P}{\longrightarrow} & L^{2}(\nu) \\
\downarrow W_{n} & & \downarrow W_{n} \\
\text { Diss } & \stackrel{S}{\longrightarrow} & \text { Diss }
\end{array}
$$

In the next two results, we discuss the orthogonality properties in the dissipation space $L^{2}(\Omega, \lambda)$.

Lemma 5.6 (Key lemma). Let $g_{1}, g_{2}$ be functions from $L^{2}(\nu)$. Then

$$
\left\langle g_{1} \circ X_{n}, P\left(g_{2}\right) \circ X_{n}-g_{2} \circ X_{n+1}\right\rangle_{\mathcal{D}}=0 .
$$

Proof. It follows from (5.4) that the result would follow if we proved that the functions $g_{1} \circ X_{n}$ and $P\left(g_{2}\right) \circ X_{n}-g_{2} \circ X_{n+1}$ are orthogonal in $L^{2}\left(\Omega_{x}, \mathbb{P}_{x}\right)$ for a.e. 
$x$. We use here Lemma 5.2 and (4.7) to compute the inner product:

$$
\begin{aligned}
& \left\langle g_{1} \circ X_{n}, P\left(g_{2}\right) \circ X_{n}-g_{2} \circ X_{n+1}\right\rangle_{\mathbb{P}_{x}} \\
= & \mathbb{E}_{x}\left(E_{n}\left(g_{1} \circ X_{n}\right)\left(P\left(g_{2}\right) \circ X_{n}-g_{2} \circ X_{n+1}\right)\right) \\
= & \mathbb{E}_{x}\left(\left(g_{1} \circ X_{n}\right) E_{n}\left(P\left(g_{2}\right) \circ X_{n}-g_{2} \circ X_{n+1}\right)\right) \\
= & \mathbb{E}_{x}\left(\left(g_{1} \circ X_{n}\right)\left(P\left(g_{2}\right) \circ X_{n}-E_{n}\left(g_{2} \circ X_{n+1}\right)\right)\right) \\
= & \mathbb{E}_{x}\left(\left(g_{1} \circ X_{n}\right)\left(P\left(g_{2}\right) \circ X_{n}-P\left(g_{2}\right) \circ X_{n}\right)\right) \\
= & 0
\end{aligned}
$$

Proposition 5.7. For any function $f \in L^{2}(\nu)$ and any $n \in \mathbb{N}$,

$$
(I-P)(f) \circ X_{n} \perp\left(P(f) \circ X_{n}-f \circ X_{n+1}\right)
$$

in the dissipation space Diss.

Proof. To prove (5.8), it suffices to show that

$$
\left(f \circ X_{n}\right) \perp\left(P(f) \circ X_{n}-f \circ X_{n+1}\right)
$$

and

$$
P(f) \circ X_{n} \perp\left(P(f) \circ X_{n}-f \circ X_{n+1}\right)
$$

Relation (5.9) has been proved in the Key Lemma (Lemma 5.6). It follows from Lemma 5.2 (iii) and the proof of Key Lemma that, for $\nu$-a.e. $x \in V$,

$$
\begin{aligned}
& \mathbb{E}_{x}\left(\left(P(f) \circ X_{n}\right)\left(P(f) \circ X_{n}-f \circ X_{n+1}\right)\right) \\
= & \mathbb{E}_{x}\left(\left(P(f) \circ X_{n}\right) E_{n}\left(P(f) \circ X_{n}-f \circ X_{n+1}\right)\right) \\
= & \mathbb{E}_{x}\left(\left(P(f) \circ X_{n}\right)\left(P(f) \circ X_{n}-E_{n}\left(f \circ X_{n+1}\right)\right)\right) \\
= & 0 .
\end{aligned}
$$

This proves (5.10) and we are done.

We return to the properties of the dissipation space Diss. Denote by $\sigma$ the shift in the space $(\Omega, \lambda)$, i.e.,

$$
\sigma: \omega=\left(\omega_{0}, \omega_{1}, \ldots\right) \mapsto\left(\omega_{1}, \omega_{2}, \ldots\right) .
$$

Equivalently, $\sigma$ is determined by the relation $X_{n} \circ \sigma=X_{n+1}$. Clearly, $\sigma$ is a measurable endomorphism of $(\Omega, \lambda)$, and $\sigma$ acts on the measure $\lambda$ by the formula

$$
\lambda \circ \sigma^{-1}(\psi)=\int_{V} \mathbb{P}_{x}(\psi \circ \sigma) d \nu(x), \quad \psi \in \mathcal{F}(\Omega, \mathcal{C}) .
$$

Lemma 5.8. (1) Denote by $L=L_{\sigma}$ the operator on $L^{2}(\lambda)$ acting as follows:

$$
L(f)=f \circ \sigma, \quad f \in L^{2}(\lambda) .
$$

Then $L$ is an isometry.

(2) Condition $\nu P=\nu$ implies that $\lambda \circ \sigma^{-1}=\lambda$. If $d(\nu P)(x)=m(x) d \nu$, then

$$
\frac{d \lambda \circ \sigma^{-1}}{d \lambda}=\frac{1}{m \circ X_{0}} \text {. }
$$


Proof. We leave the proof to the reader.

Remark 5.9. (1) To emphasize the fact that the measure $\lambda$ is defined by the operator $P$, we will use also the notation $\lambda_{P}$. We note that $\lambda$ is not, in general, a probability measure, so that we cannot use the language of probability theory considering the Markov process on $(\Omega, \mathcal{C}, \lambda)$. If one took a probability measure on $(V, \mathcal{B})$ equivalent to $\nu$ (and $\mu$ ), then $\lambda$ would be a probability measure. But it is important to mention that, for transient Markov processes, the measure $\lambda_{P}$ must be infinite.

(2) We use the measure $\nu($ not $\mu$ ) in the definition of $\lambda$ and in (5.2). The reason for this is based on the fact that $\nu$ is invariant with respect to $P$.

Proposition 5.10. Let $A$ and $B$ be any two sets from $\mathcal{B}_{\text {fin }}$. Then

$$
\rho_{n}(A \times B)=\lambda\left(X_{0} \in A, X_{n} \in B\right), \quad n \in \mathbb{N} .
$$

In other words, this equality can be interpreted in the following way: for the Markov process $\left(P_{n}\right)$, the "probability" to get in $B$ for $n$ steps starting somewhere in $A$ is exactly $\rho_{n}(A \times B)>0$.

We recall that the measures $\lambda$ and $\rho$ are, in general, not probability.

Proof. It follows from the definition of $\lambda$ and $\rho$ that

$$
\begin{aligned}
\lambda\left(X_{0} \in A, X_{n} \in B\right) & =\int_{A} \mathbb{P}_{x}\left(X_{n} \in B\right) d \nu(x) \\
& =\int_{A} P_{n}(x, B) d \nu(x) \\
& =\int_{A} P^{n}\left(\chi_{B}\right)(x) d \nu(x) \\
& =\int_{V} \chi_{A}(x) P^{n}\left(\chi_{B}\right)(x) d \nu(x) \\
& =\rho_{n}(A \times B)
\end{aligned}
$$

The following result is proved analogously to Proposition 5.10, We leave the details to the reader.

Corollary 5.11. The Markov process $\left(P_{n}\right)$ is irreducible, i.e., for any sets $A, B \in$ $\mathcal{B}_{\text {fin }}$ there exists some $n$ such that $\left\langle\chi_{A}, P^{n}\left(\chi_{B}\right)\right\rangle_{L^{2}(\nu)}>0$.

\section{Finite energy space: Decompositions and covariance computation}

This section is focused on a measurable analogue of the finite energy space that has been extensively studied in the special case of weighted networks. Before formulating our main definitions and results, we discuss a construction of a "connected graph" on the set $\mathcal{B}_{\text {fin }}$. 
6.1. $\mathcal{B}_{\text {fin }}$ as a connected graph. Let $(V, \mathcal{B}, \mu)$ be a standard measure space, and let $\rho$ be a symmetric measure on $(V \times V, \mathcal{B} \times \mathcal{B})$.

Lemma 6.1. Suppose that $c(x) \in L_{\text {loc }}^{1}(\mu)$. Then, for any set $A \in \mathcal{B}_{\text {fin }}$,

$$
\rho\left(A \times A^{c}\right)<\infty
$$

where $A^{c}=V \backslash A$. The converse is not true, in general.

Proof. The following computation uses the definition of $\rho$ and local integrability of the function $c$ :

$$
\begin{aligned}
\rho\left(A \times A^{c}\right) & =\int_{V} \chi_{A}(x) R\left(\chi_{A^{c}}\right)(x) d \mu(x) \\
& =\int_{A}\left(\int_{V} \chi_{A^{c}} d \rho_{x}\right) d \mu(x) \\
& =\int_{A} \rho_{x}\left(A^{c}\right) d \mu(x) \\
& \leq \int_{A} c(x) d \mu(x) \\
& <\infty
\end{aligned}
$$

The converse statement is false because if $c \in L^{1}(\mu)$, then $\rho\left(A \times A^{c}\right)<\infty$ does not imply that $A \in \mathcal{B}_{\text {fin }}$.

The following definition introduces "edges" on the set $\mathcal{B}_{\text {fin }}$.

Definition 6.2. For a symmetric measure $\rho$ on $(V \times V, \mathcal{B} \times B)$, we say that two sets $A$ and $B$ from $\mathcal{B}_{\text {fin }}$ are connected by an edge $e$ if $\rho(A \times B)>0$. Then $\alpha$ : $(A, B) \rightarrow \rho(A \times B)$ is a symmetric function defined on the set of edges in $\mathcal{B}_{\text {fin }}$.

Proposition 6.3. Let $(V, \mathcal{B}, \mu)$ and $\rho$ be as above. Then any two sets $A$ and $B$ from $\mathcal{B}_{\text {fin }}$ are connected in $\mathcal{B}_{\text {fin }}$ by a finite path.

Proof. We will show that there exists a finite sequence $\left(A_{i}: 0 \leq i \leq n\right)$ of disjoint subsets from $\mathcal{B}_{\text {fin }}$ such that $A_{0}=A, \rho\left(A_{i} \times A_{i+1}\right)>0$, and $\rho\left(A_{n} \times B\right)>0$, $i=0, \ldots, n-1$.

If $\rho(A \times B)>0$, then nothing to prove, so that we can assume that $\rho(A \times B)=0$.

Let $\xi=\left(C_{i}: i \in \mathbb{N}\right)$ be a partition of $V$ into disjoint subsets of positive finite measure such that $C_{i} \in \mathcal{B}_{\text {fin }}$ for all $i$. Without loss of generality, we can assume that the sets $A$ and $B$ are included in $\xi$. Let for definiteness, $A=C_{0}$.

Since $\rho\left(A \times A^{c}\right)>0$ (by Lemma 6.1), there exists a set $C_{i_{1}} \in \xi$ such that $\rho\left(A \times C_{i_{1}}\right)>0$ and $\rho\left(A \times C_{j}\right)=0$ for all $0<j<i_{1}$. Set

$$
A_{1}:=\bigcup_{0<j \leq i_{1}} C_{j}
$$

It is clear that $A_{1} \in \mathcal{B}_{\text {fin }}$ and $\rho\left(A_{0} \times A_{1}\right)>0$. If $\rho\left(A_{1} \times B\right)>0$, then we are done. If not, we proceed as follows. Because of the property $\rho\left(A_{1} \times A_{1}^{c}\right)>0$, there exists 
some $i_{2}>i_{1}$ such that $\rho\left(A_{1} \times C_{i_{2}}\right)>0$ and $\rho\left(A_{1} \times C_{j}\right)=0$ for all $i_{1}<j<i_{2}$. Set

$$
A_{2}:=\bigcup_{i_{1} \leq j \leq i_{2}} C_{j} .
$$

Then $\rho\left(A_{1} \times A_{2}\right)>0$, and we check whether $\rho\left(A_{2} \times B\right)>0$. If not, we continue in the same manner by constructing consequently disjoint sets $A_{i}$ satisfying the property $\rho\left(A_{i} \times A_{i+1}\right)>0$. Since $B$ is an element of $\xi$, this process will terminate. This means that there exists some $n$ such that $A_{n} \supset B$. This argument proves the proposition.

It follows from Proposition 5.10 and Proposition 6.3 that, for the corresponding Markov process, the assumed positive probability to get from $A$ to $B$ can be interpreted as the connectedness of $\mathcal{B}_{\text {fin }}$. This property is formulated in the following assertion.

Corollary 6.4. Let $P(x, \cdot)$ be the Markov process defined in Section 4. For any two sets $A$ and $B$ from $\mathcal{B}_{\text {fin }}$ and $x \in A$,

$$
\mathbb{P}_{x}\left(X_{1} \in A_{1}, \ldots, X_{n} \in B\right)>0
$$

if and only if there exists a chain of sets $A=A_{0}, A_{1}, \ldots, A_{n}=B$ such that $\rho\left(A_{i-1} \times\right.$ $\left.A_{i}\right)>0, i=1, \ldots, n$.

Remark 6.5. Suppose that $\left(\xi_{n}\right), \xi_{n}=\left(A_{i}^{(n)}: n \in \mathbb{N}\right)$ is a sequence of countable partitions of $(V, \mathcal{B}, \mu)$ that satisfies the properties:

(i) $\xi_{n+1}$ refines $\xi_{n}$ : every $A_{i}^{(n)}$ is a $\xi_{n+1}$-set, i.e., it is a union of some elements of $\xi_{n+1}$,

(ii) the $\sigma$-algebra generated by $\xi_{n}$-sets is $\mathcal{B}$.

Property (ii) is equivalent to the fact that, for every point $x$, there exists a nested sequence $\left(A_{\left.i_{n}(x)\right)}^{(n)}\right)$ such that

$$
\bigcap_{n \in \mathbb{N}} A_{\left.i_{n}(x)\right)}^{(n)}=\{x\}
$$

In other words, this means that we assign, for every point $x \in V$, an infinite word over a sequence of countable alphabets determined by atoms of partitions $\xi_{n}$.

Denote by $c_{i, j}^{(n)}=\rho\left(A_{i}^{(n)} \times A_{j}^{(n)}\right)$ and set

$$
c_{i_{n}(x)}^{(n)}=\sum_{j \sim i_{n}(x)} c_{i_{n}(x), j}^{(n)}
$$

where $j \sim i$ means that $\rho\left(A_{i} \times A_{j}\right)>0$.

It can be proved that

Claim. (1) $c_{i_{n}(x)}^{(n)}<\infty$ for every $i, j$.

(2) $c_{i_{n}(x)}^{(n)} \geq c_{i_{n+1}(x)}^{(n+1)}$. 
Hence, we can define the function $c(x)$ by setting

$$
c(x)=\lim _{n \rightarrow \infty} c_{i_{n}(x)}^{(n)} .
$$

6.2. Definition and properties of the finite energy space $\mathcal{H}_{E}$. We consider a class of Borel functions over $(V, \mathcal{B}, \mu)$ which is formed by functions of finite energy. In other words, this section is focused on a measurable analogue of the energy Hilbert space which was extensively studied in the context of discrete networks, see e.g. Cho14, JP16, Jor12, LP16].

Definition 6.6. Let $(V, \mathcal{B}, \mu)$ be a standard measure space with $\sigma$-finite measure $\mu$. Suppose that $\rho$ is a symmetric measure on the Cartesian product $(V \times V, \mathcal{B} \times \mathcal{B})$. We say that a Borel function $f: V \rightarrow \mathbb{R}$ belongs to the finite energy space $\mathcal{H}_{E}=\mathcal{H}$ if

$$
\iint_{V \times V}(f(x)-f(y))^{2} d \rho(x, y)<\infty .
$$

If the measure $\rho$ is defined in terms of a conductance function $c_{x y}$, then a function $f$ is in $\mathcal{H}$ when

$$
\int_{V}\left(\int_{V} c_{x y}(f(x)-f(y))^{2} d \mu(y)\right) d \mu(x)<\infty
$$

Remark 6.7. (1) It follows from the Cauchy-Schwarz inequality in the space $L^{2}(\rho)$ that the set $\mathcal{H}$ is a vector space. It contains all constant function $k$. Since for the functions $f$ and $f+k$, the quantity in (6.2) is the same, we can identify such functions in the space $\mathcal{H}$. That is $\mathcal{H}$ can be treated as the space of classes of equivalent functions where $f \sim g$ iff $f-g$ is a constant. With some abuse of notation we will denote this quotient space again by $\mathcal{H}$. We show below that $\mathcal{H}$ is a Hilbert space.

(2) Definition 6.6 assumes that a symmetric irreducible measure $\rho$ is fixed on $(V \times V, \mathcal{B} \times \mathcal{B})$. This means that the space of functions $f$ on $(V, \mathcal{B})$ satisfying (6.2) depends on $\rho$ and must be written as $\mathcal{H}_{E}(\rho)$. Since we do not study the dependence of $\mathcal{H}_{E}(\rho)$ of $\rho$, we will write $\mathcal{H}_{E}$ or even $\mathcal{H}$ below.

In other words, we can define a bilinear form $\xi(f, g)$ in the space $\mathcal{H}$ by the formula

$$
\xi(f, g):=\frac{1}{2} \iint_{V \times V}(f(x)-f(y))(g(x)-g(y)) d \rho(x, y) .
$$

We set $\xi(f)=\xi(f, f)$. The domain of $\xi$ is the set of function $f$ such that $\xi(f)<\infty$, and, assuming connectedness, the kernel of $\xi$ is $\mathbb{R} \mathbf{1}$. Then we see that the space $\mathcal{H}$ defined above coincides with $\operatorname{dom}(\xi) / \operatorname{ker}(\xi)$.

Setting $\langle f, g\rangle_{\mathcal{H}}=\xi(f, g)$, we define an inner product on $\mathcal{H}$. Then

$$
\|f\|_{\mathcal{H}}^{2}:=\frac{1}{2} \iint_{V \times V}(f(x)-f(y))^{2} d \rho(x, y), \quad f \in \mathcal{H},
$$

turns $\mathcal{H}$ in a normed vector space. 
Lemma 6.8. The map

$$
\partial: f(x) \mapsto F_{f}(x, y):=\frac{1}{\sqrt{2}}(f(x)-f(y))
$$

is an isometric embedding of the space $\mathcal{H}$ into $L^{2}(\rho)$.

Proof. This fact follows immediately from Definition 6.6 and (6.5).

Theorem 6.9. $\left(\mathcal{H},\|\cdot\|_{\mathcal{H}}\right)$ is a Hilbert space.

Proof. We need to check that the normed space $\left(\mathcal{H},\|\cdot\|_{\mathcal{H}}\right)$ is complete.

Suppose $\left(f_{n}\right)$ is a Cauchy sequence in $\mathcal{H}$. Then, by Lemma 6.8, the sequence $\left(F_{n}\right)$, where $F_{n}(x, y):=f_{n}(x)-f_{n}(y)$, is Cauchy in $L^{2}(\rho)$. Hence, there exists a function $G(x, y)$ such that

$$
\left\|F_{n}-G\right\|_{L^{2}(\rho)} \rightarrow 0, \quad n \rightarrow \infty .
$$

There exists a subsequence $\left(F_{n_{k}}\right)$ that converges pointwise to $G$ for all $(x, y) \notin N$ where $\rho(N)=0$. Let $N^{y}:=\{x:(x, y) \in N\}$.

Then, for $\mu$-a.e. $y \in V$, we have $\rho_{x}\left(N^{y}\right)=0$. In particular, this means that there exists some $y_{0}$ such that

$$
F_{n_{k}}\left(x, y_{0}\right) \rightarrow G\left(x, y_{0}\right), \quad \rho_{x^{-} \text {a.e. }}
$$

Therefore the function $g(x):=G\left(x, y_{0}\right)$ is correctly defined.

It remains to prove that $G(x, y)=g(x)-g(y)$. Indeed, for $(x, y) \notin N$,

$$
\begin{aligned}
G(x, y) & =\lim _{k \rightarrow \infty}\left(f_{n_{k}}(x)-f_{n_{k}}(y)\right) \\
& =\lim _{k \rightarrow \infty}\left(f_{n_{k}}(x)-f_{n_{k}}\left(y_{0}\right)\right)-\lim _{k \rightarrow \infty}\left(f_{n_{k}}(y)-f_{n_{k}}\left(y_{0}\right)\right) \\
& =g(x)-g(y) .
\end{aligned}
$$

In other words, we have proved that the Cauchy sequence $\left(f_{n}\right)$ converges to $g(x)$ in $\mathcal{H}$.

Theorem 6.10. Suppose that $\rho$ is a symmetric irreducible measure on $(V \times V, \mathcal{B} \times$ $\mathcal{B})$, and $\mathcal{H}_{E}=\mathcal{H}_{E}(\rho)$ is the finite energy space. If $f \in \mathcal{H}_{E}$ is a function such that $\|f\|_{\mathcal{H}_{E}}=0$, then $f(x)$ is a constant $\mu$-a.e.

Proof. We can interpret the set $\Omega_{x}$ as the set of all paths which begin at $x$ and are determined by the Markov process $P(x, \cdot)$. Then we immediately deduce from Corollary 6.13 that the function $f$ with the property $\|f\|_{\mathcal{H}}=0$ is constant along any such path.

Suppose, for contrary, that $f(x)$ is not constant on $(V, \mathcal{B}, \mu)$. Then there exists some $a \in \mathbb{R}$ such that the sets $A:=\{f<a\}$ and $B:=\{f>a\}$ both have positive measure $\mu$. Take subsets $A_{0} \subset A$ and $B_{0} \subset B$ of finite positive measure. By connectedness of $\mathcal{B}_{\text {fin }}$, there exists a set of positive measure $A^{\prime} \subset A_{0}$ such that any path beginning in $A^{\prime}$ gets in $B_{0}$ in finitely many iterations. We obtain a contradiction. 
6.3. Energy space is embedded into dissipation space. Let $P$ be a Markov operator and $x$ is a fixed point in $V$. Denote by $P(x, A)$ the probability measure defined by $P$ as in Section 4 . This means that

$$
P(x, f)=\int_{V} f(y) d \bar{\rho}_{x}(y)=P(f)(x)
$$

where $f$ is a Borel function. If $X_{n}(\omega)$ is a corresponding sequence of random variables on $\Omega_{x}$, then we have the following formulas for the conditional expectation $\mathbb{E}_{x}$ with respect to the probability measure $\mathbb{P}_{x}$ :

$$
\begin{array}{r}
\mathbb{E}_{x}\left(f \circ X_{0}\right)=\int_{\Omega_{x}} f\left(X_{0}(\omega)\right) d \mathbb{P}_{x}(\omega)=\int_{\Omega_{x}} f(x) d \mathbb{P}_{x}(\omega)=f(x), \\
\mathbb{E}_{x}\left(f \circ X_{1}\right)=\int_{\Omega_{x}} f\left(X_{1}(\omega)\right) d \mathbb{P}_{x}(\omega)=\int_{V} f(y) P(x, d y)=P(f)(x)
\end{array}
$$

where $y=X_{1}(\omega)$.

Definition 6.11. Define a linear operator $\partial: \mathcal{H}_{E} \rightarrow$ Diss by the formula:

$$
\partial: f \mapsto f \circ X_{1}-f \circ X_{0} .
$$

Similarly, we set

$$
\partial_{n}: f \mapsto f \circ X_{n+1}-f \circ X_{n} .
$$

Remark that we use the same notation $\partial$ as in (6.6) of Lemma 6.8 because these operators are essentially similar.

Lemma 6.12. The operator $\partial: \mathcal{H}_{E} \rightarrow$ Diss defined in (6.9) is an isometry.

Proof. Let $f \in \mathcal{H}_{E}$. We use the definition of the norm in Diss and in the energy space $\mathcal{H}_{E}$ :

$$
\begin{aligned}
\|\partial f\|_{\mathcal{D}}^{2} & =\frac{1}{2} \int_{V} \mathbb{E}_{x}\left[\left(f \circ X_{0}-f \circ X_{1}\right)^{2}\right] d \nu(x) \\
& =\frac{1}{2} \int_{V}(f(x)-f(y))^{2} P(x, d y) d \nu(x) \\
& =\frac{1}{2} \int_{V}(f(x)-f(y))^{2} d \rho_{x}(y) d \mu(x) \\
& =\frac{1}{2} \int_{V}(f(x)-f(y))^{2} d \rho(x, y) \\
& =\|f\|_{\mathcal{H}_{E}}^{2} .
\end{aligned}
$$

As a corollary, we have the following formula that is used below.

Corollary 6.13. For $f \in \mathcal{H}$ and $\nu=c \mu$, we have

$$
\|f\|_{\mathcal{H}_{E}}^{2}=\frac{1}{2} \int_{V} \mathbb{E}_{x}\left[\left(f \circ X_{1}-f \circ X_{0}\right)^{2}\right] d \nu(x) .
$$

Proof. See the proof of Lemma 6.12, 
In the next statements we strengthen the result of Corollary 6.13 using the orthogonal decomposition given in Proposition 5.7.

Theorem 6.14. Let $f \in \mathcal{H}_{E}$. Then

$$
\begin{aligned}
\|f\|_{\mathcal{H}_{E}}^{2} & =\frac{1}{2}\left(\int_{V}\left(P\left(f^{2}\right)-P(f)^{2}\right) d \nu+\int_{V}(P(f)-f)^{2} d \nu\right) \\
& =\frac{1}{2}\left(\int_{V}\left(P\left(f^{2}\right)-P(f)^{2}\right) d \nu+\|f-P(f)\|_{L^{2}(\nu)}^{2}\right) .
\end{aligned}
$$

In particular, both integrals in the right hand side of (6.11) are finite and nonnegative. Moreover, $\operatorname{Var}_{x}\left(f \circ X_{1}\right)=P\left(f^{2}\right)-P(f)^{2} \geq 0$ and $\operatorname{Var}_{x}\left(f \circ X_{1}\right) \in L^{1}(\nu)$, for any $f \in \mathcal{H}_{E}$.

Proof. By Lemma 6.12, it suffices to prove that the right hand side of (6.11) equals $\|\partial f\|_{\mathcal{D}}^{2}$. Indeed, we can use the orthogonal decomposition given in Proposition 5.7 and write

$$
\|\partial f\|_{\mathcal{D}}^{2}=\left\|f \circ X_{0}-P(f) \circ X_{0}\right\|_{\mathcal{D}}^{2}+\left\|P(f) \circ X_{0}-f \circ X_{1}\right\|_{\mathcal{D}}^{2} .
$$

In the proof below we use the following equality:

$$
\begin{aligned}
& \operatorname{Var}_{x}\left(f \circ X_{1}\right) \\
= & \int_{V}(P(f)(x)-f(y))^{2} P(x, d y) \\
= & P(f)^{2}(x)-2 P(f)(x) \int_{V} f(y) P(x, d y)+\int_{V} f(y)^{2} P(x, d y) \\
= & P(f)^{2}(x)-2 P(f)^{2}(x)+P\left(f^{2}\right)(x) \\
= & P\left(f^{2}\right)(x)-P(f)^{2}(x) .
\end{aligned}
$$

Then the computation of $\|\partial f\|_{\mathcal{D}}^{2}$ goes as follows:

$$
\begin{aligned}
\|\partial f\|_{\mathcal{D}}^{2}= & \frac{1}{2} \int_{V} \mathbb{E}_{x}\left[(I-P)(f)^{2} \circ X_{0}\right] d \nu(x) \\
& +\frac{1}{2} \int_{V} \mathbb{E}_{x}\left[\left(P(f) \circ X_{0}-f \circ X_{1}\right)^{2}\right] d \nu(x) \\
= & \frac{1}{2} \int_{V}(f-P(f))^{2}(x) P(x, d y) d \nu(x) \\
& +\frac{1}{2} \int_{V}(P(f)(x)-f(y))^{2} P(x, d y) d \nu(x) \\
= & \frac{1}{2} \int_{V}(f-P(f))^{2}(x) d \nu(x) \\
& +\frac{1}{2} \int_{V}\left(P\left(f^{2}\right)(x)-P(f)^{2}(x)\right) d \nu(x) .
\end{aligned}
$$

The proof is complete.

Theorem 6.14 allows us to deduce a number of important corollaries. 
Corollary 6.15. (1) If $f \in \mathcal{H}_{E}$, then $f-P(f) \in L^{2}(\nu)$ and $P\left(f^{2}\right)-P(f)^{2} \in L^{1}(\nu)$. The operator

$$
I-P: f \mapsto f-P(f): \mathcal{H}_{E} \rightarrow L^{2}(\nu)
$$

is contractive, i.e., $\|I-P\|_{\mathcal{H}_{E} \rightarrow L^{2}(\nu)} \leq 1$.

$$
\begin{aligned}
\|f\|_{\mathcal{H}_{E}}=0 & \Longleftrightarrow\left\{\begin{array}{c}
P\left(f^{2}\right)=P(f)^{2} \\
P(f)=f
\end{array}\right. \\
& \Longleftrightarrow \text { both } f \text { and } f^{2} \text { are harmonic functions. }
\end{aligned}
$$

(3) Let $f \in \mathcal{H}_{E}$, then

$$
\begin{aligned}
f \in \mathcal{H a r m} & \Longleftrightarrow\|f\|_{\mathcal{H}_{E}}^{2}=\frac{1}{2} \int_{V}\left(P\left(f^{2}\right)(x)-(P f)^{2}(x)\right) d \nu(x) \\
& \Longleftrightarrow\|f\|_{\mathcal{H}_{E}}^{2}=\frac{1}{2} \int_{V} \operatorname{Var}_{x}\left(f \circ X_{1}\right) d \nu(x) .
\end{aligned}
$$

Proof. Statement (1) immediately follows from (6.11).

To see that (2) holds we use again (6.11). The right hand side is zero if and only if $P(f)=f$ and $P\left(f^{2}\right)=P(f)^{2}$ a.e. (recall that, for any function $\left.f, P\left(f^{2}\right) \geq P(f)^{2}\right)$. Since $f$ is harmonic, the latter means that $f^{2}$ is harmonic.

(3) This observation is a consequence of (6.11), Theorem 6.14.

We remark that formula (6.11) for the norm $\|f\|_{\mathcal{H}_{E}}^{2}$ consists of two terms: the deterministic term is $\|f-P(f)\|_{L^{2}(\nu)}^{2}$ and the stochastic term is $\int_{V}\left(P\left(f^{2}\right)-P(f)^{2}\right) d \nu$. Thus, the norm of a harmonic function is completely determined by the stochastic term.

It can be shown, using Theorem 6.14, that the following result holds. We leave its proof for the reader.

\section{Corollary 6.16.}

$$
\int_{V} \operatorname{Var}_{x}\left(f \circ X_{1}\right) d \nu(x)=\int_{V} \operatorname{Var}_{x}\left(f \circ X_{n}\right) d \nu(x), \quad n \in N .
$$

In what follows we will deal with the so called Riesz decomposition of functions from the energy space $\mathcal{H}_{E}$. It is important to note, that in this case, we make an additional assumption about the Markov process $\left(P_{n}\right)$ : it must be transient.

Corollary 6.17. Assume that $\left(P_{n}\right)$ is a transient Markov process, i.e., Green's function

$$
G(x, A):=\sum_{n \in \mathbb{N}_{0}} P_{n}(x, A)
$$

is a.e. finite for every $A \in \mathcal{B}$. Then every function $f \in \mathcal{H}_{E}$ has a unique decomposition (Riesz decomposition) $f=G(\varphi)+h$ where $\varphi \in L^{2}(\nu)$ and $h \in \mathcal{H a r m}_{E}$.

Moreover, for every $f \in \mathcal{H}_{E}$,

$$
\|f\|_{\mathcal{H}_{E}}^{2}=\frac{1}{2}\left(\|\varphi\|_{L^{2}(\nu)}^{2}+\int_{V}\left(P\left(h^{2}\right)-h^{2}\right) d \nu\right) .
$$


Proof. (Sketch) Let $f \in \mathcal{H}_{E}$, then $\varphi=(I-P)(f)$ is in $L^{2}(\nu)$. Define $h=f-G(\varphi)$. Then

$$
\begin{aligned}
(I-P)(h)(x) & =(I-P)(f-G(\varphi))(x) \\
& =\varphi(x)-G(\varphi)(x)+P G(\varphi)(x) \\
& =\varphi(x)-\sum_{n \in \mathbb{N}_{0}} P^{n}(\varphi)(x)+\sum_{n \in \mathbb{N}_{0}} P^{n+1}(\varphi)(x) \\
& =0 .
\end{aligned}
$$

Hence $h$ is harmonic and

$$
f=G(\varphi)+h .
$$

To see that this decomposition is unique, we suppose for contrary that for some function $f \in \mathcal{H}_{E}$

$$
f=G(\varphi)+h=G\left(\varphi^{\prime}\right)+h^{\prime} .
$$

Apply $(I-P)$ to both parts and obtain

$$
\varphi=(I-P)(G(\varphi)+h)=(I-P)\left(G\left(\varphi^{\prime}\right)+h^{\prime}\right)=\varphi^{\prime} .
$$

Therefore, $h=h^{\prime}$.

6.4. Structure of the energy space. We address now the question about the structure of the energy space $\mathcal{H}$. We first show that $\mathcal{H}$ contains the linear subspace spanned by characteristic functions of sets of finite measure. In the next statement we also give two formulas for computation of the norm of $\chi_{A}$ and the inner product of characteristic functions in terms of the measure $\rho$.

Lemma 6.18. Suppose $c(x)$ is locally integrable with respect to $\mu$. Then

$$
\mathcal{D}_{\text {fin }} \subset \mathcal{H} \text {. }
$$

Moreover, if $A \in \mathcal{B}_{\text {fin }}$, then

$$
\left\|\chi_{A}\right\|_{\mathcal{H}_{E}}^{2} \leq \int_{A} c(x) d \mu(x)
$$

and

$$
\left\|\chi_{A}\right\|_{\mathcal{H}_{E}}^{2}=\rho\left(A \times A^{c}\right)
$$

where $A^{c}:=V \backslash A$.

More generally,

$$
\left\langle\chi_{A}, \chi_{B}\right\rangle_{\mathcal{H}_{E}}=\rho((A \cap B) \times V)-\rho(A \times B)
$$

and

$$
\chi_{A} \perp \chi_{B} \Longleftrightarrow \rho((A \backslash B) \times B)=\rho\left((A \cap B) \times B^{c}\right) .
$$


Proof. We use (6.5) and compute for $A \in \mathcal{B}_{\text {fin }}$

$$
\begin{aligned}
\left\|\chi_{A}\right\|_{\mathcal{H}_{E}}^{2}= & \left.\frac{1}{2} \int_{V \times V}\left(\chi_{A}(x)-\chi_{A}(y)\right)^{2} d \rho(x, y) \quad \text { by (2.15) }\right) \\
= & \frac{1}{2} \int_{V \times V}\left(2 \chi_{A}(x)-2 \chi_{A}(x) \chi_{A}(y)\right) d \rho(x, y) \\
= & \int_{V} \int_{V} \chi_{A}(x) d \rho_{x}(y) d \mu(x) \\
& -\int_{V} \int_{V} \chi_{A}(x) \chi_{A}(y) d \rho_{x}(y) d \mu(x) \\
= & \int_{A} c(x) d \mu(x)-\int_{A} \rho_{x}(A) d \mu(x) \\
\leq & \int_{A} c(x) d \mu(x) \\
< &
\end{aligned}
$$

because, by the assumption, $c \in L_{\text {loc }}^{1}(\mu)$.

To see that (6.13) holds, we use the above relation and represent it in the convenient form

$$
\begin{aligned}
\left\|\chi_{A}\right\|_{\mathcal{H}_{E}}^{2} & =\int_{V} \int_{V} \chi_{A}(x) d \rho_{x}(y) d \mu(x)-\int_{V} \int_{V} \chi_{A}(x) \chi_{A}(y) d \rho_{x}(y) d \mu(x) \\
& =\int_{V \times V} \chi_{A \times V}(x, y) d \rho(x, y)-\int_{V \times V} \chi_{A \times A}(x, y) d \rho(x, y) \\
& =\int_{V \times V} \chi_{A \times A^{c}}(x, y) d \rho(x, y) \\
& =\rho\left(A \times A^{c}\right) .
\end{aligned}
$$

To finish the proof, we show that (6.14) holds. The computation is based on the definition, given in (6.5), and the property of symmetry for $\rho$ :

$$
\begin{aligned}
\left\langle\chi_{A}, \chi_{B}\right\rangle_{\mathcal{H}_{E}} & =\frac{1}{2} \int_{V \times V}\left(\chi_{A}(x)-\chi_{A}(y)\right)\left(\chi_{B}(x)-\chi_{B}(y)\right) d \rho(x, y) \\
& =\int_{V \times V}\left(\chi_{A}(x) \chi_{B}(x)-\chi_{A}(x) \chi_{B}(y)\right) d \rho(x, y) \\
& =\int_{V} \int_{V} \chi_{(A \cap B) \times V}(x, y) d \rho(x, y)-\int_{V} \int_{V} \chi_{A \times B}(x, y) d \rho(x, y) \\
& =\rho((A \cap B) \times V)-\rho(A \times B) .
\end{aligned}
$$

Lemma 6.19. Let $g$ be an element from $\mathcal{H}$ such that $\left\langle\chi_{A}, g\right\rangle_{\mathcal{H}_{E}}=0$ for every $A \in \mathcal{B}_{\text {fin }}$. Then $g$ is a harmonic function. 
Proof. By condition, the function $g(x)$ is orthogonal to every characteristic function $\chi_{A}, A \in \mathcal{B}_{\text {fin }}$. Then

$$
\begin{aligned}
0 & =\iint_{V \times V}\left(\chi_{A}(x)-\chi_{A}(y)\right)(g(x)-g(y)) d \rho(x, y) \\
& =\iint_{V \times V} \chi_{A}(x)(g(x)-g(y)) d \rho(x, y)-\iint_{V \times V} \chi_{A}(y)(g(x)-g(y)) d \rho(x, y) \\
& =\iint_{V \times V} \chi_{A}(x)(g(x)-g(y)) d \rho(x, y)-\iint_{V \times V} \chi_{A}(x)(g(y)-g(x)) d \rho(x, y) \\
& =2 \int_{V} \int_{V} \chi_{A}(x)(g(x)-g(y)) d \rho_{x}(y) d \mu(x) \\
& =2 \int_{V} \chi_{A}(x)(c(x) g(x)-R(g)(x)) d \mu(x) \\
& =2 \int_{A} c(x)(g(x)-P(g)(x)) d \mu(x) .
\end{aligned}
$$

Hence, $P(g)(x)=g(x)$ for $\mu$-a.e. $x \in V$, and Lemma is proved.

It follows from Lemma 6.19 that any harmonic function is orthogonal to the closure $\overline{\mathcal{D}}_{\text {fin }}$ of the space spanned by characteristic functions. Thus, we have the following decomposition.

We denote by $\mathcal{H a r m}_{E}$ the subspace in $\mathcal{H}$ such that $P(f)=f$, i.e.,

$$
\mathcal{H a r m}_{E}:=\{f \in \mathcal{F}(V, \mathcal{B}): P(f)=f\} \cap \mathcal{H} .
$$

Corollary 6.20. The finite energy space $\mathcal{H}$ admits the decomposition into the orthogonal sum

$$
\mathcal{H}=\overline{\mathcal{D}}_{\text {fin }} \oplus \mathcal{H} \text { arm }_{E}
$$

A function $h \in \mathcal{H}_{E}$ is harmonic if and only if

$$
\langle h, f-P(f)\rangle_{\mathcal{H}_{E}}=0 \quad \forall f \in \mathcal{D}_{\text {fin }} .
$$

Relation (6.15) is an extension of the Royden decomposition.

Proposition 6.21. Let $A \in \mathcal{B}_{\text {fin. }}$. Then $\left\|\chi_{A}\right\|_{\mathcal{H}_{E}}=0$ if and only if $\mu(A)=0$, i.e., $\chi_{A}=0$ a.e.

In the following statement, we collect several facts about the properties of characteristic functions considered as elements of the energy space.

Corollary 6.22. Let $\rho$ be a symmetric measure on $(V \times V, \mathcal{B} \times \mathcal{B})$, and $A, B \in \mathcal{B}_{\text {fin }}$. Under the assumption that $c \in L_{\mathrm{loc}}^{1}(\mu)$, the following statement hold:

(1) $\rho(A \times B)>0 \Longleftrightarrow \mu\left(\left\{x \in A: \rho_{x}(B)>0\right\}\right)>0$;

(2) $\chi_{A}=0$ in $\mathcal{H} \Longleftrightarrow\left\|\chi_{A}\right\|_{\mathcal{H}_{E}}=0 \Longleftrightarrow \rho_{x}(A)=c(x)$, p-a.e. $x \in A \Longleftrightarrow$ $\rho(A \times A)=\int_{A} c(x) d \mu(x)=\rho(A \times V)$;

(2a) in general, not assuming connectedness, $\left\|\chi_{A}\right\|_{\mathcal{H}_{E}}=0 \Longrightarrow P\left(\chi_{A} g\right)=$ $\chi_{A} P(g), \forall g \in \mathcal{H}_{E}$; 
(3)

$$
\chi_{A} \perp \chi_{B} \Longleftrightarrow \int_{A \cap B} c(x) d \mu(x)=\rho(A \times B)=\int_{A} \rho_{x}(B) d \mu(x) ;
$$

(4) if $A \subset B$ and $\mu(A)>0$, then

$$
\chi_{A} \perp \chi_{B} \Longleftrightarrow \rho\left(A \times B^{c}\right)=0 \Longleftrightarrow \rho_{x}\left(B^{c}\right)=0 \text { for a.e. } x \in A \text {. }
$$

(5) if $\chi_{A} \perp \chi_{B}$ and $A \cap B=\emptyset$, then $\rho(A \times B)=0$; in general, if $A \cap B=\emptyset$, then $\left\langle\chi_{A}, \chi_{B}\right\rangle_{\mathcal{H}_{E}} \leq 0$.

Proof. We begin with the obvious formula for the measure of a rectangle in $V \times V$ :

$$
\rho(A \times B)=\int_{A} \rho_{x}(B) d \mu(x), \quad A, B \in \mathcal{B}_{\text {fin }},
$$

where $\rho_{x}(B)=R\left(\chi_{B}\right)$. This proves $(1)$.

It was proved in Lemma 6.18 that

$$
\left\|\chi_{A}\right\|_{\mathcal{H}_{E}}^{2}=\int_{A}\left(c(x)-\rho_{x}(A)\right) d \mu(x)=\int_{A} \rho_{x}(V \backslash A) d \mu(x) .
$$

Because of (1), we see that

$$
\left\|\chi_{A}\right\|_{\mathcal{H}_{E}}=0 \Longleftrightarrow \rho(A \times A)=\rho(A \times V)
$$

This means that $(2)$ holds.

Since $\chi_{A}$ and $\chi_{B}$ are in $\mathcal{H}$, we can compute their inner product as in Lemma 6.18 .

$$
\left\langle\chi_{A}, \chi_{B}\right\rangle_{\mathcal{H}_{E}}=\iint_{V \times V}\left(\chi_{A}(x) \chi_{B}(x) d \rho(x, y)-\chi_{A}(x) \chi_{B}(y)\right) d \rho(x, y)
$$

Therefore, $\chi_{A} \perp \chi_{B}$ if and only if

$$
\iint_{V \times V}\left(\chi_{A}(x) \chi_{B}(x) d \rho(x, y)=\iint_{V \times V} \chi_{A}(x) \chi_{B}(y) d \rho(x, y) .\right.
$$

The latter is equivalent to

$$
\int_{V}\left(\chi_{A \cap B}(x)\left(\int_{V} d \rho_{x}(y)\right) d \mu(x)=\iint_{V \times V} \chi_{A}(x) \otimes \chi_{B}(y) d \rho(x, y)\right.
$$

or

$$
\int_{A \cap B} c(x) d \mu(x)=\int_{A} \rho_{x}(B) d \mu(x),
$$

that proves (3).

To show that (4) holds, we assume that $A \subset B$. Then it follows from (3) that

$$
\begin{aligned}
\chi_{A} \perp \chi_{B} & \Longleftrightarrow \int_{A} c(x) d \mu(x)=\int_{A} \rho_{x}(B) d \mu(x) ; \\
& \Longleftrightarrow \rho(A \times V)=\rho(A \times B) \\
& \Longleftrightarrow \rho\left(A \times B^{c}\right)=0 .
\end{aligned}
$$

Statement (5) follows from (3) and Lemma 6.18. 
Remark 6.23. As follows from the definition of the energy space, the zero element of $\mathcal{H}$ corresponds to any constant function. The proved properties of $\left\|\chi_{A}\right\|$ means that either the set $A$ or $A^{c}$ must have zero measure $\mu$.

Theorem 6.9 and Lemma 6.18 are used to describe the orthogonal complement of $\partial d(\mathcal{H})$ in $L^{2}(\rho)$.

Given a function $F(x, y) \in L^{2}(\rho)$, let $F^{\#}(x, y)$ denote the function $F(y, x)$.

Proposition 6.24. The orthogonal compliment $L^{2}(\rho) \ominus \partial(\mathcal{H})$ consists of all functions $F(x, y) \in L^{2}(\rho)$ such that $\widetilde{R}(F)(x)=\widetilde{R}\left(F^{\#}\right)(x)$ for $\mu$-a.e. $x \in V$ where the operator $\widetilde{R}$ is defined in (3.5).

Proof. Suppose that a function $F(x, y)$ belongs to $L^{2}(\rho) \ominus \partial(\mathcal{H})$. Then, for any $g(x) \in \mathcal{H}$, we have

$$
\begin{aligned}
\langle F(x, y), g(x)-g(y)\rangle_{L^{2}(\rho)}= & \int_{V \times V} F(x, y)(g(x)-g(y)) d \rho(x, y) \\
= & \int_{V \times V} F(x, y) g(x) d \rho(x, y) \\
& -\int_{V \times V} F(x, y) g(y) d \rho(x, y) \\
= & \int_{V \times V} F(x, y) g(x) d \rho(x, y) \\
& -\int_{V \times V} F^{\#}(x, y) g(x) d \rho(x, y) \\
= & \int_{V \times V} g(x)\left[F(x, y)-F^{\#}(x, y)\right] d \rho(x, y) \\
= & \int_{V} g(x)\left[\widetilde{R}(F)(x)-\widetilde{R}\left(F^{\#}\right)(x)\right] d \mu(x) \\
= & 0
\end{aligned}
$$

The above relation, in particular, holds for any characteristic function $g=\chi_{A}$, $A \in \mathcal{B}_{\text {fin. }}$. Hence, $\widetilde{R}(F)(x)-\widetilde{R}\left(F^{\#}\right)(x)=0$ a.e.

Clearly, the converse implication is also true.

Remark 6.25. In another applications of the kind of energy Hilbert space $\mathcal{H}_{E}$ we study is the last term in the Beurling-Dini formula, see App09, Theorem 3.6.5] and Example 2.16 (5); focus on the jump term. In our general setting, our $\mathcal{H}_{E}$ is paired with an $L^{2}$-space, and in the case of the standard Beurling-Dini formula, this $L^{2}$-space is $L^{2}\left(\mathbb{R}^{d}\right)$. For the literature, see e.g., [Kat68, AMR15, MR95.

\section{Spectral theOry for graph Laplacians in $L^{2}(\mu)$}

We will use here the notation introduced in the previous sections. In the next statement, we consider the graph Laplace operator $\Delta$ acting in the Hilbert space $L^{2}(\mu)$. To emphasize this fact, we will use also the notation $\Delta_{2}$. As usual, our 
basic objects are a measure space $(V, \mathcal{B}, \mu)$ and a symmetric measure $\rho$ such that $\rho_{x}(V)=c(x) \in(0, \infty)$ for $\mu$-a.e. $x \in V$.

Assumption $\boldsymbol{E}$ : We assume in this section that, for every set $A \in \mathcal{B}_{\text {fin }}$, the function

$$
x \mapsto \rho_{x}(A)=\int_{V} \chi_{A}(y) d \rho_{x}(y)
$$

belongs to $L^{1}(\mu) \cap L^{2}(\mu)$.

Recall that the subspace $\mathcal{D}_{\text {fin }}$ is spanned by characteristic functions $\chi_{A}$ with $\mu(A)<\infty$. Clearly, $\mathcal{D}_{\text {fin }}$ is dense in $L^{2}(\mu)$.

We use Assumption to justify the definition of the graph Laplace operator $\Delta$ as an unbounded linear operator acting in $L^{2}(\mu)$.

Lemma 7.1. Let

$$
\Delta(f)(x)=\int_{V}(f(x)-f(y)) d \rho_{x}(y)
$$

Then

$$
\mathcal{D}_{\text {fin }} \subset \operatorname{Dom}(\Delta) \cap L^{2}(\mu)
$$

and $\Delta$ is a densely defined operator.

Proof. It is obvious that $\mathcal{D}_{\text {fin }}$ is a dense subset in $L^{2}(\mu)$. We need to check only that $\Delta\left(\chi_{A}\right)$ is in $L^{2}(\mu)$ if $\mu(A)<\infty$. Since

$$
\Delta\left(\chi_{A}\right)(x)=c(x) \chi_{A}(x)-\rho_{x}(A),
$$

we conclude that $\Delta\left(\chi_{A}\right)$ is in $L^{2}(\mu)$ because of Assumption E.

Having the densely defined $\Delta$, we can associate the Hilbert adjoint operator $\Delta^{*}$, The domain of $\Delta^{*}$ is defined by the set of all elements $g$ of $\mathcal{H}$ for which the linear functional $f \mapsto\langle\Delta f, g\rangle_{L^{2}(\mu)}$ is continuous. Then there exists $g^{*} \in \mathcal{H}$ such that $\langle\Delta f, g\rangle_{L^{2}(\mu)}=\left\langle f, g^{*}\right\rangle_{L^{2}(\mu)}$. Set $\Delta^{*}(g)=g^{*}$. The operator $\Delta^{*}$ is uniquely defined.

In fact, we can determine $\Delta^{*}$ explicitly using the Identity

$$
\langle\Delta(f), g\rangle_{L^{2}(\mu)}=\left\langle f, \Delta^{*}(g)\right\rangle_{L^{2}(\mu)}
$$

and formula for $\Delta$ :

$$
\begin{aligned}
\langle\Delta(f), g\rangle_{L^{2}(\mu)} & =\int_{V} g(x)\left(\int_{V}(f(x)-f(y)) d \rho_{x}(y)\right) d \mu(x) \\
& =\iint_{V \times V}(g(x) f(x)-f(y) g(x)) d \rho(x, y) \\
& =\iint_{V \times V} g(x) f(x) d \rho(x, y)-\iint_{V \times V} f(x) g(y) d \rho(x, y) \\
& =\int_{V} f(x)\left(\int_{V}(g(x)-g(y)) d \rho_{x}(y)\right) d \mu(x) \\
& =\left\langle f, \Delta^{*}(g)\right\rangle_{L^{2}(\mu)} .
\end{aligned}
$$

Hence $\Delta^{*}(g)=\int_{V}(g(x)-g(y)) d \rho_{x}(y)$.

Therefore we have proved the following result: 
Proposition 7.2. The graph Laplace operator $\Delta$ considered in $L^{2}(\mu)$ is symmetric with dense domain $\mathcal{D}_{\text {fin }}$, i.e.,

$$
\langle g, \Delta(f)\rangle_{L^{2}(\mu)}=\langle\Delta(g), f\rangle_{L^{2}(\mu)} \text { on } \mathcal{D}_{\text {fin }} .
$$

We show below that, in fact, the graph Laplace operator $\Delta$ is self-adjoint.

Theorem 7.3. Let $\rho$ be a symmetric measure on $(V \times V, \mathcal{B} \times \mathcal{B})$ where $(V, \mathcal{B}, \mu)$ is a measure space. The graph Laplace operator $\Delta: L^{2}(\mu) \rightarrow L^{2}(\mu)$ is positive definite, i.e., it satisfies the following inequality:

$$
2 \int_{V} f^{2} c d \mu \geq\langle f, \Delta f\rangle_{L^{2}(\mu)} \geq 0, \quad \forall f \in \mathcal{D}_{\text {fin }}
$$

Proof. We first reformulate (7.2) in more convenient terms:

$$
\begin{aligned}
\langle f, \Delta f\rangle_{L^{2}(\mu)} & =\int_{V} f \Delta(f) d \mu \\
& =\int_{V} f(x)\left(\int_{V}(f(x)-f(y)) d \rho_{x}(y)\right) d \mu(x) \\
& =\int_{V} f^{2}(x) c(x) d \mu(x)-\int_{V} f(x)\left(\int_{V} f(y) d \rho_{x}(y)\right) d \mu(x)
\end{aligned}
$$

Hence $\langle f, \Delta f\rangle_{L^{2}(\mu)} \geq 0$ if and only if

$$
\int_{V} f^{2}(x) c(x) d \mu(x) \geq \int_{V} f(x)\left(\int_{V} f(y) d \rho_{x}(y)\right) d \mu(x)
$$

In order to prove (7.3), we apply the Schwarz inequality:

$$
\begin{aligned}
& \left|\int_{V} f(x)\left(\int_{V} f(y) d \rho_{x}(y)\right) d \mu(x)\right| \quad \text { (Schwarz inequality) } \\
& \leq \int_{V}|f(x)|\left(\int_{V} f^{2}(y) d \rho_{x}(y)\right)^{1 / 2}\left(\int_{V} d \rho_{x}(y)\right)^{1 / 2} d \mu(x) \\
& =\int_{V}|f(x)| \sqrt{c(x)}\left(\int_{V} f^{2}(y) d \rho_{x}(y)\right)^{1 / 2} d \mu(x) \quad \text { (Schwarz inequality) } \\
& \leq\left(\int_{V} f^{2} c d \mu\right)^{1 / 2}\left(\int_{V} \rho_{x}\left(f^{2}\right) d \mu\right)^{1 / 2} \\
& =\left(\int_{V} f^{2} c d \mu\right)^{1 / 2}\left(\int_{V} f^{2} c d \mu\right)^{1 / 2} \\
& =\int_{V} f^{2} c d \mu .
\end{aligned}
$$

We used here the fact that

$$
\int_{V} g c d \mu=\int_{V} R(g) d \mu=\int_{V} \rho_{x}(g) d \mu .
$$

This proves (7.3). Therefore, $\Delta$ is positive definite. 
To see that the other inequality in (7.2) holds, we consider (7.3) and write it as

$$
\langle f, \Delta f\rangle_{L^{2}(\mu)} \leq \int_{V} f^{2}(x) c(x) d \mu(x)+\left|\int_{V} f(x)\left(\int_{V} f(y) d \rho_{x}(y)\right) d \mu(x)\right|
$$

The result then follows from (7.3).

Corollary 7.4. The operator $\Delta$ acting in $L^{2}(\mu)$ is bounded if and only if $c \in L^{\infty}(\mu)$. Proof. The result follows immediately from the inequality

$$
2 \int_{V} f^{2} c d \mu \geq\langle f, \Delta f\rangle_{L^{2}(\mu)}
$$

Theorem 7.5. Let $f$ be an element of the energy space such that $f$ and $\Delta(f)$ are elements of $L^{2}(\mu)$. Then

$$
\|f\|_{\mathcal{H}}^{2}=\int_{V} f \Delta(f) d \mu .
$$

Proof. We first observe that the condition of the theorem holds for any function $f \in \mathcal{D}_{\text {fin. }}$. We compute the norm of $f$ in $\mathcal{H}$ by using the symmetric property of the measure $\rho$. In other words, the equality

$$
\int_{V} f(y) d \rho(x, y)=\int_{V} f(x) d \rho(x, y)
$$

holds for any function $f$. Therefore, we have

$$
\begin{aligned}
\|f\|_{\mathcal{H}}^{2} & =\frac{1}{2} \iint_{V \times V}(f(x)-f(y))^{2} d \rho(x, y) \\
& =\iint_{V \times V}\left[f^{2}(x)-f(x) f(y)\right] d \rho(x, y) \\
& =\int_{V}\left(\int_{V}\left[f(x)^{2}-f(x) f(y)\right] d \rho_{x}(y)\right) d \mu(x) \\
& =\int_{V}\left[f(x)^{2} c(x)-f(x) R(f)(x)\right] d \mu(x) \\
& =\int_{V} f(x)[c(x) f(x)-R(f)(x)] d \mu(x) \\
& =\int_{V} f(x) \Delta(f)(x) d \mu(x) .
\end{aligned}
$$

Hence, If $f$ and $\Delta(f)$ are $\in L^{2}(\mu)$, then we obtain that

$$
\|f\|_{\mathcal{H}}^{2}=\langle f, \Delta f\rangle_{L^{2}(\mu)}
$$

Remark 7.6. In (7.4), we can use the equality

$$
\int_{V} f \Delta(f) d \mu=\langle f, \Delta(f)\rangle_{L^{2}(\mu)}
$$


only for those functions $f$ from $\mathcal{H}$ which are also in $L^{2}(\mu)$. If $f$ is not in $L^{2}(\mu)$, the integral in (7.4) is still well defined. It is worth noting that, in general, Theorem 7.5 does not hold for arbitrary functions $f$ from $\mathcal{H}$. In the case of discrete networks, it was shown in [JP11, JP13] that a certain discrete Gauss-Green formula contains an additional term, the so called boundary term.

We are ready to prove our main result of this section.

Theorem 7.7. The graph Laplace operator $\Delta$ is self-adjoint in the Hilbert space $L^{2}(\mu)$.

Proof. We showed that $\Delta$ is a symmetric operator. In order to proof that it is self-adjoint, it suffices to show that the deficiency index of $\Delta$ is zero.

Lemma 7.8. $\Delta^{*} u=-u \Longrightarrow u=0$.

Proof of the lemma. Since $\Delta$ is symmetric and $\Delta^{*} u=\Delta u=c(u-P u)$, we show that the equality

$$
c(u-P u)=-u
$$

holds only when $=0$. Relation (7.5) is equivalent to

$$
P u=\left(1+\frac{1}{c}\right) u \text {. }
$$

We use the fact proved in Theorem 7.3 that $\Delta$ is positive definite:

$$
\begin{aligned}
\langle u, \Delta u\rangle_{L^{2}(\mu)} & =\langle u, c(u-P u)\rangle_{L^{2}(\mu)} \geq 0 \\
\mathbb{1} & \\
\int_{V} c u^{2} d \mu & \geq \int_{V} c u P(u) d \mu \\
\mathbb{1} & \\
\int_{V} c u^{2} d \mu & \geq \int_{V} c u\left(1+c^{-1}\right) u d \mu \\
\mathbb{1} & \\
\int_{V} c u^{2} d \mu & \geq \int_{V} c u^{2} d \mu+\int_{V} u^{2} d \mu
\end{aligned}
$$

Hence $u=0$ in $L^{2}(\mu)$, and this completes the proof of the theorem.

Corollary 7.9. Let $f, g \in \mathcal{H}_{E}$ be two functions such that $f, g$ and $\Delta f, \Delta g$ belong to $L^{2}(\mu)$. Then

$$
\langle f, g\rangle_{\mathcal{H}_{E}}=\langle f, \Delta g\rangle_{L^{2}(\mu)}
$$

Proof. Relation (7.6) immediately follows from two facts: formula (6.4) of Theorem 7.5. applied to $\|f+g\|_{\mathcal{H}_{E}}$, and the self-adjointness of the operator $\Delta$ in $L^{2}(\mu)$, Theorem 7.7 .

For more details regarding the potential theory and finite energy space (Dirichlet space), the reader may consult the following items [Kan06, Kan14, KR07] and the papers cited there. 


\section{Spectral theory of the graph Laplacian in the energy space}

In this section, we consider the graph Laplace operator $\Delta$ acting in the energy space $\mathcal{H}=\mathcal{H}_{E}$. We will also discuss the properties of this operator $\Delta$.

Our approach is based on the notion of symmetric pairs of operators. We briefly describe this approach. For more details regarding the theory of unbounded operators, readers may consult the following items [DS88, JT17b] and the papers cited there.

Let $\mathcal{H}_{1}$ and $\mathcal{H}_{2}$ be Hilbert spaces, and let $\mathcal{D}_{1} \subset \mathcal{H}_{1}$ and $\mathcal{D}_{2} \subset \mathcal{H}_{2}$ be dense subspaces. Suppose that two linear operators

$$
J: \mathcal{D}_{1} \rightarrow \mathcal{H}_{2}, \quad K: \mathcal{D}_{2} \rightarrow \mathcal{H}_{1}
$$

are defined on these dense subspaces. The pair $(J, K)$ is called a symmetric pair if

$$
\langle J \varphi, \psi\rangle_{\mathcal{H}_{2}}=\langle\varphi, K \psi\rangle_{\mathcal{H}_{1}}, \quad \varphi \in \mathcal{D}_{1}, \psi \in \mathcal{D}_{2} .
$$

The following statement is a well known result in the theory of unbounded operators.

Lemma 8.1. (1) Suppose ( $J, K)$ be a symmetric pair satisfying (8.1) and (8.2). Then the operators $J$ and $K$ are closable and $J \subset K^{*}, K \subset J^{*}$. Without loss of generality, one can assume that $J=\bar{J}, K=\bar{K}$.

(2) $J^{*} J$ is a self-adjoint densely defined operator in $\mathcal{H}_{1}$, and $K^{*} K$ is a self-adjoint densely defined operator in $\mathcal{H}_{2}$.

Now we apply the above statement to the case of Hilbert spaces $L^{2}(\mu)$ and $\mathcal{H}_{E}$. To distinguish the graph Laplace operators acting in $L^{2}(\mu)$ and $\mathcal{H}_{E}$, we will use the notation $\Delta_{2}$ and $\Delta_{\mathcal{H}}$, respectively.

As was proved in Theorems 7.3 and 7.7 , the operator $\Delta_{2}$ is positive definite and essentially self-adjoint; therefore, by the spectral theorem, there exists a projectionvalued measure $Q(d t)$ such that

$$
\Delta_{2}=\int_{0}^{\infty} t d Q(t)
$$

or, for any $\varphi \in L^{2}(\mu)$,

$$
\left\langle\varphi, \Delta_{2} \varphi\right\rangle_{L^{2}(\mu)}=\int_{0}^{\infty} t\|Q(d t) \varphi\|_{L^{2}(\mu)}^{2}
$$

(we used here the fact that $Q(d t)$ is a projection).

Lemma 8.2. In the above notation, let

$$
H_{n, m}=Q\left(\left[n^{-1}, m\right]\right) L^{2}(\mu), \quad n, m \in \mathbb{N} .
$$

Then

$$
\mathcal{D}_{Q}:=\bigcup_{n, m} H_{n, m}
$$

is a dense subspace in $L^{2}(\mu)$ which is also invariant with respect to $\Delta_{2}$ and $\Delta_{2}^{-1}$.

Moreover, $\mathcal{D}_{Q}$ can be viewed also as a subspace of $\mathcal{H}_{E}$. 
Proof. The density of $\mathcal{D}_{Q}$ follows directly from the spectral theorem since the double-indexed sequence of projections $\left\{Q\left(\left[n^{-1}, m\right]\right)\right\}$ strongly converges to the identity operator $I$ in $L^{2}(\mu)$ as $n, m \rightarrow \infty$. The invariance of $\mathcal{D}_{Q}$ with respect to $\Delta_{2}$ and $\Delta_{2}^{-1}$ is deduced from the boundness of $\Delta_{2}$ and $\Delta_{2}^{-1}$ on every set $H_{n, m}$. We see that

$$
n^{-1}\|\varphi\|_{L^{2}(\mu)} \leq\left\|\Delta_{2} \varphi\right\|_{L^{2}(\mu)} \leq m\|\varphi\|_{L^{2}(\mu)}, \quad \varphi \in H_{n, m} .
$$

Similarly, for $\varphi \in H_{n, m}$, we have

$$
m^{-1}\|\varphi\|_{L^{2}(\mu)} \leq\left\|\Delta_{2}^{-1} \varphi\right\|_{L^{2}(\mu)} \leq n\|\varphi\|_{L^{2}(\mu)}
$$

because

$$
\Delta_{2}^{-1}=\int_{0}^{\infty} t^{-1} d Q(t)
$$

Hence, if $\varphi$ is in $\mathcal{D}_{Q}$, then $\Delta_{2} \varphi \in \mathcal{D}_{Q}$ and $\Delta_{2}^{-1} \varphi \in \mathcal{D}_{Q}$.

The proof of the second assertion of the lemma follows from relation (7.4). We have

$$
\|f\|_{\mathcal{H}_{E}}^{2}=\left\langle f, \Delta_{2} f\right\rangle_{L^{2}(\mu)}
$$

and this holds for any function $f \in \mathcal{D}_{Q} \subset L^{2}(\mu)$ (note that then $\Delta_{2} f$ is in $\mathcal{D}_{Q}$ ). It follows from (8.4) that, for $f \in H_{n, m}$,

$$
\|f\|_{\mathcal{H}_{E}}^{2} \leq m\|f\|_{L^{2}(\mu)}
$$

Lemma 8.3. Let Harm be the set of harmonic functions in $\mathcal{H}_{E}$. Then the space

$$
\mathcal{C}:=\mathcal{D}_{Q}+\text { Harm }
$$

is dense in $\mathcal{H}_{E}$.

Proof. This result follows from the inclusion $\mathcal{D}_{\text {fin }} \subset \mathcal{D}_{Q}$ and the decomposition (6.15).

We now define two operators, $J$ and $K$, that constitute a symmetric pair. Based on Lemmas 8.3 and 8.2 , we can define the densely defined operator $J$ :

$$
L^{2}(\mu) \supset \mathcal{D}_{Q} \ni \varphi \stackrel{J}{\longrightarrow} \varphi \in \mathcal{H}_{E} .
$$

To define its counterpart, the operator $K$, we use Lemma 8.3 and put

$$
K h=0, h \in H a r m, \quad K \psi=\Delta_{2} \psi, \psi \in D_{Q},
$$

where $\operatorname{Harm}$ is the set of harmonic functions in $\mathcal{H}_{E}$. Then $K$ is a densely defined operator on the subspace $\mathcal{C}$ of $\mathcal{H}_{E}$ such that $K(\mathcal{C}) \subset L^{2}(\mu)$.

Lemma 8.4. The operators $J$ and $K$, defined by (8.5) and (8.6), form a symmetric pair, i.e.,

$$
\langle J \varphi, \psi\rangle_{\mathcal{H}_{E}}=\langle\varphi, K \psi\rangle_{L^{2}(\mu)}, \quad \varphi \in \mathcal{D}_{Q}, \psi \in \mathcal{C} .
$$


Proof. We first observe that, by Lemma 8.3. every function $\psi \in \mathcal{D}_{Q}$ can be represented as $\psi=\Delta_{2}^{-1} \xi$. Then we use Corollary 7.9 in the proof. By definition of $K$, we obtain that, for $\varphi, \psi \in \mathcal{D}_{Q}$,

$$
\begin{aligned}
\langle J \varphi, \psi\rangle_{\mathcal{H}_{E}} & =\left\langle J \varphi, \Delta_{2}^{-1} \xi\right\rangle_{\mathcal{H}_{E}} \\
& =\left\langle\varphi, \Delta_{2}\left(\Delta_{2}^{-1} \xi\right)\right\rangle_{L^{2}(\mu)} \\
& =\langle\varphi, K \psi\rangle_{L^{2}(\mu)}
\end{aligned}
$$

If $\psi$ is in Harm, then the left- and right-hand sides in (8.7) are simultaneously equal to zero.

It follows from Lemma 8.4 that:

(1) $J^{*}=K$ and $K^{*}=J$,

(2) the operators $J^{*} J$ and $K^{*} K$ are self-adjoint in $L^{2}(\mu)$ and $\mathcal{H}_{E}$, respectively.

We combine the results of the lemmas proved in this section in the following theorem.

Let $\Delta$ be a linear operator acting on Borel functions $f \in \mathcal{F}(X, \mathcal{B})$ by

$$
\Delta(f)(x)=\int_{V}(f(x)-f(y)) d \rho_{x}(y)
$$

where $\rho=\int_{V} \rho_{x} d \mu(x)$ is a symmetric measure.

Theorem 8.5. The Laplace operator $\Delta$ admits its realizations in the Hilbert spaces $L^{2}(\mu)$ and $\mathcal{H}_{E}$ such that:

(i) $\Delta_{2}=J^{*} J$ is a positive definite essentially self-adjoint operator;

(ii) $\Delta_{\mathcal{H}}$ is a positive definite and symmetric operator which is not self-adjoint, in general; a self-adjoint extension of $\Delta_{\mathcal{H}}$ is given by the opearor $J J^{*}=K^{*} K$.

Remark 8.6. (1) The operator $\Delta_{\mathcal{H}}$ has a self-adjoint extension $J J^{*}$ but its deficiency indices might be non-zero. Corresponding examples can be found in the discrete theory of Laplace operators (see [JP16]).

(2) We recall that $\mathcal{D}_{\text {fin }}$ is a natural dense subset in the Hilbert space $L^{2}(\mu)$. Moreover, functions from $\mathcal{D}_{\text {fin }}$ belong to the energy space $\mathcal{H}_{E}$. Then we could define the operator $J$ by putting

$$
\chi_{A} \stackrel{J}{\longrightarrow} \chi_{A}: L_{2}(\mu) \rightarrow \mathcal{H}_{E} .
$$

But for the definition of the operator $K$ we do need the dense subset $\mathcal{C}$ in the energy space $\mathcal{H}_{E}$. Then the conclusion of Theorem 8.5 can then be obtained from the pair $J, K$.

(3) The definition of the operator $\Delta_{E}$ is based on the construction of a symmetric pair of operators. A similar technique can be used to define an analogue of the Markov operator $P$ acting in the space $\mathcal{H}_{E}$. We briefly outline this approach.

Let the operators $\partial: \mathcal{H}_{E} \rightarrow$ Diss and $S:$ Diss $\rightarrow$ Diss be defined by (6.9) and (5.6), respectively.

Claim. The operator $P: \mathcal{H}_{E} \rightarrow \mathcal{H}_{E}$ is defined by the formula

$$
P=\partial^{*} S \partial \text {. }
$$


In other words, the following diagram is commutative.

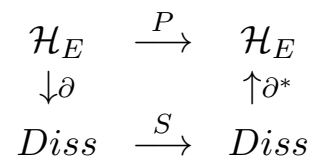

We take now $J$ as in (8.8) and define $K: \mathcal{H}_{E} \rightarrow L^{2}(\nu)$ by the formula (see Corollary 6.17)

$$
K: f=G(\varphi)+h \mapsto \varphi .
$$

Then one can check that $J$ and $K$ form a symmetric pair, i.e., $K=J^{*}$. Using this pair we can define an operator $P: \mathcal{H}_{E} \rightarrow \mathcal{H}_{E}$, an analogue of the Markov operator on $L^{2}(\nu)$.

Acknowledgments. The authors are pleased to thank colleagues and collaborators, especially members of the seminars in Mathematical Physics and Operator Theory at the University of Iowa, where versions of this work have been presented. We acknowledge very helpful conversations with among others Professors Paul Muhly, Wayne Polyzou; and conversations at distance with Professors Daniel Alpay, and his colleagues at both Ben Gurion University, and Chapman University. The second named author presented an early version of our paper at a 2017-Chapman University conference, Signal Processing and Linear Systems: New Problems and Directions.

\section{REFERENCES}

[Aar97] Jon Aaronson. An introduction to infinite ergodic theory, volume 50 of Mathematical Surveys and Monographs. American Mathematical Society, Providence, RI, 1997.

[AFMP94] Gregory T. Adams, John Froelich, Paul J. McGuire, and Vern I. Paulsen. Analytic reproducing kernels and factorization. Indiana Univ. Math. J., 43(3):839-856, 1994.

[AJ12] Daniel Alpay and Palle E. T. Jorgensen. Stochastic processes induced by singular operators. Numer. Funct. Anal. Optim., 33(7-9):708-735, 2012.

[AJ14] Daniel Alpay and Palle Jorgensen. Reproducing kernel Hilbert spaces generated by the binomial coefficients. Illinois J. Math., 58(2):471-495, 2014.

[AJ15] Daniel Alpay and Palle Jorgensen. Spectral theory for Gaussian processes: reproducing kernels, boundaries, and $L^{2}$-wavelet generators with fractional scales. Numer. Funct. Anal. Optim., 36(10):1239-1285, 2015.

[AJK15] Daniel Alpay, Palle E. T. Jorgensen, and David P. Kimsey. Moment problems in an infinite number of variables. Infin. Dimens. Anal. Quantum Probab. Relat. Top., 18(4):1550024, 14, 2015.

[AJL11] Daniel Alpay, Palle Jorgensen, and David Levanony. A class of Gaussian processes with fractional spectral measures. J. Funct. Anal., 261(2):507-541, 2011.

[AJL17] Daniel Alpay, Palle Jorgensen, and David Levanony. On the equivalence of probability spaces. J. Theoret. Probab., 30(3):813-841, 2017.

[AJLM15] Daniel Alpay, Palle Jorgensen, Izchak Lewkowicz, and Itzik Martziano. Infinite product representations for kernels and iterations of functions. In Recent advances in inverse scattering, Schur analysis and stochastic processes, volume 244 of Oper. Theory Adv. Appl., pages 67-87. Birkhäuser/Springer, Cham, 2015.

[AJSV13] Daniel Alpay, Palle Jorgensen, Ron Seager, and Dan Volok. On discrete analytic functions: products, rational functions and reproducing kernels. J. Appl. Math. Comput., 41(1-2):393-426, 2013. 
[AJV14] Daniel Alpay, Palle Jorgensen, and Dan Volok. Relative reproducing kernel Hilbert spaces. Proc. Amer. Math. Soc., 142(11):3889-3895, 2014.

[AMR15] Sergio Albeverio, Zhi-Ming Ma, and Michael Röckner. Quasi regular Dirichlet forms and the stochastic quantization problem. In Festschrift Masatoshi Fukushima, volume 17 of Interdiscip. Math. Sci., pages 27-58. World Sci. Publ., Hackensack, NJ, 2015.

[App09] David Applebaum. Lévy processes and stochastic calculus, volume 116 of Cambridge Studies in Advanced Mathematics. Cambridge University Press, Cambridge, second edition, 2009.

[APSS17] Marco Avella-Medina, Francesca Parise, Michael T. Schaub, and Santiago Segarra. Centrality measures for graphons. CoRR, abs/1707.09350, 2017.

[Aro50] N. Aronszajn. Theory of reproducing kernels. Trans. Amer. Math. Soc., 68:337-404, 1950.

[Atk75] Kendall Atkinson. Convergence rates for approximate eigenvalues of compact integral operators. SIAM J. Numer. Anal., 12:213-222, 1975.

$\left[\mathrm{BCL}^{+} 08\right]$ C. Borgs, J. T. Chayes, L. Lovász, V. T. Sós, and K. Vesztergombi. Convergent sequences of dense graphs. I. Subgraph frequencies, metric properties and testing. $A d v$. Math., 219(6):1801-1851, 2008.

$\left[\mathrm{BCL}^{+} 12\right]$ C. Borgs, J. T. Chayes, L. Lovász, V. T. Sós, and K. Vesztergombi. Convergent sequences of dense graphs II. Multiway cuts and statistical physics. Ann. of Math. (2), 176(1):151-219, 2012.

[BJ15a] S. Bezuglyi and P. E. T. Jorgensen. Monopoles, dipoles, and harmonic functions on Bratteli diagrams. ArXiv e-prints, August 2015.

[BJ15b] S. Bezuglyi and Palle E. T. Jorgensen. Representations of Cuntz-Krieger relations, dynamics on Bratteli diagrams, and path-space measures. In Trends in harmonic analysis and its applications, volume 650 of Contemp. Math., pages 57-88. Amer. Math. Soc., Providence, RI, 2015.

[BJ17] S. Bezuglyi and P. E. T. Jorgensen. Infinite-dimensional transfer operators, endomorphisms, and measurable partitions. ArXiv e-prints, February 2017.

$\left[\mathrm{BLP}^{+} 10\right]$ Lawrence W. Baggett, Nadia S. Larsen, Judith A. Packer, Iain Raeburn, and Arlan Ramsay. Direct limits, multiresolution analyses, and wavelets. J. Funct. Anal., 258(8):2714-2738, 2010.

[BO17] Alexei Borodin and Grigori Olshanski. The ASEP and determinantal point processes. Comm. Math. Phys., 353(2):853-903, 2017.

[BQ15] Alexander I. Bufetov and Yanqi Qiu. Equivalence of Palm measures for determinantal point processes associated with Hilbert spaces of holomorphic functions. C. R. Math. Acad. Sci. Paris, 353(6):551-555, 2015.

$\left[\mathrm{CGG}^{+}{ }^{16}\right.$ ] Chao-Yang Chen, Weihua Gui, Zhi-Hong Guan, Shaowu Zhou, and Cailun Huang. Optimal tracking performance of MIMO discrete-time systems with network parameters. Discrete Dyn. Nat. Soc., pages Art. ID 6826130, 7, 2016.

[Cho14] Ilwoo Cho. Algebras, graphs and their applications. CRC Press, Boca Raton, FL, 2014. Edited by Palle E. T. Jorgensen.

[CWK17] Yi-Chun Chen, Tim A. Wheeler, and Mykel J. Kochenderfer. Learning discrete Bayesian networks from continuous data. J. Artificial Intelligence Res., 59:103-132, 2017.

[CZ07] Felipe Cucker and Ding-Xuan Zhou. Learning theory: an approximation theory viewpoint, volume 24 of Cambridge Monographs on Applied and Computational Mathematics. Cambridge University Press, Cambridge, 2007. With a foreword by Stephen Smale.

[DJ06] Dorin Ervin Dutkay and Palle E. T. Jorgensen. Iterated function systems, Ruelle operators, and invariant projective measures. Math. Comp., 75(256):1931-1970, 2006. 
[DJ11] Dorin Ervin Dutkay and Palle E. T. Jorgensen. Spectral duality for unbounded operators. J. Operator Theory, 65(2):325-353, 2011.

[DS88] Nelson Dunford and Jacob T. Schwartz. Linear operators. Part II. Wiley Classics Library. John Wiley \& Sons, Inc., New York, 1988. Spectral theory. Selfadjoint operators in Hilbert space, With the assistance of William G. Bade and Robert G. Bartle, Reprint of the 1963 original, A Wiley-Interscience Publication.

[EFHN15] Tanja Eisner, Bálint Farkas, Markus Haase, and Rainer Nagel. Operator theoretic aspects of ergodic theory, volume 272 of Graduate Texts in Mathematics. Springer, Cham, 2015.

[Gei17] Bernhard C. Geiger. A sufficient condition for a unique invariant distribution of a higher-order Markov chain. Statist. Probab. Lett., 130:49-56, 2017.

[Gho15] Subhroshekhar Ghosh. Determinantal processes and completeness of random exponentials: the critical case. Probab. Theory Related Fields, 163(3-4):643-665, 2015.

[GQ15] Stéphane Gaubert and Zheng Qu. Dobrushin's ergodicity coefficient for Markov operators on cones. Integral Equations Operator Theory, 81(1):127-150, 2015.

[HKPV09] J. Ben Hough, Manjunath Krishnapur, Yuval Peres, and Bálint Virág. Zeros of Gaussian analytic functions and determinantal point processes, volume 51 of University Lecture Series. American Mathematical Society, Providence, RI, 2009.

[Ho17] Kwok-Pun Ho. Modular estimates of fractional integral operators and $k$-plane transforms. Integral Transforms Spec. Funct., 28(11):801-812, 2017.

[Jan13] Svante Janson. Graphons, cut norm and distance, couplings and rearrangements, volume 4 of New York Journal of Mathematics. NYJM Monographs. State University of New York, University at Albany, Albany, NY, 2013.

[JKL02] S. Jackson, A. S. Kechris, and A. Louveau. Countable Borel equivalence relations. $J$. Math. Log., 2(1):1-80, 2002.

[Jor01] Palle E. T. Jorgensen. Ruelle operators: functions which are harmonic with respect to a transfer operator. Mem. Amer. Math. Soc., 152(720):viii+60, 2001.

[Jor12] Palle E. T. Jorgensen. Unbounded graph-Laplacians in energy space, and their extensions. J. Appl. Math. Comput., 39(1-2):155-187, 2012.

[JP11] Palle E. T. Jorgensen and Erin P. J. Pearse. Resistance boundaries of infinite networks. In Random walks, boundaries and spectra, volume 64 of Progr. Probab., pages 111-142. Birkhäuser/Springer Basel AG, Basel, 2011.

[JP12] P. E. T. Jorgensen and A. M. Paolucci. Markov measures and extended zeta functions. J. Appl. Math. Comput., 38(1-2):305-323, 2012.

[JP13] Palle E. T. Jorgensen and Erin P. J. Pearse. A discrete Gauss-Green identity for unbounded Laplace operators, and the transience of random walks. Israel J. Math., 196(1):113-160, 2013.

[JP14] Palle E. T. Jorgensen and Erin P. J. Pearse. Spectral comparisons between networks with different conductance functions. J. Operator Theory, 72(1):71-86, 2014.

[JP16] Palle E. T. Jorgensen and Erin P. J. Pearse. Symmetric pairs and self-adjoint extensions of operators, with applications to energy networks. Complex Anal. Oper. Theory, 10(7):1535-1550, 2016.

[JP17] Palle E. T. Jorgensen and Erin P. J. Pearse. Symmetric pairs of unbounded operators in Hilbert space, and their applications in mathematical physics. Math. Phys. Anal. Geom., 20(2):Art. 14, 24, 2017.

[JPT16] Palle Jorgensen, Steen Pedersen, and Feng Tian. Extensions of positive definite functions, volume 2160 of Lecture Notes in Mathematics. Springer, [Cham], 2016. Applications and their harmonic analysis.

[JS13] Palle E. T. Jorgensen and Myung-Sin Song. Compactification of infinite graphs and sampling. Sampl. Theory Signal Image Process., 12(2-3):139-158, 2013.

[JT15a] Palle Jorgensen and Feng Tian. Discrete reproducing kernel Hilbert spaces: sampling and distribution of Dirac-masses. J. Mach. Learn. Res., 16:3079-3114, 2015. 
[JT15b] Palle Jorgensen and Feng Tian. Frames and factorization of graph Laplacians. Opuscula Math., 35(3):293-332, 2015.

[JT15c] Palle Jorgensen and Feng Tian. Infinite networks and variation of conductance functions in discrete Laplacians. J. Math. Phys., 56(4):043506, 27, 2015.

[JT17a] P. Jorgensen and F. Tian. Transfer operators, induced probability spaces, and random walk models. Markov Process. Related Fields, 23(2):187-210, 2017.

[JT17b] Palle Jorgensen and Feng Tian. Non-commutative analysis. World Scientific Publishing Co. Pte. Ltd., Hackensack, NJ, 2017. With a foreword by Wayne Polyzou.

[Kal83] Olav Kallenberg. Random measures. Akademie-Verlag, Berlin; Academic Press, Inc. [Harcourt Brace Jovanovich, Publishers], London, third edition, 1983.

[Kan06] Hiroshi Kaneko. Sobolev space and Dirichlet space associated with symmetric Markov process on a local field. Potential Anal., 24(1):87-103, 2006.

[Kan08] Vladimir Kanovei. Borel equivalence relations, volume 44 of University Lecture Series. American Mathematical Society, Providence, RI, 2008. Structure and classification.

[Kan14] Hiroshi Kaneko. A Dirichlet space on ends of tree and Dirichlet forms with a nodewise orthogonal property. Potential Anal., 41(1):245-268, 2014.

[Kat68] Yitzhak Katznelson. An introduction to harmonic analysis. John Wiley \& Sons, Inc., New York-London-Sydney, 1968.

[Kec10] Alexander S. Kechris. Global aspects of ergodic group actions, volume 160 of Mathematical Surveys and Monographs. American Mathematical Society, Providence, RI, 2010.

[KM15] RafałKapica and Janusz Morawiec. Inhomogeneous poly-scale refinement type equations and Markov operators with perturbations. J. Fixed Point Theory Appl., 17(3):507-520, 2015.

[Kol50] A. N. Kolmogorov. Foundations of the Theory of Probability. Chelsea Publishing Company, New York, N. Y., 1950.

[KR07] Hiroshi Kawabi and Michael Röckner. Essential self-adjointness of Dirichlet operators on a path space with Gibbs measures via an SPDE approach. J. Funct. Anal., 242(2):486-518, 2007.

[Lov12] László Lovász. Large networks and graph limits, volume 60 of American Mathematical Society Colloquium Publications. American Mathematical Society, Providence, RI, 2012.

[LP16] Russell Lyons and Yuval Peres. Probability on trees and networks, volume 42 of Cambridge Series in Statistical and Probabilistic Mathematics. Cambridge University Press, New York, 2016.

[Luk16] Taras Lukashiv. One form of Lyapunov operator for stochastic dynamic system with Markov parameters. J. Math., pages Art. ID 1694935, 5, 2016.

[Lyo03] Russell Lyons. Determinantal probability measures. Publ. Math. Inst. Hautes Études Sci., (98):167-212, 2003.

[MR92] Zhi Ming Ma and Michael Röckner. Introduction to the theory of (nonsymmetric) Dirichlet forms. Universitext. Springer-Verlag, Berlin, 1992.

[MR95] Zhi Ming Ma and Michael Röckner. Markov processes associated with positivity preserving coercive forms. Canad. J. Math., 47(4):817-840, 1995.

[PR16] Vern I. Paulsen and Mrinal Raghupathi. An introduction to the theory of reproducing kernel Hilbert spaces, volume 152 of Cambridge Studies in Advanced Mathematics. Cambridge University Press, Cambridge, 2016.

[Rev84] D. Revuz. Markov chains, volume 11 of North-Holland Mathematical Library. NorthHolland Publishing Co., Amsterdam, second edition, 1984.

[Roh49] V. A. Rohlin. On the fundamental ideas of measure theory. Mat. Sbornik N.S., 25(67):107-150, 1949.

[Rud87] Walter Rudin. Real and complex analysis. McGraw-Hill Book Co., New York, third edition, 1987. 
[Sim12] David Simmons. Conditional measures and conditional expectation; Rohlin's disintegration theorem. Discrete Contin. Dyn. Syst., 32(7):2565-2582, 2012.

[SS17] Itaru Sasaki and Akito Suzuki. Essential spectrum of the discrete Laplacian on a perturbed periodic graph. J. Math. Anal. Appl., 446(2):1863-1881, 2017.

[Ter17] Dalia Terhesiu. Non trivial limit distributions for transient renewal chains. Statist. Probab. Lett., 129:189-195, 2017.

[Ver00] A. M. Vershik. Dynamic theory of growth in groups: entropy, boundaries, examples. Uspekhi Mat. Nauk, 55(4(334)):59-128, 2000.

[Ver05] A. M. Vershik. Polymorphisms, Markov processes, and quasi-similarity. Discrete Contin. Dyn. Syst., 13(5):1305-1324, 2005.

[Vil09] Cédric Villani. Optimal transport, volume 338 of Grundlehren der Mathematischen Wissenschaften [Fundamental Principles of Mathematical Sciences]. Springer-Verlag, Berlin, 2009. Old and new.

[Yos95] Kōsaku Yosida. Functional analysis. Classics in Mathematics. Springer-Verlag, Berlin, 1995. Reprint of the sixth (1980) edition.

Department of Mathematics, University of Iowa, Iowa City, 52242 IA, USA

E-mail address: sergii-bezuglyi@uiowa.edu

E-mail address: palle-jorgensen@uiowa.edu 